\title{
Global Optimization Advances in Mixed-Integer Nonlinear Programming, MINLP, and Constrained Derivative-Free Optimization, CDFO
}

\author{
Fani Boukouvala $^{\mathrm{a}, \mathrm{b}}$, Ruth Misener ${ }^{\mathrm{c}}$, Christodoulos A. Floudas ${ }^{\mathrm{a}, \mathrm{b}, *}$ \\ ${ }^{a}$ Texas A\&M Energy Institute \\ Texas A\&M University \\ 302 Williams Administration Building, College Station, TX 77843-1372; USA \\ ${ }^{b}$ Artie McFerrin Department of Chemical Engineering \\ Texas A\&M University \\ College Station, TX 77843-3122; USA \\ ${ }^{c}$ Department of Computing \\ Imperial College London \\ South Kensington SW7 2AZ; UK
}

\begin{abstract}
This manuscript reviews recent advances in deterministic global optimization for Mixed-Integer Nonlinear Programming (MINLP), as well as Constrained DerivativeFree Optimization (CDFO). This work provides a comprehensive and detailed literature review in terms of significant theoretical contributions, algorithmic developments, software implementations and applications for both MINLP and CDFO. Both research areas have experienced rapid growth, with a common aim to solve a wide range of real-world problems. We show their individual prerequisites, formulations and applicability, but also point out possible points of interaction in problems which contain hybrid characteristics. Finally, an inclusive and complete test suite is provided for both MINLP and CDFO algorithms, which is useful for future benchmarking.
\end{abstract}

Keywords: MINLP, Deterministic Global Optimization, Derivative-Free, Black-Box, Grey-Box, Constraints

\footnotetext{
*Author to whom all correspondence should be addressed:

floudasetamu.edu

Preprint submitted to European Journal of Operational Research

(C) 2015. This manuscript version is made available under the Elsevier user license http://www.elsevier.com/open-access/userlicense/1.0/
}

August 28, 2015 


\section{Introduction}

This review introduces recent advances in the global optimization literature in terms of theoretical advances, applications, algorithms, software and test problems. The two categories discussed, Mixed-Integer Nonlinear Programming (MINLP) and Constrained Derivative-Free Optimization (CDFO) or Constrained Grey/BlackBox Problems, encompass a large portion of existing optimization problems and applications.

Using deterministic global optimization of nonconvex MINLP to solve industriallyrelevant problems is not new; Floudas and Aggarwal (1990), Visweswaran and Floudas (1990), Floudas and Visweswaran (1990) and Floudas and Visweswaran (1993) introduce the first, practically-applicable deterministic global optimization algorithms to solve process networks to global optimality $\approx 25$ years before this article was written. Recent increased activity in: developing algorithms; constructing computational frameworks using these algorithms; designing mathematical models suitable for MINLP has developed deterministic global optimization of MINLP to a point where it is becoming promising for real-world use. In practice, global optimization of MINLP is tractable via many heterogeneous algorithms pieced together into a framework; this review highlights complementary algorithmic components.

CDFO refers to problems for which derivatives of the objective function and/or constraints of the original model are not directly used for obtaining the global optimum. In the absence of mathematical structure and derivative information, it becomes impossible to conquer the capabilities of the MINLP literature in terms of problem sizes and theoretical guarantees. In typical CDFO applications, derivative information is either: (1) available but deceptive; (2) prohibitively expensive; or (3) completely unavailable. Historically, the first significant contributions proposing direct-search and pattern search concepts relying simply on function evaluations were published in the 1960s (Hooke and Jeeves, 1961; Spendley et al., 1962; Nelder and Mead, 1965). At that time, the computational and theoretical developments for numerical finite-differentiating and global optimization were inefficient, while direct-search methods were simple in implementation and had the capability of converging to improved solutions with a competitive computational cost. For a thorough introduction to the direct-search history and theoretical developments, please refer to an earlier review of Kolda et al. (Kolda et al., 2003). More recently, significant advances in mathematical analysis, computer power, automatic differentiation and global optimization theory and algorithms have en- 
abled the optimization of complex and large nonlinear problems with theoretical guarantee. However, there is still high interest in CDFO methods because they are suitable for problems which deterministic global optimization methods are unable to handle due to lack of information, noise, non-smoothness and discontinuities (Kolda et al., 2003; Conn et al., 2009b; Rios and Sahinidis, 2013; Martelli and Amaldi, 2014). In the first textbook on Derivative-Free Optimization Conn et al. (2009b) recognize that optimization without derivatives is one of the very challenging open problems in science and engineering, which has a vast number of potential practical applications.

Although the two literatures apparently reference significantly different applications, we aim to show that MINLP and CDFO methods can benefit significantly from each other, or can be synergistically used to solve hybrid problems containing mixed MINLP and CDFO characteristics. For example, MINLP and CDFO problems have been combined in decomposition algorithms where the lower level problem is formulated as a mixed-integer linear or nonlinear problem while the upper level problem is optimized using a derivative-free approach (Martelli and Amaldi, 2014). Moreover, several theoretical advances from the deterministic constrained optimization literature, such as penalty, barrier functions and filters have been used in recent CDFO algorithms (Audet and Dennis, 2006, 2009; Agarwal and Biegler, 2013). Finally, CDFO methods often rely on surrogate approximation models which are fitted based on input-output data, or merit functions for selecting new samples. Consequently, CDFO methods require the solution of parameter estimation problems and global optimization problems which can be solved globally using deterministic MINLP concepts such as branching and construction of underestimators. In this review we distinguish the theory, capabilities and applications of each category separately in order to span the large set of applications which existing methods can solve. However, we also discuss points of interaction of the two, since we believe this will be a major future direction in the global optimization literature. Tables in this manuscript convey the domains associated with each contribution; the purpose is building a quick-access guide of relevant contributions and their integration into advancing global optimization for both MINLP and CDFO. This manuscript also concentrates heavily on applications; this focus reflects our view that examining both MINLP and CDFO through the lens of applications is especially valuable.

This paper is structured as follows. The problem definitions of MINLP and CDFO and their subclasses are introduced in Section 2. Section 3 considers application domains for both categories, while Section 4 mentions prior reviews, textbooks, 
and edited books. Next, the theoretical advances for global optimization for both MINLP and CDFO are described in Sections 5 and 6, respectively. Section 7 discusses existing and future potential interactions between MINLP and CDFO. The algorithmic advances and software for MINLP and CDFO problems are discussed in Sections 8 and 9, while Section 10 presents a test suite of optimization problems.

\section{Problem Definitions}

\subsection{Mixed-Integer Nonlinear Optimization Definitions}

This section defines MINLP and several important sub-classes. Section 2.1.1 defines the most general class of MINLP; Section 2.1.2 defines quadratic constrained, boolean quadratic and quadratic assignment problems and Section 2.1.3 refers to polynomial and signomial mixed-integer optimization problems.

\subsubsection{MINLP}

MINLP is defined as:

$$
\begin{array}{lcl}
\min _{\boldsymbol{x}} & f_{0}(\boldsymbol{x}) & \\
\text { s.t. } & b_{i}^{\mathrm{LO}} \leq f_{i}(\boldsymbol{x}) \leq b_{i}^{\mathrm{UP}} & \forall i \in \mathscr{M}:=\{1, \ldots, M\} \\
& x_{j}^{\mathrm{LO}} \leq \quad x_{j} \leq x_{j}^{\mathrm{UP}} & \forall j \in \mathscr{N}:=\{1, \ldots, N\} \\
& x_{j} \in \mathbb{Z} & \forall j \in \mathscr{I} \subseteq \mathscr{N}
\end{array}
$$

where $\mathscr{M}, \mathscr{N}$, and $\mathscr{I}$ represent sets of constraints, variables, and discrete variables, respectively. The objective and constraints are functions $f_{i}: \mathbb{R}^{N} \mapsto \mathbb{R} \forall i \in$ $\{0, \ldots, M\}$. Parameters $b_{i}^{\mathrm{LO}} \in \mathbb{R} \cup\{-\infty\}$ and $b_{i}^{\mathrm{UP}} \in \mathbb{R} \cup\{+\infty\}$ bound the set of constraints $\mathscr{M}$; parameters $x_{j}^{\mathrm{LO}} \in \mathbb{R} \cup\{-\infty\}$ and $x_{j}^{\mathrm{UP}} \in \mathbb{R} \cup\{+\infty\}$ bound the set of variables $\mathscr{N}$. We assume that it is possible to infer finite bounds on the variables $\boldsymbol{x}$ and that the image of $f_{i}$ is finite on $\boldsymbol{x}$. Typical expressions for $f_{0}(\boldsymbol{x})$ and $f_{i}(\boldsymbol{x})$ are:

$f_{i}(\boldsymbol{x})=c_{i}+a_{i}^{T} \boldsymbol{x}+\boldsymbol{x}^{T} Q_{i} \boldsymbol{x}+\sum_{s \in \mathscr{S}} c_{s, i} \cdot \prod_{j \in \mathscr{N}} x_{j}^{p_{s, i, j}}+\sum_{j \in \mathscr{N}} c_{e, i, j} e^{x_{j}}+\sum_{j \in \mathscr{N}} c_{\ell, i, j} \log x_{j}$

where the powers $p_{s, i, j}$ and coefficients $c_{i}, a_{i}, Q_{i}, c_{s, i}, c_{e, i, j}, c_{\ell, i, j}$ are constant reals; $s \in \mathscr{S}$ indexes the signomial terms. More examples of nonlinear terms found 
in MINLP solver software are listed in Table 1.

Because composite functions may yield combinatorial possibilities beyond the ones listed in Table 1, handling MINLP requires methodologies that effectively address generic, multivariate terms (Floudas, 2000). Complementary techniques for addressing generic functional forms include (1) the $\alpha \mathrm{BB}$ methodology that generates convexifying quadratic or exponential relaxations on expression aggregates via an interval Hessian matrix (Liu and Floudas, 1993; Maranas and Floudas, 1995; Adjiman et al., 1998b,a) and (2) factorable programming trees that break expressions into their component parts through directed acyclic graph representations (McCormick, 1976; Smith and Pantelides, 1999; Tawarmalani and Sahinidis, 2005; Belotti et al., 2009; Mitsos et al., 2009; Vigerske, 2012). Because the methodological tradeoffs between $\alpha \mathrm{BB}$ and factorable programming trees produce complementary convergence behavior (Bompadre and Mitsos, 2011), the most generic global optimization tools are hybrid algorithms that opportunistically exploit the tightest relaxation at each node of a global optimization search tree (Gatzke et al., 2002; Misener and Floudas, 2014a).

\subsubsection{MIQCQP, MIQCP, QAP, Box-Constrained MIQP}

A mixed-integer quadratically-constrained quadratic program (MIQCQP) is defined as a MINLP where all of the nonlinearities are quadratic; symbols have the same meaning as in Section 2.1.1 and equivalent bounded-ness requirements hold:

$$
\begin{array}{lll}
\min _{\boldsymbol{x}} & \multicolumn{1}{c}{c_{0}+a_{0}^{T} \boldsymbol{x}+\boldsymbol{x}^{T} Q_{0} \boldsymbol{x}} & \\
\text { s.t. } & b_{i}^{\mathrm{LO}} \leq c_{i}+a_{i}^{T} \boldsymbol{x}+\boldsymbol{x}^{T} Q_{i} \boldsymbol{x} \leq b_{i}^{\mathrm{UP}} & \forall i \in \mathscr{M}:=\{1, \ldots, M\} \\
& x_{j}^{\mathrm{LO}} \leq x_{j} \leq x_{j}^{\mathrm{UP}} & \forall j \in \mathscr{N}:=\{1, \ldots, N\} \\
& x_{j} \in \mathbb{Z} & \forall j \in \mathscr{I} \subseteq \mathscr{N}
\end{array}
$$

Recall that MIQCQP is mathematically equivalent to a mixed-integer quadraticallyconstrained program (MIQCP) with the introduction of a variable $x_{N+1}$ as the objective function and the addition of a constraint $x_{N+1} \geq c_{0}+a_{0}^{T} \boldsymbol{x}+\boldsymbol{x}^{T} Q_{0} \boldsymbol{x}$.

When $Q_{i}=0 \forall i \in \mathscr{M}$, MIQCQP is referred to as a mixed-integer quadratic program (MIQP). When $\mathscr{M}=\varnothing, \mathrm{MIQCQP}$ is a box-constrained MIQP. Mixedinteger is dropped from all previous definitions whenever $\mathscr{I}=\varnothing$. A boolean quadratic program (BQP) has $\mathscr{M}=\varnothing, \mathscr{I}=\mathscr{N}$, and $x_{j}^{\mathrm{UP}}=1 \forall j \in \mathscr{N}$. 
Many practically-relevant models have some sort of interesting special structure with respect to the matrices $Q_{i}$. For example, the quadratic assignment problem (QAP) is a BQP with problem structure based on multiplying flow and distance matrices (Anstreicher, 2003; Loiola et al., 2007). The max-cut problem, which maximizes the weights on the edges in an undirected graph (Rendl et al., 2010), and the maximum clique problem (Bomze et al., 1999) are other classic problems that may be formulated as MIQP with special mathematical structure enabling solution of large-scale instances. One common way of representing MIQCQP is via an undirected graph representation; see in Figure 1 some of the special structure patterns formed by MIQCQP including process networks, computational geometry, and MIQP (Misener et al., 2014b).

\subsubsection{Mixed-Integer Polynomial and Mixed-Integer Signomial Optimization}

Another important, restricted subclass of MINLP is mixed-integer signomial optimization (MISO); symbols have the same meaning as in Section 2.1.1 and equivalent bounded-ness requirements hold:

$$
\begin{array}{lll}
\min _{x} & \sum_{s=1}^{S_{0}} c_{s, 0} \cdot \prod_{j \in \mathscr{N}} x_{j}^{p_{s, 0, j}} & \\
\text { s.t. } & b_{i}^{\mathrm{LO}} \leq \sum_{s \in \mathscr{S}} c_{s, i} \cdot \prod_{j \in \mathscr{N}} x_{j}^{p_{s, i, j}} \leq b_{i}^{\mathrm{UP}} & \forall i \in \mathscr{M}:=\{1, \ldots, M\} \\
& x_{j}^{\mathrm{LO}} \leq x_{j} \leq x_{j}^{\mathrm{UP}} & \forall j \in \mathscr{N}:=\{1, \ldots, N\} \\
& x_{j} \in \mathbb{Z} & \forall j \in \mathscr{I} \subseteq \mathscr{N}
\end{array}
$$

When $p_{s, i, j} \in \mathbb{Z} \forall s \in \mathscr{S} ; i \in \mathscr{M} ; j \in \mathscr{N}$, the MISO is a mixed-integer polynomial optimization problem.

\subsection{Derivative-Free Optimization Model Definitions}

\subsubsection{Box-Constrained Derivative-Free Models}

The majority of the existing CDFO methods have been developed for box constrained problems, where a set of input variables with known lower and upper bounds affect the objective through a black/grey-box model (BCDFO). 
BCDFO is defined as:

$$
\begin{array}{lll}
\min _{\boldsymbol{x}} & f_{0}(\boldsymbol{x}) & \\
\text { s.t. } & x_{j}^{\mathrm{LO}} \leq x_{j} \leq x_{j}^{\mathrm{UP}} & \forall j \in \mathscr{N}:=\{1, \ldots, N\} \\
& x_{j} \in \mathbb{Z} & \forall j \in \mathscr{I} \subseteq \mathscr{N}
\end{array}
$$

where $\mathscr{N}$ and $\mathscr{I}$ represent sets of variables and discrete variables, respectively, and parameters $x_{j}^{\mathrm{LO}} \in \mathbb{R} \cup\{-\infty\}$ and $x_{j}^{\mathrm{UP}} \in \mathbb{R} \cup\{+\infty\}$ bound the set of variables $\mathscr{N}$.

In BCDFO problems there is only one unknown function (objective $f_{0}(\boldsymbol{x})$ ) which must be either approximated or sampled in order to identify the global optimum within the region bounded by $x_{j}^{\mathrm{LO}}$ and $x_{j}^{\mathrm{UP}}$. We prefer to omit the term Mixed Integer from the formulations of derivative-free problems since the majority of established methods refers to problems for which $\mathscr{I}=\varnothing$. However, noteworthy recent developments incorporating discrete variables will be described in the following sections.

\subsubsection{Nonlinear Constrained Derivative-Free Models}

The general case of CDFO is:

$$
\begin{array}{lll}
\min _{\boldsymbol{x}} & f_{0}(\boldsymbol{x}) & \\
\text { s.t. } & b_{k}^{\mathrm{LO}} \leq f_{k}(\boldsymbol{x}) \leq b_{k}^{\mathrm{UP}} & \forall k \in \mathscr{K}:=\{1, \ldots, K\} \\
& b_{i}^{\mathrm{LO}} \leq f_{i}(\boldsymbol{x}) \leq b_{i}^{\mathrm{UP}} & \forall i \in \mathscr{M}:=\{K+1, \ldots, M+K\} \\
& x_{j}^{\mathrm{LO}} \leq x_{j} \leq x_{j}^{\mathrm{UP}} & \forall j \in \mathscr{N}:=\{1, \ldots, N\} \\
& x_{j} \in \mathbb{Z} & \forall j \in \mathscr{I} \subseteq \mathscr{N}
\end{array}
$$

where $\mathscr{K}, \mathscr{M}, \mathscr{N} \mathscr{I}$ represent sets of known constraints, unknown constraints, variables, and discrete variables, respectively. The known constraints are functions $f_{k}: \mathbb{R}^{N} \mapsto \mathbb{R} \forall i \in\{1, \ldots, K\}$. However, no assumptions can be made about the form of the objective $f_{0}$ and unknown constraints $f_{i}$. The total number of constraints is $\mathscr{K}+\mathscr{M}$, while a general CDFO problem can contain only known constraints, $\mathscr{M}=\varnothing$, or only unknown constraints, $\mathscr{K}=\varnothing$. Parameters $b_{k}^{\mathrm{LO}} \in$ $\mathbb{R} \cup\{-\infty\}, b_{k}^{\mathrm{UP}} \in \mathbb{R} \cup\{+\infty\}, b_{i}^{\mathrm{LO}} \in \mathbb{R} \cup\{-\infty\}$ and $b_{i}^{\mathrm{UP}} \in \mathbb{R} \cup\{+\infty\}$ bound the set of known and unknown constraints $\mathscr{K}$ and $\mathscr{M}$; parameters $x_{j}^{\mathrm{LO}} \in \mathbb{R} \cup\{-\infty\}$ and 
$x_{j}^{\mathrm{UP}} \in \mathbb{R} \cup\{+\infty\}$ bound the set of variables $\mathscr{N}$. There have been several extensions of algorithmic developments for incorporation of general constraints, and fewer developments which deal with discrete variables in general CDFO problems. These will be described in detail in the following sections.

By definition, CDFO literature deals with unknown forms, thus the distinction between different types of formulations is not possible. Theoretical developments in the CDFO field have to- directly or indirectly- rely on function evaluations. A direct approach is purely driven by samples which guide the progress of optimization towards descent directions, while an indirect approach uses samples to parametrize postulated functions which are then optimized to guide the search. For the direct approach, mathematical analysis has enabled to establish theoretical developments which guarantee convergence to local first-order or second-order stationary points using samples (zeroth order information) (Conn et al., 2009b; Kolda et al., 2003). For the indirect case, any theoretical development from the MINLP literature is applicable, since the postulated functions (also known as surrogate functions) should be optimized. These typically contain constant, linear, bilinear, quadratic, exponential, polynomial and even composite terms (Table 1). The dependence on sampling and the complete or partial lack of theoretical closed-form equations makes convergence to global optimality, in the sense which is described in the MINLP literature, extremely challenging and in most cases extremely challenging and in most cases not possible. Thus, it should be stressed that when a problem can be sufficiently described by a well-posed, explicit MINLP formulation, then CDFO methods are not recommended. Despite the above, the applicability of CDFO methods is still significant and we aim to prove this through the next sections of this paper.

\section{Application Domains}

\subsection{MINLP}

Deterministic global optimization for MINLP is highly relevant to application domains where there is high reward for fractional improvements and sufficient time to explore the search space. Table 2 lists some of these domains and gives example problems. Belotti et al. (2013b) also provide list of MINLP applications. The shorter list of Belotti et al. (2013b) incorporates: MIP formulations, local techniques, and convex nonlinearities whereas each of the entries in Table 2 correspond to NLP and MINLP with nonconvex nonlinearities that have been solved using deterministic global optimization. We include a range of references for each 
application to demonstrate the depth of study in some application areas.

The most established application domain is the area of process systems engineering; here the traditional applications are in areas such as refinery operations, water networks, and process synthesis via superstructure optimization. Other domains have emerged in areas such as: biological and biomedical engineering; computational chemistry; computational geometry; finance. A major challenge of globally optimizing MINLP models in both biological engineering and finance is the size of the optimization problems. In our view, it is absolutely critical to examine deterministic global optimization of MINLP through the lens of applications, since deterministic global optimization is important for particular problem domains. Therefore, we are particularly interested in highlighting MINLP approaches applied to practically-relevant problems.

\section{2. $C D F O$}

A primary driving force for the increasing need for CDFO is the development of high-fidelity simulations to represent industrial processes with a high level of detail, coupling multiple physical, chemical, and mechanical phenomena (Forrester et al., 2008). For example, Computational Fluid Dynamic (CFD) models have been used to accurately represent industrial processes, such as gasifiers, turbine combustors, stirred tank reactors and many more. Each simulation of a CFD model for a specific set of input conditions may take several minutes or more, based on the level of detail in the model equations, the geometry complexity and discretization of elements. Computationally expensive models cannot be optimized using deterministic global optimization algorithms which would require multiple function calls to approximate derivative information. Another main advantage of CDFO methods is their independence from derivatives which are highly affected by numerical noise embedded in the original model. Finitedifferentiating techniques are known to fail in the presence of noise, while CDFO methods have been proven to be significantly more consistent in the presence of noise (Conn et al., 2009b; Kolda et al., 2003). An example of this problem arises in cases of expensive large-scale flowsheet simulations with recycle loops, where numerical integration issues lead to unreliable derivatives. In addition, potential applications of CDFO methods are not limited to expensive or noisy computer models, since they can be used to optimize non-smooth problems, with multiple local optima and discontinuities, which are qualities that may cause certain derivative-based methods to fail. Finally, CDFO methods enable the optimization of grey/black-box models which for various reasons such as proprietary restric- 
tions or legacy related issues are available only in the form of input-output data. We define as a black-box system, any simulation for which the user has the ability to change the input variables and obtain the set of output variable values without knowing the underlying model equations which lead to the result. The term Greybox is used for similar problems, with the difference that we do have access to a subset of the model equations.

Typical applications of problems with the above characteristics come from the mechanical, aerospace, civil, chemical and biomedical engineering fields as well as geosciences, where large systems of partial differential equations coupled with nonlinear correlations are formed to represent systems including design aspects, kinetics, fluid dynamics, mass and heat transfer and many more. CDFO methods have a wide range of applications that include aircraft routing, design of hybrid fuel cell vehicles, optimization of cardiovascular geometries, structure design of nano materials, circuit design, flowsheet optimization, process synthesis of biodiesel production and supply chain management. A list of the noteworthy and diverse applications, along classifications related to the type of CDFO problem solved in each work are reported in Table 3.

\section{Major Reviews, Textbooks and Edited Books}

\subsection{MINLP}

A number of major reviews, textbooks, and edited books have been published in recent years; these are organized in Table 4 with brief descriptions. Many of these works address: (1) application domains for MINLP; (2) algorithms for solving MINLP; and (3) software for MINLP.

\section{2. $C D F O$}

Due to the very recent advances in derivative-free optimization theory and the maturity of the literature, there is sparsity of major review papers and textbooks. The first textbooks were introduced in 2008-2009, and several noteworthy review papers have been written in the recent years (Table 5). In Conn et al. (2009b), the authors provide a very comprehensive description of trust-region frameworks, local-search and pattern search, positive spanning sets, sampling, linear and nonlinear local models, interpolation, regression, well-poisedness, simplicial search and more. The focus of this book is unconstrained or box-constrained derivativefree optimization using local-search approaches. The book of Forrester et al. (2008) focuses on model-based search, a different class of CDFO. The authors 
provide theory and algorithms for surrogate model fitting and search criteria for global derivative-free search, from a more application-driven perspective.

\section{Global Optimization Advances in MINLP}

\subsection{Introduction to a Branch-and-Cut Framework}

A crucial component for an effective branch-and-cut framework is algorithm heterogeneity; we need to combine the constitutive elements of: preprocessing, convex underestimators, cutting planes, branching, bounding, and heuristics into an integrated approach. Here we advocate an all-of-the-above approach; an ideal implementation incorporates many possible effective strategies and then selects appropriate algorithms for any given problem.

To organize the individual contributions, Sections $5.2-5.6$ describe algorithmic contributions and Tables $6-8$ present the contributions by area. Figure 2 illustrates the domain of the contributions in the context of a global optimization branch-and-cut framework.

\subsection{Reformulations and Other Preprocessing Methods}

Automatic reformulations, while common in MIP, are relatively rare in MINLP (Bussieck and Vigerske, 2010). Recommendations made to modelers (i.e., nonautomatic reformulations) often include: using linear formulations if possible (Harjunkoski et al., 1996); using convex functional forms if possible (Harjunkoski et al., 1998); dissaggregating terms (Tawarmalani et al., 2002; Tawarmalani and Sahinidis, 2002b); adding redundant constraints into the model formulation (Karuppiah and Grossmann, 2006; Ahmetović and Grossmann, 2011; Ruiz and Grossmann, 2011b). Any MINLP solution method introducing auxiliary variables is doing automatic reformulations, but it is currently unclear (for the general case) what are the best automatic reformulations.

Some semi-automatic and fully-automatic reformulations have been developed for MIQCQP; these often take the form of reducing the number of nonconvex bilinear terms. Ben-Tal et al. (1994) showed that the dual of MIQCQP is sometimes smaller than the primal, Audet et al. (2004) eliminated bilinear terms in the pooling problem through mass balances at the intermediate nodes, and Liberti and Pantelides (2006) generalized the contribution of Audet et al. (2004) to automatically eliminate unnecessary bilinear terms in MIQCQP.

A stand-alone reformulation engine with no associated solver is ROSE, the reformulation optimization software engine (Liberti et al., 2009a, 2010). The MIQCQP 
solver GloMIQO (Misener and Floudas, 2013) represents a major effort to elucidate special structure via reformulations and integrates reformulation techniques that can be implemented generically and applied universally. The GloMIQO reformulation uses the observation that disaggregating bilinear terms tightens the relaxation of MIQCQP and actively takes advantage of any redundant linear constraints added to the model; GloMIQO may add bilinear terms to the model formulation to create tight reformulation-linearization technique relaxations.

Other preprocessing algorithms include: heuristics for developing a good upper bound (see Section 5.6); detecting symmetry (Liberti, 2012); building a directed acyclic graph representation of the MINLP (Belotti et al., 2009; Vigerske, 2012).

\subsection{Tight Convex Underestimators}

Nonconvex terms in MINLP are typically replaced with rigorous convex underestimators for solving the lower-bounding problem of the MINLP; solving the relaxed, convex minimization problem gives deterministic lower bounds on the global optimum (Floudas, 2000). In the context of a branch-and-bound deterministic global optimization algorithm, sufficient convergence conditions are (Horst and Tuy, 1996): (1) any unfathomed node can be further refined and (2) the original MINLP and underestimating problem converge as the refinements approach infinity.

Table 6 records a variety of rigorous convex underestimators designed for nonconvex MINLP. Observe that this part of the MINLP framework has been richly studied; deriving tight convex underestimators for MINLP is an especially important component of solving MINLP. There is a trend towards developing good underestimators for very specific terms, for example: Meyer and Floudas (2003, 2004) introduce the explicit facets of the convex and concave envelope of trilinear terms; Liberti and Pantelides (2003) find the convex hull for odd degree monomials; Gounaris and Floudas (2008a) give results on specific products of univariate functions. The solver ANTIGONE (Misener and Floudas, 2014a) implements these heterogeneous choices using a polymorphic programming strategy; specialized terms inherit from more general base classes of terms.

With respect to implementations, a dominant trend is in hybrid approaches that implement multiple possible relaxations and have them compete in real-time while solving an MINLP (Gatzke et al., 2002; Bompadre and Mitsos, 2011); variants of this heterogenous strategy are implemented in ANTIGONE (Misener and Floudas, 2014b,a) and BARON (Zorn and Sahinidis, 2014a). A trend complementing the current dominance of hybrid relaxations is the area of reducing relaxations to their 
necessary set. As the possible number of relaxations grow, several authors suggest eliminating weaker cuts based on logical inferences or choosing appropriate relaxations based on extensive computational experience: Lundell et al. (2009); Sherali et al. (2012); Cafieri et al. (2012); Ballerstein and Michaels (2014); Dalkiran and Sherali (2013); Skjäl and Westerlund (2014); Guzman et al. (2014).

\subsubsection{Statically-Generated Underestimators}

Convex underestimators may be generated statically at each node (i.e., in branchand-bound) or at each iterative refinement level. In a branch-and-cut framework, tight convex underestimators may expedite deterministic global optimization by increasing the objective lower bound with respect to divide-and-conquer algorithms based on interval analysis (Adjiman et al., 1998a,b). Ideally, convex relaxations both tightly approximate the original function and are computationally inexpensive to generate, but these design goals are often at odds with one another. The convex hull, the smallest convex set containing the original nonconvex term or function, is the tightest possible convex underestimator.

Tight convex underestimators are also valuable in adaptive refinement approaches that continue refining the root node rather than spatially branching on the search space. The solvers $\alpha \mathrm{R}$ and $\alpha \mathrm{SGO}$ solve a sequence of convex MINLP with progressively more integer break points (Lundell et al., 2013). Iterative refinement of tight underestimators has also been applied to examples including gas networks (Domschke et al., 2011; Geissler et al., 2013).

\subsubsection{Cutting Planes}

In addition to statically-generated underestimators, convex underestimators may be added dynamically as cutting planes where, after a node is optimized, separating hyperplanes are added to the underestimating optimization problem and the node is resolved (usually via a warm start). By only adding constraints which are violated in the solution of the underestimated problem, optimization algorithms can avoid introducing more constraints than necessary; this is especially relevant in polyhedral relaxations of many-dimensional expressions where the number of candidate facets may grow combinatorially (Misener et al., 2014b).

Cutting planes developed for MINLP include those based on: pseudo-convex MINLP problems (Westerlund et al., 1998), outer approximation of convex terms and linearization of other convex underestimators (Tawarmalani and Sahinidis, 2005; Misener and Floudas, 2014b), multi-term quadratic expressions (Bao et al., 2009; Luedtke et al., 2012; Misener et al., 2014b), multilinear functions (Rikun, 
1997; Belotti et al., 2010b; Qualizza et al., 2012), optimizing convex quadratic functions over nonconvex sets (Bienstock and Michalka, 2014), and other cutting plane classes based on nonlinear functional forms (D'Ambrosio et al., 2010; Richard and Tawarmalani, 2010). A review on cutting plane methods for MINLP can be found in Nowak (2005); multivariable and multiterm relaxations are typically favoured for MINLP because the tightest convex relaxation of each individual is not typically equivalent to the tightest possible relaxation of the entire MINLP (Westerlund et al., 2011).

\subsection{Domain Branching}

Dividing the search space via domain branching approaches manageable problem sizes via recursion. Most implementations divide one node into two by branching on a single variable (Adjiman et al., 1998a,b; Belotti et al., 2013b); the commonlyused approach is reliability branching, a technique that integrates strong branching with a pseudocost heuristic to predict the best branching variable (Achterberg et al., 2005; Belotti et al., 2009). Applying reliability branching to MINLP was introduced by Belotti et al. (2009) for the solver software implementation Couenne; it is additionally implemented in ANTIGONE / GloMIQO (Misener and Floudas, 2012b, 2013, 2014a) and SCIP (Berthold et al., 2012b,a). Table 7 records an interesting array of branching methods designed for MINLP.

\subsection{Feasibility- and Optimality-Based Bounds Tightening}

Implementations for solving MINLP models often rely heavily on bounds tightening to reduce the feasible space. Feasibility-based bounds tightening (FBBT), which uses interval arithmetic to place bounds on expressions by recursively overestimating each of the participating functions and operators, is the simplest and most computationally inexpensive technique (Adjiman et al., 1998a,b; Androulakis et al., 1995; Audet et al., 2000; Belotti et al., 2009; Neumaier, 1990; Sherali and Tuncbilek, 1995, 1997; Wolfe, 2000). An alternative, optimization-based bounds tightening (OBBT) methodology for determining these interval estimates is to minimize and maximize the expression under the bound constraints and possibly additional linear and convex constraints from the problem. These subproblems provide tighter bounds than interval arithmetic (Maranas and Floudas, 1995; Tawarmalani and Sahinidis, 2002b). A decreased number of nodes in the branchand-bound tree may justify the increased computational effort needed to find better estimates. 
FBBT and OBBT represent extremes. FBBT is cheap but not especially effective; OBBT is computationally demanding but may significantly reduce the bounds. Table 8 organizes contributions to domain reduction algorithms.

\subsection{Heuristics}

The final piece of any successful deterministic global optimization framework is good heuristic algorithms. While local MINLP methods are possibly applicable to deterministic global optimization, it is important to understand that effective heuristics for a deterministic MINLP framework are not necessarily the same as effective stand-alone heuristics. So far there are relatively few works that consider heuristics in the broader context of deterministic global optimization; exceptions include the protein structure prediction algorithm of Klepeis et al. (2003a,b), Undercover (Berthold and Gleixner, 2013a), and the computational studies of Berthold (2014).

There are several heuristic algorithms recently developed for finding a first feasible solution of MINLP; a few of the promising feasibility pumps include those of: Bonami et al. (2008); Liberti et al. (2011); Boland et al. (2012); D’Ambrosio et al. (2012); Nannicini and Belotti (2012). Fast-fail algorithms have been identified as the most effective for integration into deterministic global optimization of MINLP (Berthold, 2014). There are many more heuristic MINLP algorithms (Belotti et al., 2013b), but their applicability with respect to deterministic global optimization has not been established (Berthold, 2014).

\subsection{Numerical Issues}

Numerical issues represent an interesting trade-off with respect to correctness versus solving speed. While some work has been done in rigorous global optimization that formally verifies nonlinear functions including semialgebraic and transcendental functions (Domes, 2009; Domes and Neumaier, 2014), the most commonly-used solver software generates relaxations and cutting planes via floating point arithmetic and then uses floating point-based LP and NLP solvers for finding underestimators and heuristic solutions. Numerical instability can be at least partially mitigated using validated interval arithmetic (Brönnimann et al., 2003, 2006; de Moura and Passmore, 2013) for FBBT but, especially for badlyscaled optimization problems, combining diverent solving strategies may induce numerical trouble because of the variance in tolerances between solvers (including clash between different sub-solvers of a single meta-solver). 
Another interesting set of numerical issues related to global optimization comes from a solver trying to meet user-set tolerances; the cluster problem refers to relaxations slowly converging to a global solution by generating many boxes around a single minimizer (Du and Kearfott, 1994; Wechsung et al., 2014). Mitigating the cluster problem typically involves minding relaxation convergence rate (Bompadre and Mitsos, 2011).

\section{Global Optimization Advances in CDFO}

Undoubtedly, lack of derivative-information is a very important critical loss for efficient and fast optimization. In the area of Constrained Derivative-Free Optimization, CDFO, recent developments either rely on input-output data (direct-search), or employ an intermediate step of developing cheaper and smooth approximate models using the input-output data (model-based). Additionally, CDFO methods can be divided into two categories based on their search strategy: (1) local-search methods which require either a single initial point or initial local trust region and proceed making small movements within a local subspace, and (2) global search methods which explore the entire bounded region during each iteration. The third main categorization of black-box methods is related to the nature of the search strategy, which can be deterministic or stochastic. Finally, there have been recent developments of hybrid approaches which combine characteristics from different algorithmic developments.

\subsection{Local Search Methods}

\subsubsection{Direct search methods}

In this category, the theoretical developments focus on sampling using positive spanning sets, assurance of a descent mechanism. Convergence to first-order and second-order optimal solutions is guaranteed under certain smoothness and differentiability assumptions of the objective function and the constraints within the local region (Kolda et al., 2003; Conn et al., 2009a). Sampling-based local CDFO optimization includes direct-search, pattern-search and simplex based methods. Convergence has been studied extensively in the literature, where a set of updating rules of the trust region guarantee convergence to local optima when the size of the trust-region is sufficiently small. All local direct-search CDFO methods incorporate a set of rules to ensure descent away from stationarity, which in concept is identical to the mechanism followed by derivative-based algorithms. Direct-search methods require that the function is sampled at locations defined 
by positive spanning sets in order to move towards the direction of the best function value. Moreover, the mechanism of local CDFO methods must ensure that strict criteria of the geometry of the sampling design are satisfied, in order to theoretically guarantee true stationarity. Measures of geometry such as the cosine measure of positive spanning sets, the normalized simplex volume and the a-poisedness constant are described in detail in (Conn et al., 2009b). Finally, local CDFO algorithms converge when the step size, mesh size, simplex diameter or line-search parameter is sufficiently small, which has been theoretically linked with convergence to stationary points. A schematic of local-search type steps is shown in Figure 3, where initially a random initial point is selected, followed by a set of expansions and contractions until the step size is driven to zero. It should be noted that purely direct-search methods do not exactly follow the schematic representation shown in Figure 3, since in the direct-search case the new centers must lie on a predefined lattice. Figure 3 is developed as a general representation of both direct-search and trust-region search which will be described next. The disadvantages of local direct-search CDFO are the high dependence on the initial point, entrapment within closest local optimum, and large number of function calls to guarantee convergence (Audet et al., 2008b; Conn et al., 2009b). In order to increase the probability for convergence to the global optimum, multi-start approaches are proposed, which are not efficient in cases where the model of interest is computationally expensive. However, when a good starting point is available or when sampling does not require significant computational cost, CDFO methods in this category guarantee convergence to stationarity with reliability.

Algorithmic developments and convergence have been studied for box-constrained problems (BCDFO) (Lewis and Torczon, 1999; Lucidi and Sciandrone, 2002; Audet and Dennis, 2002; Garcia-Palomares et al., 2013); for known linear constrained problems (Lewis and Torczon, 2000; Lewis et al., 2007; Audet and Dennis, 2002; Kolda et al., 2006); for general constrained problems (Abramson, 2002; Abramson and Audet, 2006; Abramson et al., 2009c; Audet and Dennis, 2004, 2006; Audet et al., 2008b; Audet and Dennis, 2009; Liuzzi et al., 2010; Audet et al., 2010). Constraints are handled by penalty methods such as extreme barrier (Abramson and Audet, 2006; Abramson et al., 2009c; Audet and Dennis, 2006; Audet et al., 2008b), progressive barrier (Audet and Dennis, 2009), filters (Audet and Dennis, 2004), sequential penalty merit functions (Liuzzi et al., 2010); smoothed exact $l-\infty$ penalty function (Liuzzi and Lucidi, 2009) and exact penalty merit functions (Fasano et al., 2014; Gratton and Vicente, 2014), or restoration steps which are independent from objective function (Martinez and Sobral, 2013; 
Arouxét et al., 2015). Allowing pattern-search methods to handle a dense set of polling directions instead of a finite set of polling directions was a significant development for studying the convergence of generally constrained derivative-free problems which are Lipschitz even near a limit point (Audet and Dennis, 2006; Audet et al., 2008b). A recent methodology proposed in (Vicente and Custódio, 2012) studies convergence of local-search adaptive search in the case of discontinuous objective functions and general constraints, which is useful for many real applications. Methods which are based on Augmented Lagrangian concepts for treatment of constraints have the disadvantage of a dependence on the selected parameters (or weights) of the penalty terms. However, such methods provide better theoretical guarantees to locally optimal solutions, when compared to filter based methods.

Local-search CDFO contributions have been extended for handling mixed-integer variable problems in the work of (1) Audet and Dennis (2001) for box-constrained problems; (2) Abramson et al. (2001) for general constrained CDFO problems through a filter approach; (3) Lucidi et al. (2005) and Abramson et al. (2009a) for constrained problems with known linear constraints; (4) Liuzzi et al. (2012) for box-constrained problems based on the earlier method desribed in (Lucidi and Sciandrone, 2002); (5) Liuzzi et al. (2015b) for general constrained mixed-integer problems extending an earlier sequential penalty approach described in (Liuzzi et al., 2010). A contribution which diverges from the above, but belongs in the local-search direct-search category is developed for purely discrete problems, for which an implicit and dense closed set is available (Vicente, 2009). The main challenge for extending the capabilities of local-search derivative-free methods to handle discrete variables is the need to assume a user-defined criterion for local optimality with respect to the discrete variables. User-defined discrete local optimality requires setting the set of neighbors amongst which the local optimizer is identified. Convergence to stationary points is guaranteed using similar concepts to the continuous case, however, the local mesh of points is an augmented set containing positive spanning directions defined by combinations of discrete and continuous variables within the local trust region; or else a union of a finite number of lattices in the continuous space with the integer space (CDFO).

\subsubsection{Model-based methods}

Local search model-based methods are also described as trust-region methods. Instead of relying simply on function evaluations, a trust-region subproblem is first defined by fully linear/quadratic models, typically built from polynomial inter- 
polation and regression, or other interpolating models such as kriging and radial basis functions. Similar to direct-search CDFO, descent is guaranteed away from stationarity by combining contraction and expansion mechanisms with the final objective of the reduction of the trust-region radius. As opposed to direct-search, the next iterate center point does not need to lie on the pattern, mesh or simplex edges (Figure 3). Algorithmic comparisons have shown that by using a local model which approximates the true function with acceptable accuracy within the trust region, convergence is expedited (Conn et al., 2009b).

Several noteworthy box-constrained trust-region CDFO methods have been developed such as the Surrogate Management Framework of Booker et al. (1999) and the extension of mesh adaptive search in (Abramson et al., 2012), which both use kriging models; Oeuvray (2005); Oeuvray and Bierlaire (2007) which use radial-basis function models (BOOSTERS algorithm); the Implicit-filtering algorithm which uses fully quadratic approximations of the gradient in order to move towards descent directions along negative simplex gradients (Gilmore and Kelley, 1995); the BOBYQA algorithm in (Powell, 2009) which uses local quadratic models; and finally a recent approach which uses interpolating polynomials (Gratton et al., 2011). The LINCOA algorithm (Powell, 2013b) uses quadratic approximations for the unknown objective function and performs optimization in the presence of known linear constraints. Conejo et al. (2013) develop a method which uses local quadratic models of the objective function for problems with a known convex feasible region. General constrained CDFO problems have been studied by Caballero and Grossmann (2008) and Sankaran et al. (2010) using local kriging models for the objective and constraints; Powell (1994, 2013a) developped the COBYLA algorithm which uses local linear approximations for the unknown objective and the unknown constraints which is an approach revisited recently by March and Willcox (2012); Conn and Le Digabel (2013) employ quadratic models for the objective and constraints; Müller et al. (2013) use radial-basis function models; and finally Müller and Shoemaker (2014) use an ensemble of various surrogate-models to best fit the objective and constraints of the problem. A recent extension of the BOBYQA algorithm for mixed variable programming has been published which uses quadratic approximations and local integer search, with guaranteed identification of locally optimal points (Newby and Ali, 2014). Quadratic models are also used to represent inequality constraints in conjunction with mesh-adaptive direct-search (Audet et al., 2014), in order to expedite local convergence with a fewer number of samples. A representative collection of interpolating and non-interpolating surrogate models used for all model-based methods 
are reported in Table 9.

\subsection{Global search methods}

\subsubsection{Direct search methods}

Global-search CDFO refers to methods which do not require a starting point, while they consider the region defined by $x_{j}^{\mathrm{LO}}$ and $x_{j}^{\mathrm{UP}}$ as the search space of the algorithm. The DIRECT algorithm (Jones et al., 1993) divides the box constrained space into rectangles and samples each center point. Progression of the algorithm occurs by dividing rectangles with lowest values, until typically a maximum number of function calls is reached. Huyer and Neumaier (1999) developed a global optimization methodology for box constrained problems called Multilevel Coordinate Search (MCS), which allows an irregular partitioning procedure, while new points do not need to be center points of the rectangles. Global-search directsearch methods have been developed for box-constrained problems and have been tested on low-dimensional problems. A recent extension of the DIRECT algorithm was proposed in Di Pillo et al. (2013), where the authors employ an exact penalty function and an optional local search extension in order to locate improved solutions to general constrained problems. This method is tested on a large set of problems with up to 10 dimensions and constraints.

\subsubsection{Surrogate model based methods}

Theoretical developments for the methodologies which belong to the global-search model-based group are related to surrogate-model fitting, parameter estimation, sampling design and criteria for selecting the next sampling point. This category of CDFO is particularly suitable for computationally expensive applications. Theoretical convergence can be sacrificed, if good optimal solutions are located with a minimum number of function calls. In the global search CDFO literature, theoretical developments lie mostly in the combinations of surrogate-models used and the criterion for selection of the next promising sample until convergence. An overview of the steps for global model-based CDFO is shown in Figure 4, where an initial set of samples is collected within the entire search space; models are fitted for the objective, constraints, or the lumped penalized objective using parameter estimation; the formulated approximation model is optimized using derivative-based optimization in order to find promising solutions which are added to the sampling set. This procedure is repeated until the convergence criteria of the algorithm (i.e. no improvement in consecutive iterations, maximum number of function calls) are met. As pointed out in Figure 4, there are two stages during 
which CDFO can really benefit from theoretical advances of MINLP, namely parameter estimation and optimization of the fitted model. This is an aspect which has not been discussed in the literature significantly, since typically local solvers are used to optimize the formulated surrogate model which may in turn be nonconvex and exhibit multiplicity of local optima. The majority of the developed algorithms guarantee asymptotic convergence in the limit of infinite samples (Jones, 2001; Forrester et al., 2008; Forrester and Keane, 2009; Jakobsson et al., 2010; Jones et al., 1998; Müller et al., 2013).

The key theoretical difference of all global-search CDFO methods lies in the surrogate method used to approximate the unknown models, as well as the criterion or strategy used to locate the next sampling point. The different types of interpolating and non-interpolating surrogate models used in the literature are summarized in Table 9. The second key theoretical component is the strategy for location of the next sampling location, since the fitted model accuracy during the initial iterations is not reliable in regions which have not been sampled sufficiently. As a result, if the algorithm proceeds by blindly trusting the model predictions, there is high risk that the method will converge prematurely to a local optimum, without exploring promising regions of the search space. Jones et al. (1998) provided a formal mathematical criterion (Expected Improvement, EI) which ensured that both the model prediction as well as the uncertainty of the model are incorporated within one equation during each iteration in order to provide the next sampling point. Using theoretical developments from the MINLP literature, such as convex underestimators and bounds tightening, Jones et al. (1998) optimized the EI Criterion, ensuring that any new sample will have at least some distance from existing samples. The EI criterion based algorithm uses interpolating kriging surrogate models and was developed for box constrained problems. Other global-search surrogate-based methods which employ kriging approximations within box constrained regions have been developed by Forrester and Jones (2008) and Quan et al. (2013). Radial-basis functions have also been used in the global-search surrogate-based CDFO methods for box-constrained problems (Björkman and Holmström, 2000; Holmström et al., 2008b; Jakobsson et al., 2010; Regis and Shoemaker, 2007a,b,c, 2013b). Yao et al. (2014) use a hybrid Neural-Network Radial-basis function model within a global-search framework which forces gradient estimations in local regions to agree with actual gradient information. Jones (2001) compares the performance of kriging-based surrogate models to quadratic non-interpolating models for global-search optimization, while Viana et al. (2013) develop approaches using multiple surrogate predictions to locate promising new 
sampling points within a box-constrained region.

Several contributions have extended the capabilities of the above methods to handle constraints. Le Thi et al. (2012) employ radial basis functions to approximate the black-box objective function and introduce a difference of convex functions (D.C.) programming approach for optimizing the surrogate formulation with box and known linear constraints. Sasena et al. (2002) use probability of constraint satisfaction, extreme barrier methods and treatment of each constraint using individual surrogate functions to handle general non-linear constraints within the EI framework, while Parr et al. (2012) use multi-objective optimization. Villemonteix et al. (2009a,b) introduce a new minimizer entropy kriging-based criterion for locating new samples, which must be evaluated using conditional simulations in order to incorporate constraint satisfaction, while Kleijnen et al. (2010) develop a method for solving constrained Integer Nonlinear Problems using kriging approximations. General constraints have been approximated by radial basis functions in the works of Regis and Shoemaker (2005); Regis (2011, 2014); Rashid et al. (2013), while Holmström et al. (2008b) incorporate constraints in an aggregated penalty term which is added to the objective function, transforming the problem to box-constrained. Boukouvala and Ierapetritou (2013, 2014) employ kriging approximations for the objective function and the lumped feasibility-based criterion which aims to represent the feasible space of the problem through a single function. Recent work of Müller et al. (2013) extends the capabilities of the radial-basis function based black-box method for mixed-integer problems, under the assumption of continuity of the objective and constraints surfaces with respect to the discrete variables.

\subsection{Stochastic methods}

Constrained stochastic CDFOs come from the evolutionary literature and rely on random sampling strategies, or strategies based on probabilistic criteria. Random search initiated as a concept in the 1950's, proving asymptotic convergence results and the literature of Stochastic CDFO methods has been covered in the book of Zabinsky (2003) and the reviews of Das and Suganthan (2011) and Martelli and Amaldi (2014). The first algorithm to use random samples from a centroid template was the COMPLEX method (Box, 1965), which proceeded by replacing the worst identified feasible points. Controlled Random Search algorithms such as Simulated Annealing and Hide and Seek were later on developed, based on which new solutions are generated based on a sequence of probability distributions. Other developments which belong in this category are Genetic Algorithms, 
Particle Swarm Optimization, Memetic Algorithms and Tabu-search (Ong et al., 2003; Das and Suganthan, 2011; Pal et al., 2012; Sun et al., 2013). There exist several developments which extend the capabilities of stochastic methods to mixed-integer optimization, such as the work of Laguna et al. (2014) which is developed to solve purely discrete variable problems with general constraints using scatter search. Overall, it is believed that these methods require a significant number of evaluations and should be used as a resort, in cases where the space is necessarily large, complex, or poorly understood and more sophisticated mathematical analysis is not applicable (Conn et al., 2009b). This review does not contain a comprehensive list of stochastic optimization literature, since this is has been performed earlier.

\subsection{Hybrid methods}

Several algorithmic developments combine two or more techniques in order to exploit the advantages of different methodologies in terms of convergence, sampling requirements and efficiency. Pattern search methods have been combined with genetic algorithms, and the resulting hybrid methodology has significantly better performance when compared to pure evolutionary algorithms (Egea et al., 2007a,b, 2010). Griffin and Kolda (2010) integrate the DIRECT algorithm with a stochastic generating set search approach to solve box-constrained problems, while Hemker and Werner (2011) combine DIRECT with surrogate-based local search for general constrained CDFO problems. More recently, Liuzzi et al. (2015a) propose modifications of the DIRECT algorithm in order to expedite convergence using local searches and transformations of the feasible domain. Vaz and Vicente (2007, 2009) integrate Particle Swarm Optimization (PSO) with pattern search components for solving box constrained and linearly constrained CDFO problems. Martelli and Amaldi (2014) combine three different techniques, constrained Particle Swarm, pattern search and the COMPLEX method to solve nonsmooth problems with multiple nonlinear constraints.

Moreover, decomposition methods have been developed to solve complex problems such as optimization of steam networks (Colmenares and Seider, 1989), where steam pressures and temperatures are optimized in the lower level using deterministic global optimization, while water and mass flow is optimized in the upper level using the COMPLEX stochastic method. Similarly, Gassner and Maréchal (2009) develop a decomposition algorithm for total-site optimization integrated with heat exchangers and utility systems. Following their approach, the upper level is a black-box non-smooth problem, while the lower level problem is formulated as a MINLP. Martelli et al. (2011) follow a similar approach 
to optimize a heat recovery steam cycle application; decomposing the problem into a lower level Linear Program and a Constrained Particle Swarm upper level program. Even though the aforementioned decomposition approaches constitute an interesting approach for combining CDFO with MINLP, the stochasticity of the CDFO methods used for obtaining bounds on the upper level problems cannot guarantee global convergence or uniqueness of results.

In addition, there exist several methodologies using surrogate models to approximate a part of an original MINLP model which is treated as a black-box, and develop an iterative approach collecting additional samples in order to improve convergence towards a global solution (Davis and Ierapetritou, 2007, 2008, 2009; Henao and Maravelias, 2011). Finally, Garcia-Palomares et al. (2006) propose combining global search with a final local search; a recent development combines global search and local direct-search optimization in an box-constrained DFO algorithm (GLODS) which aims to identify multiple local solutions without using random multi-start methods (Custódio and Madeira, 2015).

\section{Connections between CDFO and MINLP}

Undoubtedly, in the CDFO literature each development may have a significance since there are many characteristics which will dictate its relative performance in a specific application (i.e., model computational cost, smoothness, non-linearity, number of constraints, number of dimensions, sparsity, complex and reduced feasible regions and more). Moreover, despite the fact that convergence guarantees are important from a theoretical perspective, the number of function calls is a limiting factor towards attainment of even local optimality in many practical applications. Hence, in this case it is more important to attain better solutions with less number of samples- at the cost of theoretical convergence. Evidently, advances in CDFO have focused in the area of local optimization, while the literature is still far from guaranteeing convergence to global solutions due to the lack of mathematical structure, derivative-information and valid lower and upper bounds. Extending the capabilities of local-search CDFO methods to global methods have been proposed using multistart approaches (Audet et al., 2010), which are suitable only in the case of fast black-box simulations for which multiple function calls would be possible. Designing computationally efficient algorithms with parallelization capabilities is required in this case, (Audet et al., 2008c), however as the simulation time of the optimization model increases sampling issues will still limit the applicability of local-search methods. 
On the other hand, there has been increasing interest in global-search model-based methods which typically employ surrogate approximations to represent unknown or black/grey-box components of a system, while they do not require an initial point and do not restrict the search to a local region. This is an interesting approach which can be seamlessly integrated within existing MINLP software described in Section 5, combining any known information with unknown while taking advantage of all of the existing capabilities of MINLP theory. Using this paradigm, hybrid MINLP/CDFO global optimization solvers can be developed to solve problems for which a large amount of the mathematical structure is known, while a fraction of it is provided as an input-output black-box system. For example, if a large-scale problem can be decomposed into two separable parts as follows: (1) a deterministically known MINLP formulation and (2) a black-box or unknown set of equations, then efficient decomposition strategies have the potential of being developed. In such an example, one can iterate between fixing variables which are involved in the black-box part and obtaining upper and lower bounds on the problem by using a global MINLP solver. Such an approach would then limit the search bounds for the employed CDFO algorithm. However, for such an approach to work efficiently there is more work that needs to be done towards obtaining bounds for the solutions of the CDFO problems within larger and more complex search regions.

Additionally, there still exist challenges in the area of MINLP optimization (e.g. trigonometric functions) for which advances in CDFO such as the development of surrogate approximations with error bounding properties can be developed in order to develop better estimators of the non-convex functions.

Another important future direction of CDFO methods is the incorporation techniques to detect the structure of the problem and the model sparsity in order to reduce the complexity of the problem as well as to reduce the search-space. However, since the analytical forms are not available explicitly, machine learning techniques, sensitivity analysis and statistical methods will play an important role towards this direction. For example, variable ranking methods have been incorporated in recent works (Adjengue et al., 2013; Regis and Shoemaker, 2013a) with promising results for problems with increased dimensionality. Finally, it is certain that CDFO literature has still yet to take full advantage of the theoretical developments of MINLP theory. Existing literature has focused on the use of surrogate model functions which lead to complex and non-convex representations, which in turn are not globally optimized using existing MINLP software. This trend creates several questions: 
1. Can the efficiency of an algorithm be improved when the complex surrogate functions used to represent black-box models are globally optimized?

2. How useful and reliable is a fitted surrogate model in representing an unknown function, when the parameters of the model are not globally optimized? What effect does the uncertainty in the model parameters have in the performance of the method?

3. Is it possible to guarantee global convergence by developing rigorous bounds on the error estimates of fitted models?

Based on all of the above, it is expected that there are still many exciting contributions that will be made in the area of global CDFO, which will be highly intertwined with the MINLP literature.

\section{Global Optimization Algorithms and Software for MINLP Models}

A summarized list of the currently available global optimization solvers for MINLP and their original sources is included in Table 10. Global optimization solver software for MINLP has been covered most completely by Bussieck and Vigerske (2010); the book of Belotti et al. (2013b) and the review of D'Ambrosio and Lodi (2013) also discuss several software implementations for MINLP. We augment their work with Table 11, which documents algorithmic advances for each of several deterministic global optimization codes for MINLP. The emphasis here is on the way each code base also represents an algorithmic framework with many constituent components.

The newest global optimization code bases for MINLP are: $\alpha \mathrm{R} / \alpha \mathrm{SGO}$ (Lundell and Westerlund, 2009, 2012; Lundell et al., 2009, 2013), under active development at Abo Akademi; ANTIGONE (Misener and Floudas, 2014b,a), released in 2013; GloMIQO (Misener and Floudas, 2012b, 2013), released in 2012; SCIP (Vigerske, 2012), MINLP component released in 2012.

The newest code bases successfully implement some of the most recent algorithms: $\alpha$ SGO uses exponential and power-law transformations for signomials (Maranas and Floudas, 1997; Lundell and Westerlund, 2009) and the spline $\alpha \mathrm{BB}$ underestimators of Meyer and Floudas (2005b) for general terms; ANTIGONE couples a hybrid relaxation strategy with polymorphic programming techniques to admit many possible relaxations into the lower bounding problem (Misener and Floudas, 2014a); GloMIQO focuses on finding and exploiting special mathematical structure in MIQCQP (Misener and Floudas, 2014b); SCIP integrates several new relaxations and bounding techniques into the software design (Vigerske, 
2012). Several of the older code bases are also actively maintained with new algorithmic advances: $\alpha \mathrm{BB}$ has been extended to incorporate bilevel optimization (Kleniati and Adjiman, 2014a,b); BARON has been extended to consider intermediate polynomial substructures (Zorn and Sahinidis, 2014b); LINDO has been extended with a range of functional forms (Lin and Schrage, 2009).

\section{Global Optimization Algorithms and Software for CDFO}

In the literature, there exist few methodologies which are designed to handle constraints efficiently, some of which have also lead to commercially available software. Box constrained grey/black-box optimization software has been described in the recent review of Rios and Sahinidis (2013). Table 12 presents the most recent available software which can solve box-constrained CDFO and general constrained CDFO problems, with or without discrete variables. Methods which can handle general non-linear constraints and/or discrete variables are highlighted in the table. In Table 12, we have restricted the references to software that is easily available to the academic community, in most cases at no cost for the user.

\section{Test Suite and Comparative Studies}

\subsection{MINLP}

Table 13 presents an MINLP test suite. This test set ranges in application from computational geometry problems to process networks problems; the case studies have $1-17,136$ nonlinear terms. Misener and Floudas (2014a) show complete results across this entire test set and demonstrate the capacity of each available solver with respect to several classes of criteria.

\section{2. $C D F O$}

The test suite of problems suitable for CDFO problems has not been consistent across the literature due to the wide range of applications and the different nature of the methodologies which are typically developed for the purpose of a specific case study. For example, several algorithms have been developed for computationally expensive applications, for which convergence to stationarity is not of great importance due to strict upper limits in function calls. Such algorithms typically employ global model-based concepts and are tested on low-dimensional problems. On the other hand, local pattern search methods are typically developed for faster grey/black-box models, which may be noisy or non-smooth. Such methods typically require multiple function calls to guarantee convergence to a local 
minimum and are tested on problems with higher dimensions. Consequently, it is difficult to create a pool of benchmark problems for CDFO problems due to their limitations in terms of number of input variables and number of constraints. Table 14 summarizes a comprehensive set of box-constrained and general constrained global optimization set of problems which may potentially be used for evaluation of CDFO developments to test the performance, sampling requirements and dimensionality limits of a new algorithmic development. The majority of the sets of problems are taken from collections in books (Floudas et al., 1999; Schittkowski, 1987) and are often modified in order to fit the testing criteria of the algorithmic development. Specifically the number of test problems that we report in the table from online test libraries corresponds to problems which do not exceed a total of 300 in variables (continuous or discrete) or constraints, which is a reasonable bound for the current capabilities of state-of-the art methods in CDFO literature.

\section{Conclusions}

This review article presents and organizes contributions for MINLP and CDFO problems into their contribution domain. In addition, we show the interconnections between the contributions and their individual places within an algorithmic whole. It is clear that the theoretical advances in MINLP are significantly greater, due to the maturity of the literature, as well as the availability of explicit functions and derivatives of the mathematical models. On the other hand, global optimality for CDFO cannot be mathematically guaranteed within a finite number of steps due to the lack of analytical forms, derivatives and valid lower/upper bounds. However, the goal of CDFO algorithms is to locate improved local solutions without having exact knowledge of the form, convexity or smoothness of the underlying model. As a conclusion, if a problem can be entirely mathematically formulated in any of the forms which are provided in the MINLP definitions, the use of an appropriate MINLP solver is suggested. However, if any part of the model is unreliable, unknown or computationally expensive, then CDFO methods can be extremely effective. The significance of the areas that we address in this manuscript becomes evident through the large and diverse set of applications of both MINLP and CDFO found in the literature. This clearly demonstrates the importance of recent developments and their major impact on improving many real-world problems coming from industry and science. 


\section{Acknowledgements}

C. A. F and F. B. acknowledge financial support from the National Science Foundation (CBET-0827907, CBET-1263165). This work is also supported by a Royal Academy of Engineering Fellowship to R. M. Moreover, the authors would like to

thank the Reviewers for their suggestions which lead to significant improvements to the paper. 


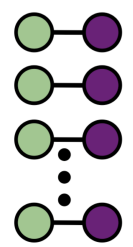

(a) Equation: Process Networks

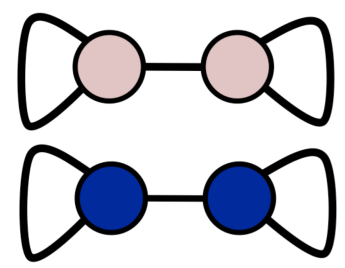

(c) Equation: Computational Geometry

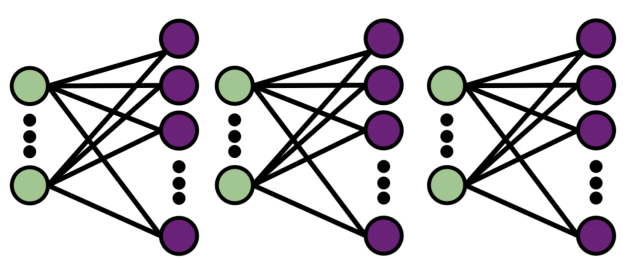

(b) Bilinear Terms: Process Networks

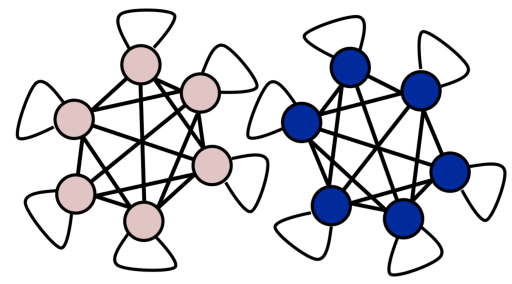

(d) Terms: Computational Geome-

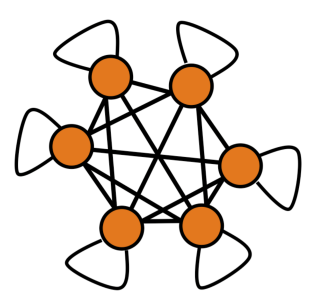

(e) Terms: MIQP

Figure 1: Undirected graph representations of quadratic nonlinearities in each equation and the collection of bilinear terms for several classes of MIQCQP (Misener et al., 2014b) 
Table 1: Common terms used as building blocks for solving MINLP

\begin{tabular}{|c|c|c|c|}
\hline Term Type & Prototype & Term Type & Prototype \\
\hline Constant & $c$ & Linear & $a \cdot x_{j}+b$ \\
\hline Bilinear / Quadratic & $x_{j 1} \cdot x_{j 2}$ & Absolute Value & $\left|a \cdot x_{j}+b\right|$ \\
\hline $\begin{array}{l}\text { Multivariate } \\
\text { Signomial }\end{array}$ & $x_{j 1}^{a_{1}} \cdot x_{j 2}^{a_{2}} \cdots x_{j n}^{a_{n}}$ & $\begin{array}{l}\text { Univariate } \\
\text { Signomial }\end{array}$ & $x_{j}^{a}$ \\
\hline Exponential & $e^{a \cdot x_{j}+b}$ & Logarithmic & $\log \left(a \cdot x_{j}+b\right)$ \\
\hline Trigonometric $^{\dagger}$ & $\sin \left(a \cdot x_{j}+b\right)$ & $\operatorname{Max} / \operatorname{Min}^{\dagger}$ & $\begin{array}{r}\max \left(a \cdot x_{j 1}+b\right. \\
\left.a \cdot x_{j 2}+b\right)\end{array}$ \\
\hline
\end{tabular}

Composite All solvers implicitly support compositions via auxiliary variables; some solvers also handle composite functions explicitly

\begin{tabular}{ll}
\hline Absolute Power & $\mathrm{lhs} \leq \operatorname{sign}(x+a)|x+a|^{n}+c z \leq \mathrm{rhs}$ \\
Exponent $^{\ddagger}$ & $\left(a_{1} \cdot x_{j}+b_{1}\right) \cdot e^{a_{2} \cdot x_{j}+b_{2}}$ \\
Fractional $^{\ddagger}$ & $\frac{a_{1} \cdot x_{j}+b_{1}}{a_{2} \cdot x_{j}+b_{2}}$ \\
Logarithm $^{\ddagger}$ &,$\left(a_{1} x_{j}+b_{1}\right) \log \left(a_{2} x_{j}+b_{2}\right)$ \\
General $\mathscr{C}^{2 \star}$ & $f_{i}(\boldsymbol{x})$
\end{tabular}

\footnotetext{
${ }^{\dagger}$ Only in: $\alpha \mathrm{BB}$ (Adjiman et al., 1998b); Couenne (Belotti et al., 2009); LINDO (Gau and Schrage, 2003)

- Only in: SCIP (Vigerske, 2012)

$¥$ Only in: ANTIGONE (Misener and Floudas, 2014a)

* Only in: $\alpha \mathrm{BB}$ (Adjiman et al., 1998b); $\alpha \mathrm{SGO} / \alpha \mathrm{R}$ (Lundell et al., 2013); these codes require that an underlaying nonlinear subsolver has the relevant functions
} 


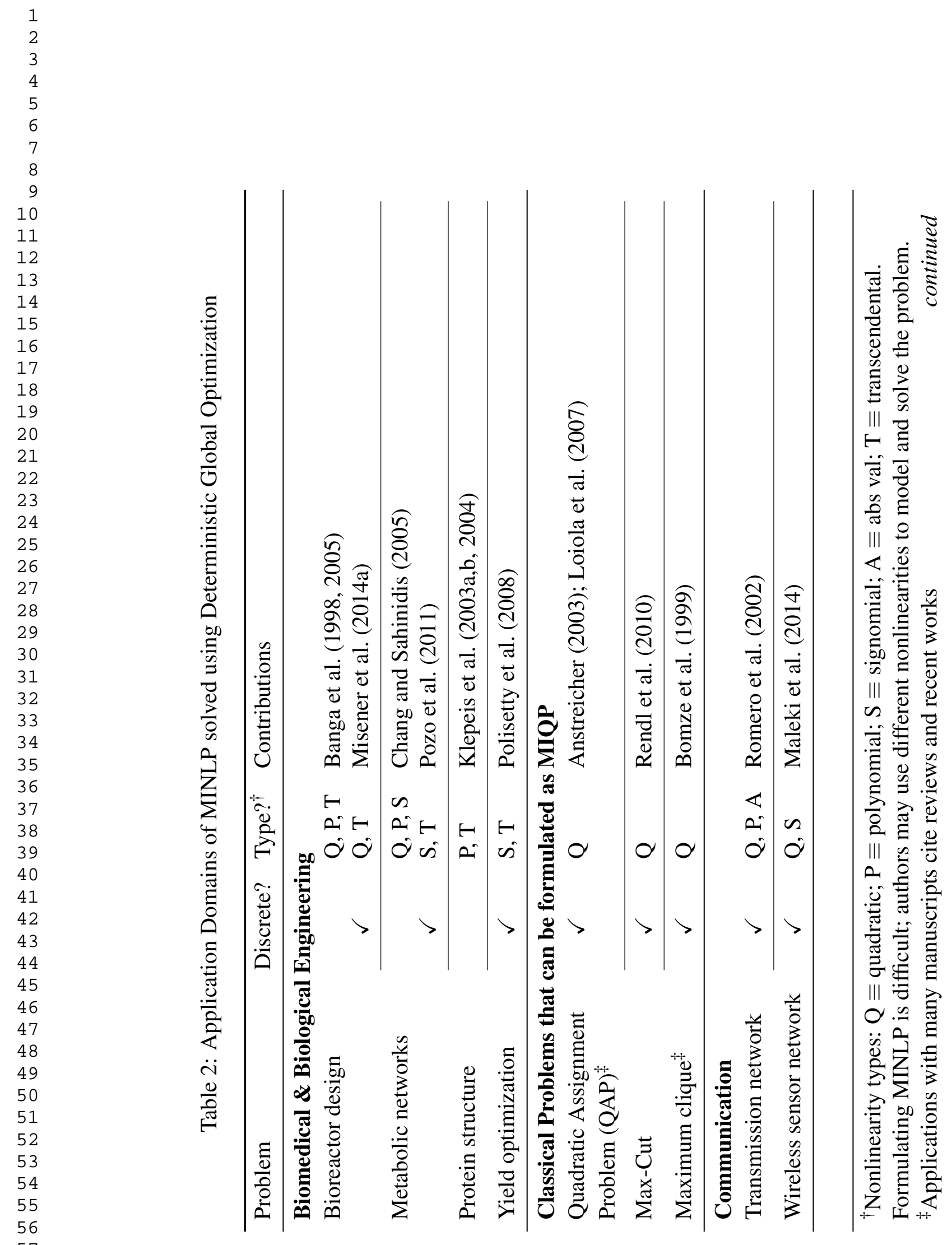




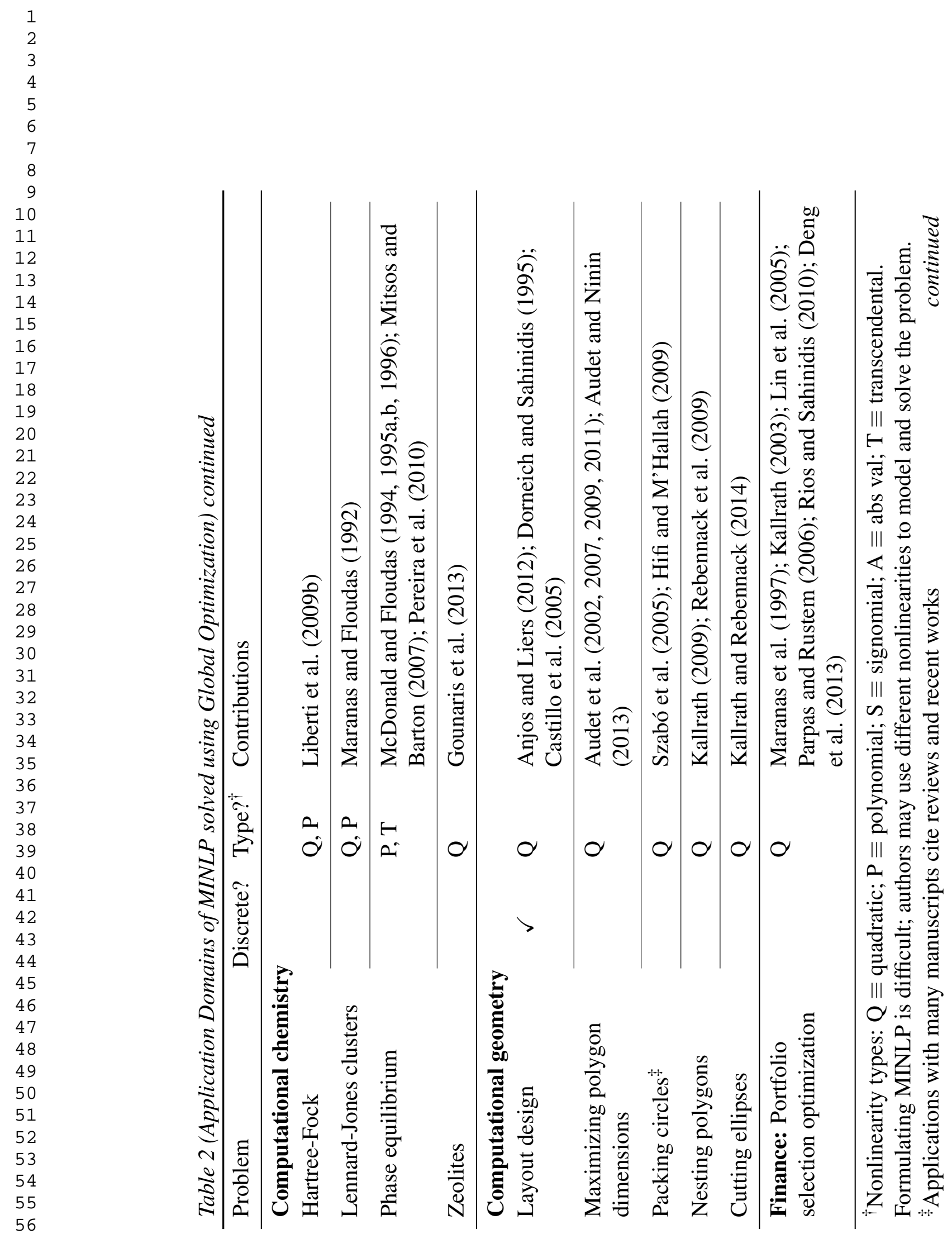




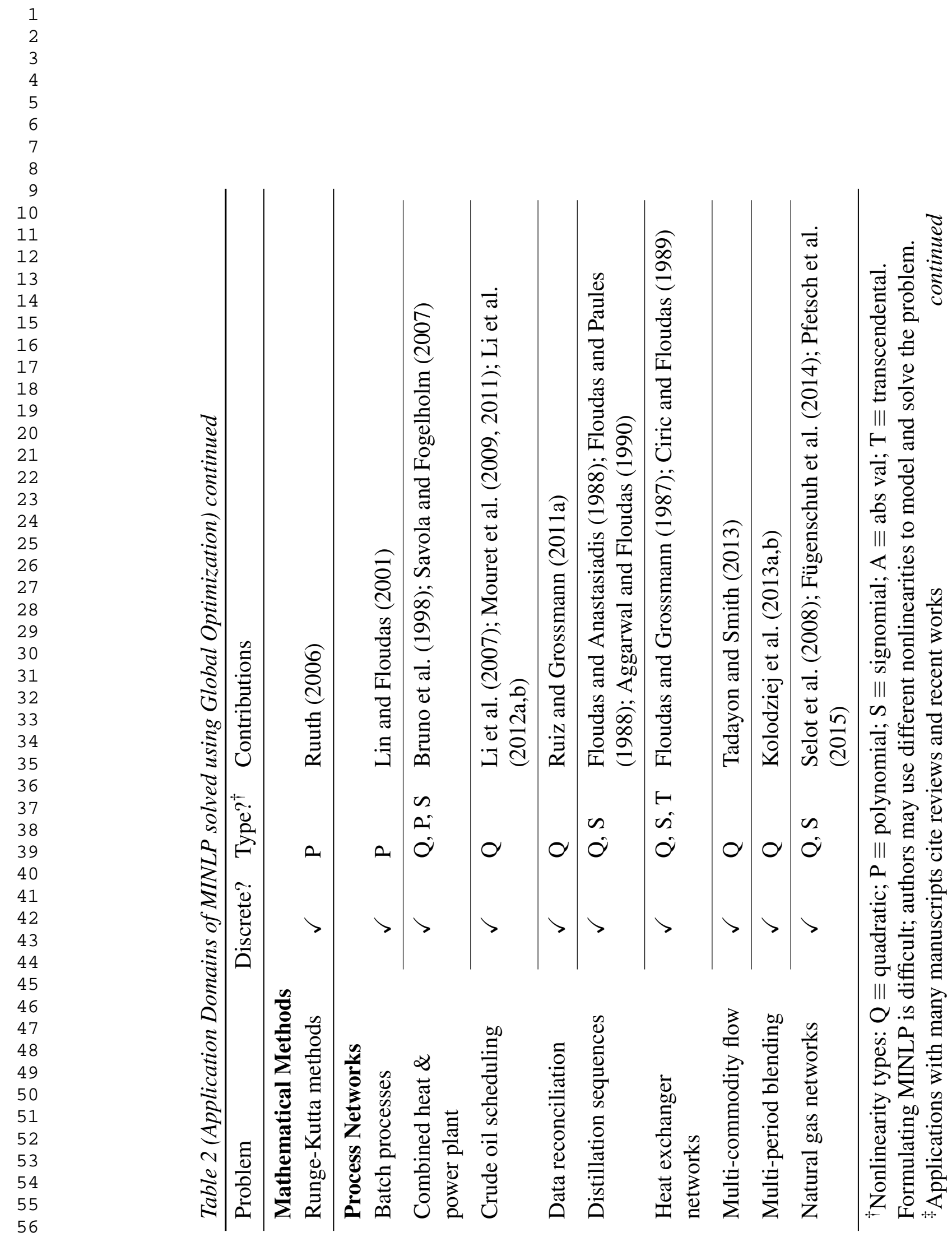




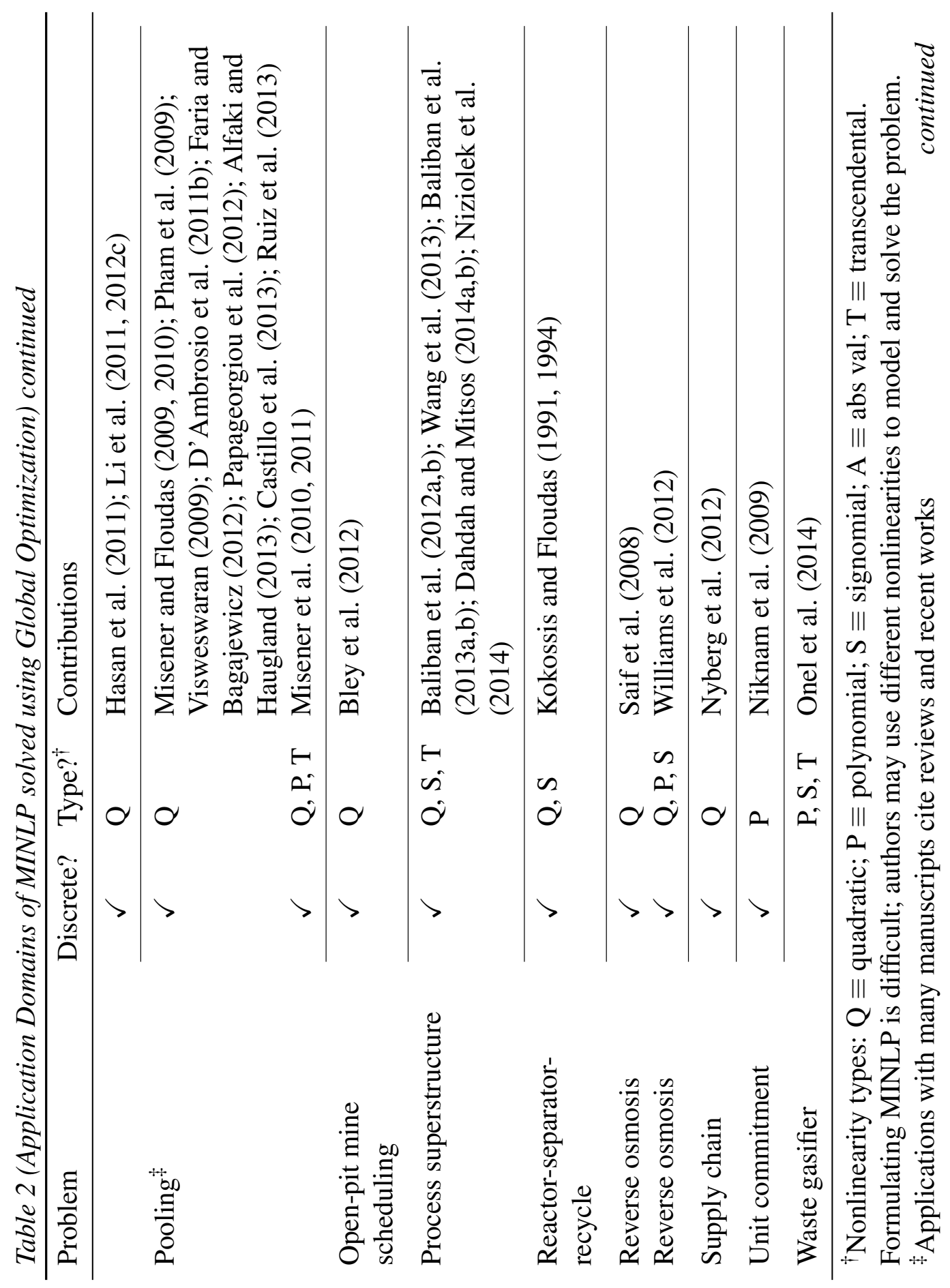




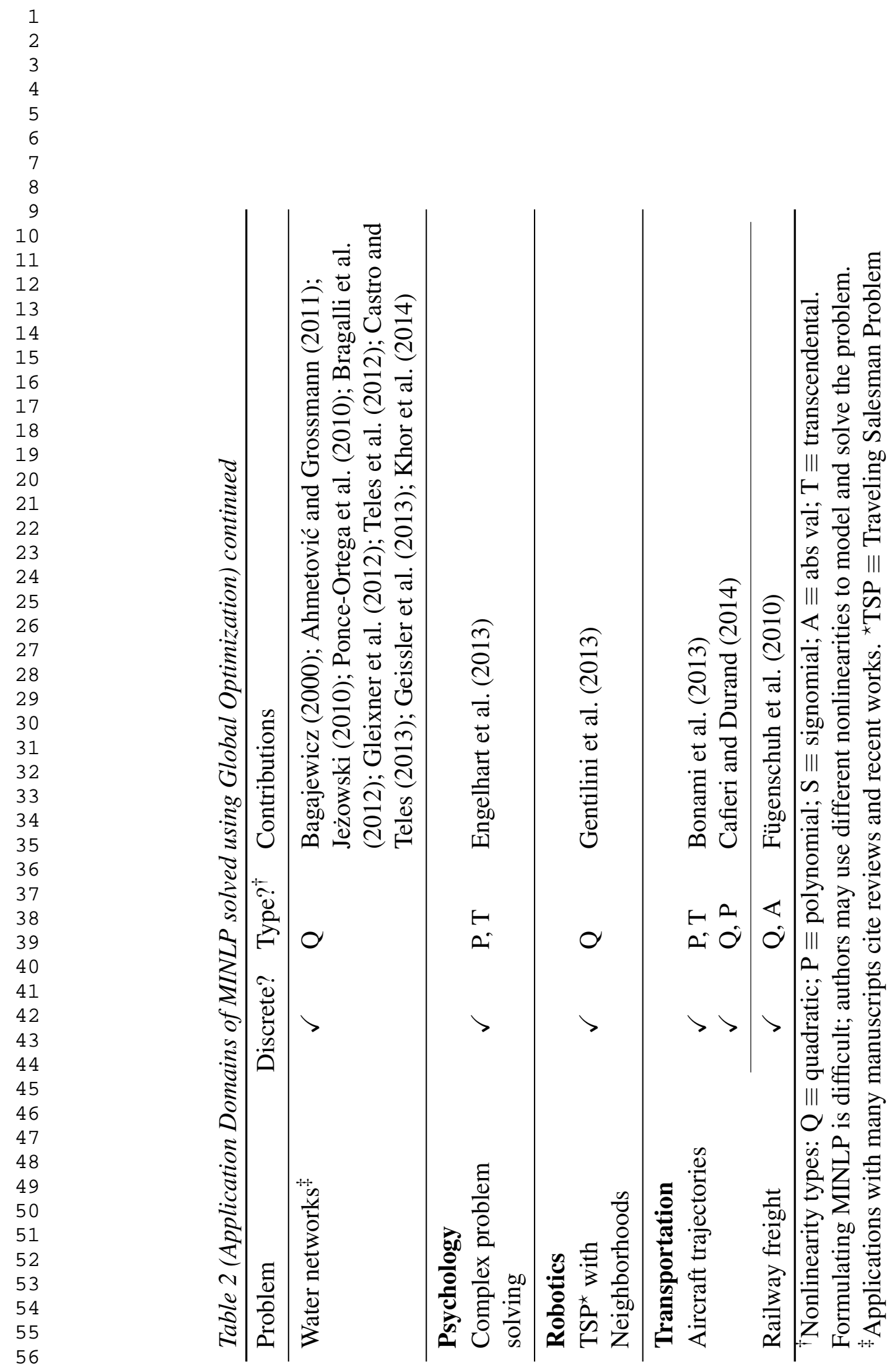




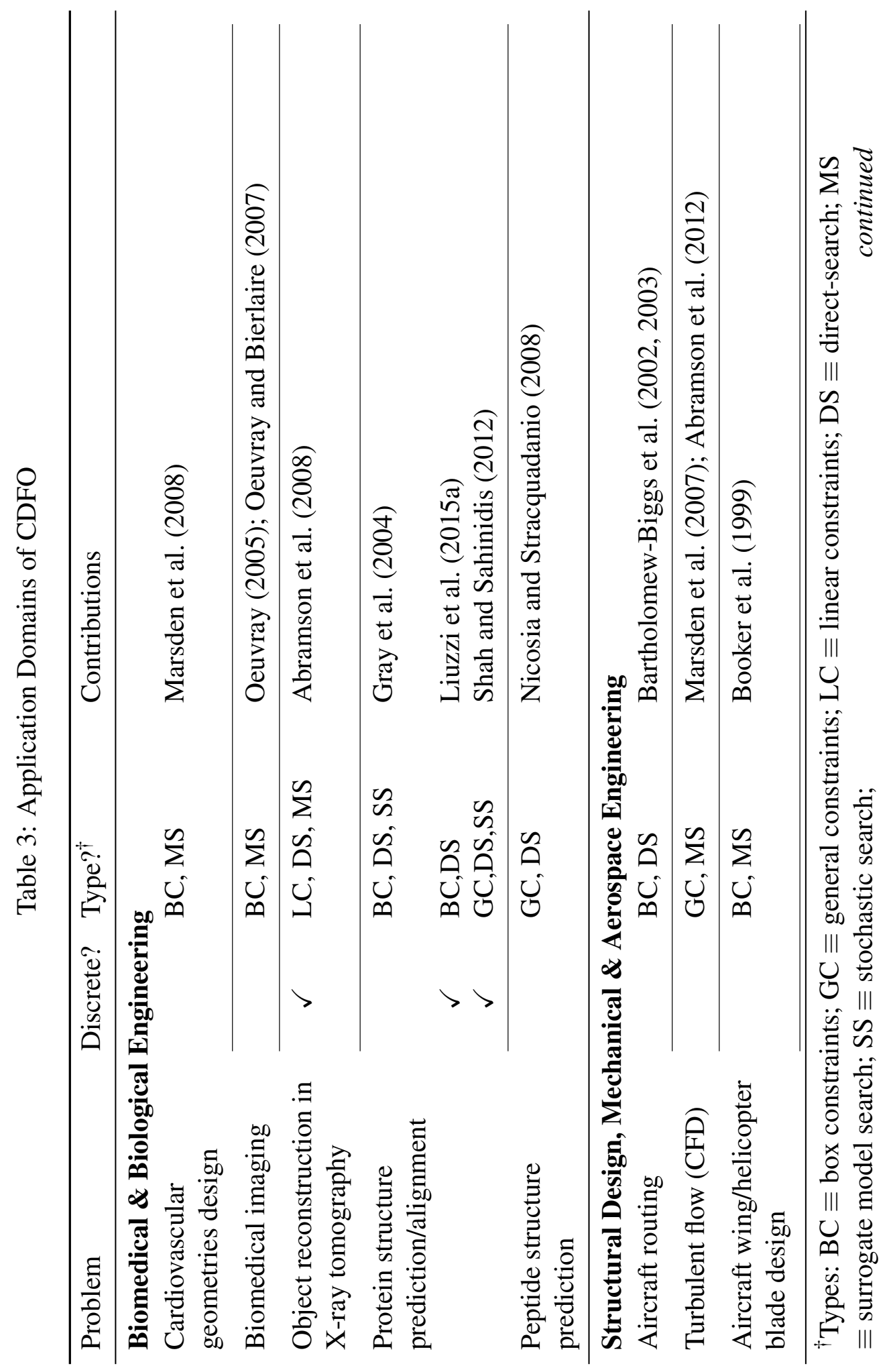


1

2
3

4

5

7

8
9

10

11

12

13

14

15

16

17
18

19

20

21

22

23

24
25

26

27

28

29
30

31

32

33

34

35

36

37

38

39

40

41

42

43

44

45

46

47

48

49
50

51

52

53

54

55

56

57

58

59

60

61

62

63

64

65

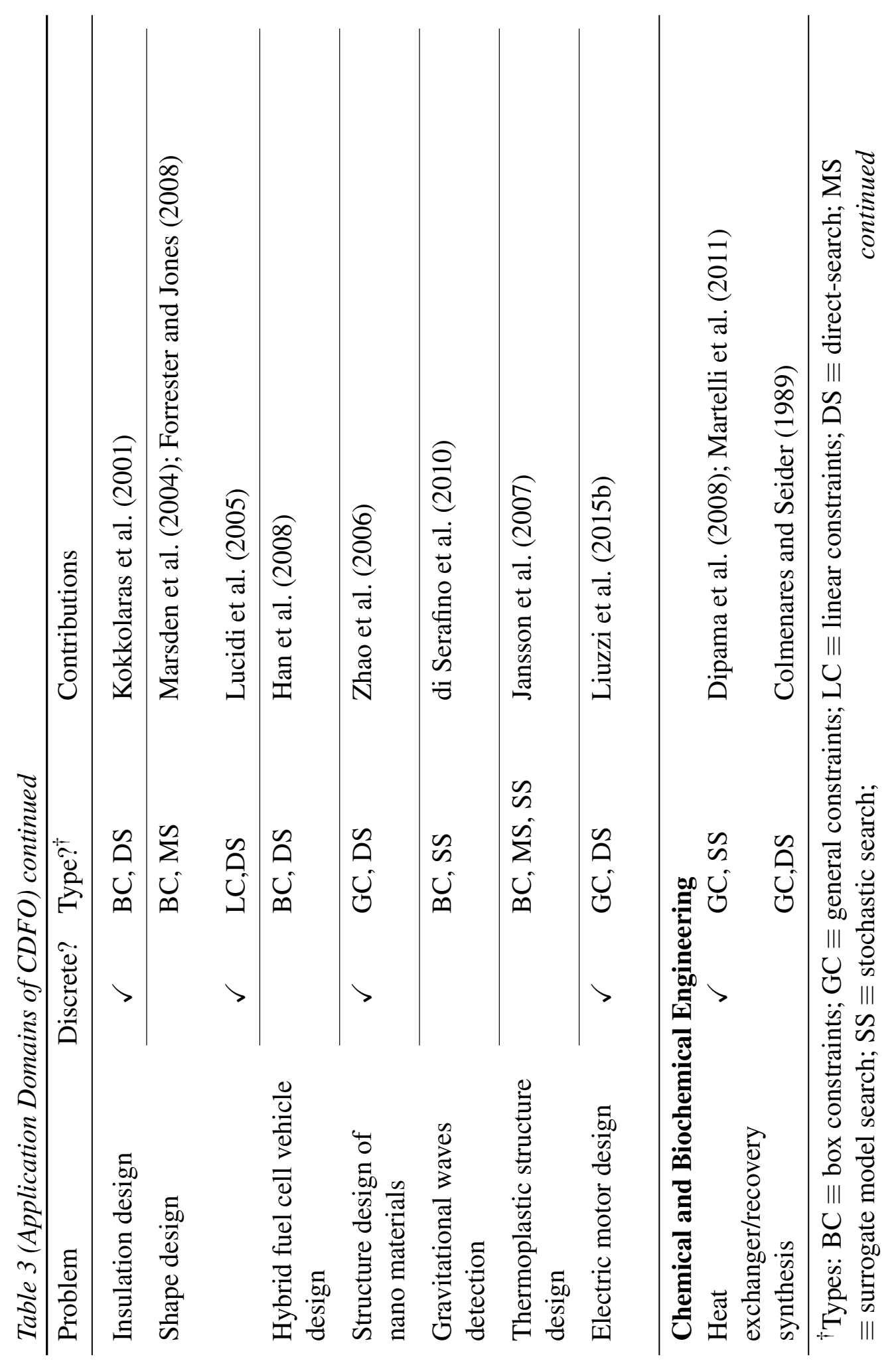




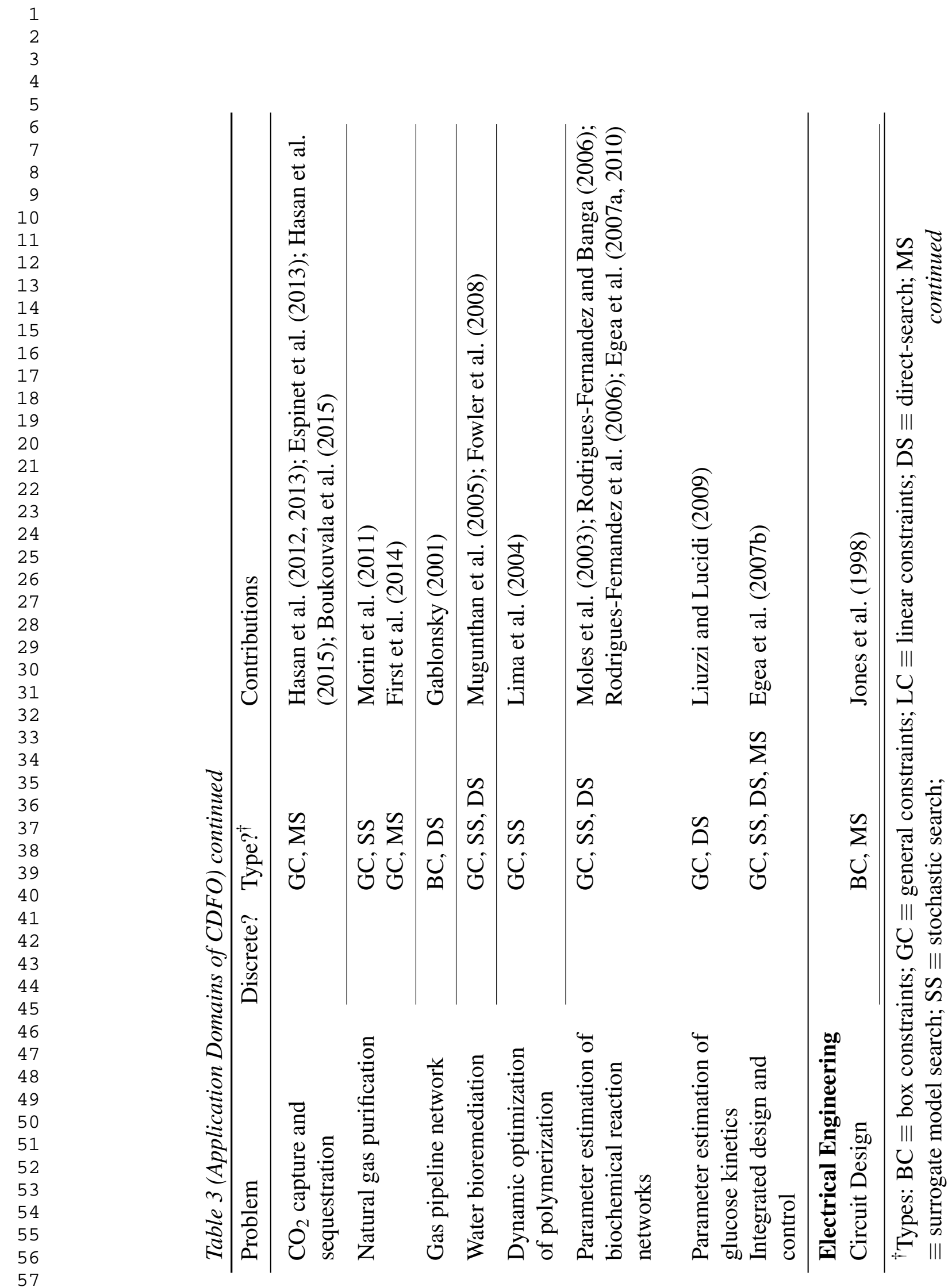




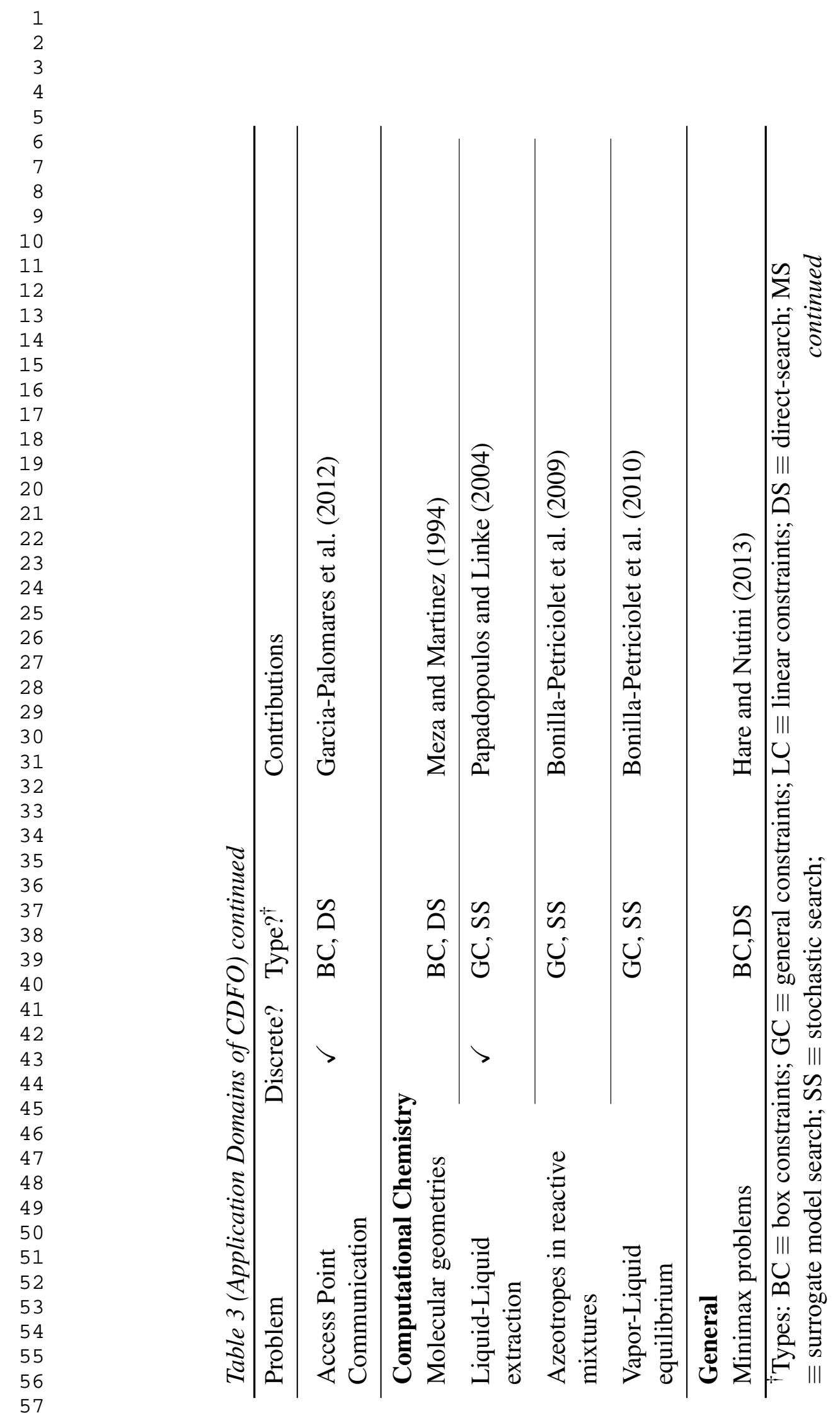

58

59

61

62

63

64 
Table 4: Overview of Major Reviews, Textbooks and Edited Books for MINLP

\begin{tabular}{|c|c|c|}
\hline Type & Reference & Contribution \\
\hline \multirow[t]{13}{*}{$\begin{array}{l}\text { Major } \\
\text { Reviews }\end{array}$} & $\begin{array}{l}\text { Bomze et al. } \\
(1999)\end{array}$ & $\begin{array}{l}\text { Maximum clique problem, can be formulated } \\
\text { as an MIQCQP }\end{array}$ \\
\hline & $\begin{array}{l}\text { Anstreicher } \\
(2003)\end{array}$ & $\begin{array}{l}\text { Quadratic assignment problem, an important } \\
\text { application of MIQCQP }\end{array}$ \\
\hline & $\begin{array}{l}\text { Biegler and } \\
\text { Grossmann } \\
(2004)\end{array}$ & Retrospective on optimization \\
\hline & $\begin{array}{l}\text { Grossmann and } \\
\text { Biegler (2004) }\end{array}$ & Future perspective on optimization \\
\hline & $\begin{array}{l}\text { Floudas et al. } \\
(2005)\end{array}$ & $\begin{array}{l}\text { Algorithms for deterministic global } \\
\text { optimization; includes MINLP }\end{array}$ \\
\hline & $\begin{array}{l}\text { Loiola et al. } \\
(2007)\end{array}$ & $\begin{array}{l}\text { Quadratic assignment problem, an important } \\
\text { application of MIQCQP }\end{array}$ \\
\hline & $\begin{array}{l}\text { Floudas and } \\
\text { Gounaris (2009) }\end{array}$ & $\begin{array}{l}\text { Deterministic global optimization for years } \\
1998-2008\end{array}$ \\
\hline & $\begin{array}{l}\text { Leyffer et al. } \\
(2009)\end{array}$ & Models; algorithms; software for MINLP \\
\hline & $\begin{array}{l}\text { Misener and } \\
\text { Floudas (2009) }\end{array}$ & $\begin{array}{l}\text { Models and algorithms for pooling problems, } \\
\text { an important application of MIQCQP }\end{array}$ \\
\hline & $\begin{array}{l}\text { Bussieck and } \\
\text { Vigerske (2010) }\end{array}$ & MINLP solver software \\
\hline & $\begin{array}{l}\text { Hemmecke et al. } \\
(2010)\end{array}$ & $\begin{array}{l}\text { Nonlinear integer programming; linear } \\
\text { constraints }\end{array}$ \\
\hline & $\begin{array}{l}\text { Grossmann and } \\
\text { Guillén-Gosálbez } \\
\text { (2010) }\end{array}$ & $\begin{array}{l}\text { Mathematical optimization for sustainability; } \\
\text { includes discussion of MINLP algorithms and } \\
\text { applications }\end{array}$ \\
\hline & Bomze (2012) & $\begin{array}{l}\text { Copositive optimization; has applications to } \\
\text { MINLP }\end{array}$ \\
\hline
\end{tabular}

continued on the next page 


\begin{tabular}{|c|c|c|}
\hline Type & Reference & Contribution \\
\hline & Burer and & Solving strategies (mostly deterministic) for \\
\hline & Letchford (2012) & MINLP; focus on quadratic nonlinearities \\
\hline & $\begin{array}{l}\text { Burer and Saxena } \\
(2012)\end{array}$ & Influence of MILP in solving MIQCQP \\
\hline & $\begin{array}{l}\text { Floudas et al. } \\
\text { (2012) }\end{array}$ & $\begin{array}{l}\text { Hybrid energy processes; includes many } \\
\text { MINLP applications }\end{array}$ \\
\hline & $\begin{array}{l}\text { Grossmann } \\
(2012)\end{array}$ & $\begin{array}{l}\text { Mathematical optimization for enterprise-wide } \\
\text { optimization; includes MINLP discussion }\end{array}$ \\
\hline & $\begin{array}{l}\text { Misener and } \\
\text { Floudas (2012a) }\end{array}$ & $\begin{array}{l}\text { Algorithms and implementations for global } \\
\text { optimization of MIQCQP and MINLP }\end{array}$ \\
\hline & $\begin{array}{l}\text { Yuan and Chen } \\
\text { (2012) }\end{array}$ & $\begin{array}{l}\text { Process synthesis; includes discussion of } \\
\text { MINLP }\end{array}$ \\
\hline & $\begin{array}{l}\text { Belotti et al. } \\
(2013 b)\end{array}$ & $\begin{array}{l}\text { Models, applications, and solving strategies for } \\
\text { MINLP; chapter on deterministic methods }\end{array}$ \\
\hline & $\begin{array}{l}\text { D'Ambrosio and } \\
\text { Lodi (2013) }\end{array}$ & $\begin{array}{l}\text { Algorithms and software for MINLP; includes } \\
\text { discussion of deterministic tools }\end{array}$ \\
\hline & $\begin{array}{l}\text { Harjunkoski et al. } \\
\text { (2014) }\end{array}$ & $\begin{array}{l}\text { Scheduling optimization methods; includes } \\
\text { discussion of MINLP and deterministic tools }\end{array}$ \\
\hline \multirow{6}{*}{$\begin{array}{l}\text { Texts / } \\
\text { Mono- } \\
\text { graphs }\end{array}$} & Floudas (1995) & Introduction to MINLP \\
\hline & $\begin{array}{l}\text { Horst and Tuy } \\
(1996)\end{array}$ & Global optimization \\
\hline & $\begin{array}{l}\text { Sherali and } \\
\text { Adams (1999) }\end{array}$ & Reformulation-linearization approach \\
\hline & Floudas (2000) & Deterministic global optimization \\
\hline & $\begin{array}{l}\text { Tawarmalani and } \\
\text { Sahinidis } \\
\text { (2002b) }\end{array}$ & $\begin{array}{l}\text { Deterministic global optimization of MINLP; } \\
\text { algorithmic framework }\end{array}$ \\
\hline & Nowak (2005) & Global optimization of MINLP; LaGO \\
\hline
\end{tabular}


Table 4 (Major Reviews; Textbooks; Edited Books) continued

\begin{tabular}{|c|c|c|}
\hline Type & Reference & Contribution \\
\hline & $\begin{array}{l}\text { Locatelli and } \\
\text { Schoen (2013) }\end{array}$ & Global optimization \\
\hline \multirow[t]{7}{*}{$\begin{array}{l}\text { Edited } \\
\text { Books }\end{array}$} & $\begin{array}{l}\text { Floudas and } \\
\text { Pardalos (1992) }\end{array}$ & Recent Advances In Global Optimization \\
\hline & $\begin{array}{l}\text { Floudas and } \\
\text { Pardalos (1995) }\end{array}$ & $\begin{array}{l}\text { State-of-the-art in global } \\
\text { optimization-computational methods and } \\
\text { applications - preface }\end{array}$ \\
\hline & $\begin{array}{l}\text { Floudas and } \\
\text { Pardalos (1996) }\end{array}$ & $\begin{array}{l}\text { State of the Art In Global Optimization: } \\
\text { Computational Methods and Applications }\end{array}$ \\
\hline & $\begin{array}{l}\text { Floudas and } \\
\text { Pardalos (2000) }\end{array}$ & $\begin{array}{l}\text { Optimization in Computational Chemistry and } \\
\text { Molecular Biology: Local and Global } \\
\text { Approaches }\end{array}$ \\
\hline & $\begin{array}{l}\text { Floudas and } \\
\text { Pardalos (2004) }\end{array}$ & Frontiers in Global Optimization \\
\hline & $\begin{array}{l}\text { Liberti and } \\
\text { Maculan (2006) }\end{array}$ & Deterministic global optimization \\
\hline & $\begin{array}{l}\text { Lee and Leyffer } \\
\text { (2012) }\end{array}$ & MINLP \\
\hline
\end{tabular}


Table 5: Overview of Major Reviews, Textbooks and Edited Books for CDFO

\begin{tabular}{|c|c|c|}
\hline Type & Reference & Contribution \\
\hline \multirow[t]{4}{*}{$\begin{array}{l}\text { Major } \\
\text { Reviews }\end{array}$} & $\begin{array}{l}\text { Kolda et al. } \\
\text { (2003) }\end{array}$ & $\begin{array}{l}\text { Extensive review of direct-search methods for } \\
\text { BCDFO and CDFO }\end{array}$ \\
\hline & $\begin{array}{l}\text { Das and } \\
\text { Suganthan (2011) }\end{array}$ & $\begin{array}{l}\text { Review of Differential Evolution/ Stochastic } \\
\text { CDFO methods }\end{array}$ \\
\hline & $\begin{array}{l}\text { Rios and } \\
\text { Sahinidis (2013) }\end{array}$ & $\begin{array}{l}\text { Review of BCDFO and extensive comparison } \\
\text { of algorithmic performance }\end{array}$ \\
\hline & $\begin{array}{l}\text { Martelli and } \\
\text { Amaldi (2014) }\end{array}$ & $\begin{array}{l}\text { Brief review of CDFO methods;coupling with } \\
\text { stochastic methods }\end{array}$ \\
\hline \multirow{2}{*}{$\begin{array}{l}\text { Texts/ } \\
\text { Mono- } \\
\text { graphs }\end{array}$} & $\begin{array}{l}\text { Forrester et al. } \\
\text { (2008) }\end{array}$ & Model-based box-constrained CDFO problems \\
\hline & $\begin{array}{l}\text { Conn et al. } \\
(2009 b)\end{array}$ & $\begin{array}{l}\text { Introduction to derivative-free optimization } \\
\text { theory, focus on local-search methods }\end{array}$ \\
\hline \multirow[t]{2}{*}{$\begin{array}{l}\text { Edited } \\
\text { Books }\end{array}$} & Zabinsky (2003) & Stochastic CDFO theory and algorithms \\
\hline & $\begin{array}{l}\text { Koziel and Yang } \\
\text { (2011) }\end{array}$ & $\begin{array}{l}\text { Topics on direct-search, surrogate-based and } \\
\text { stochastic CDFO optimization }\end{array}$ \\
\hline
\end{tabular}


1 3 4 5 7 8
9 10 11 12 13

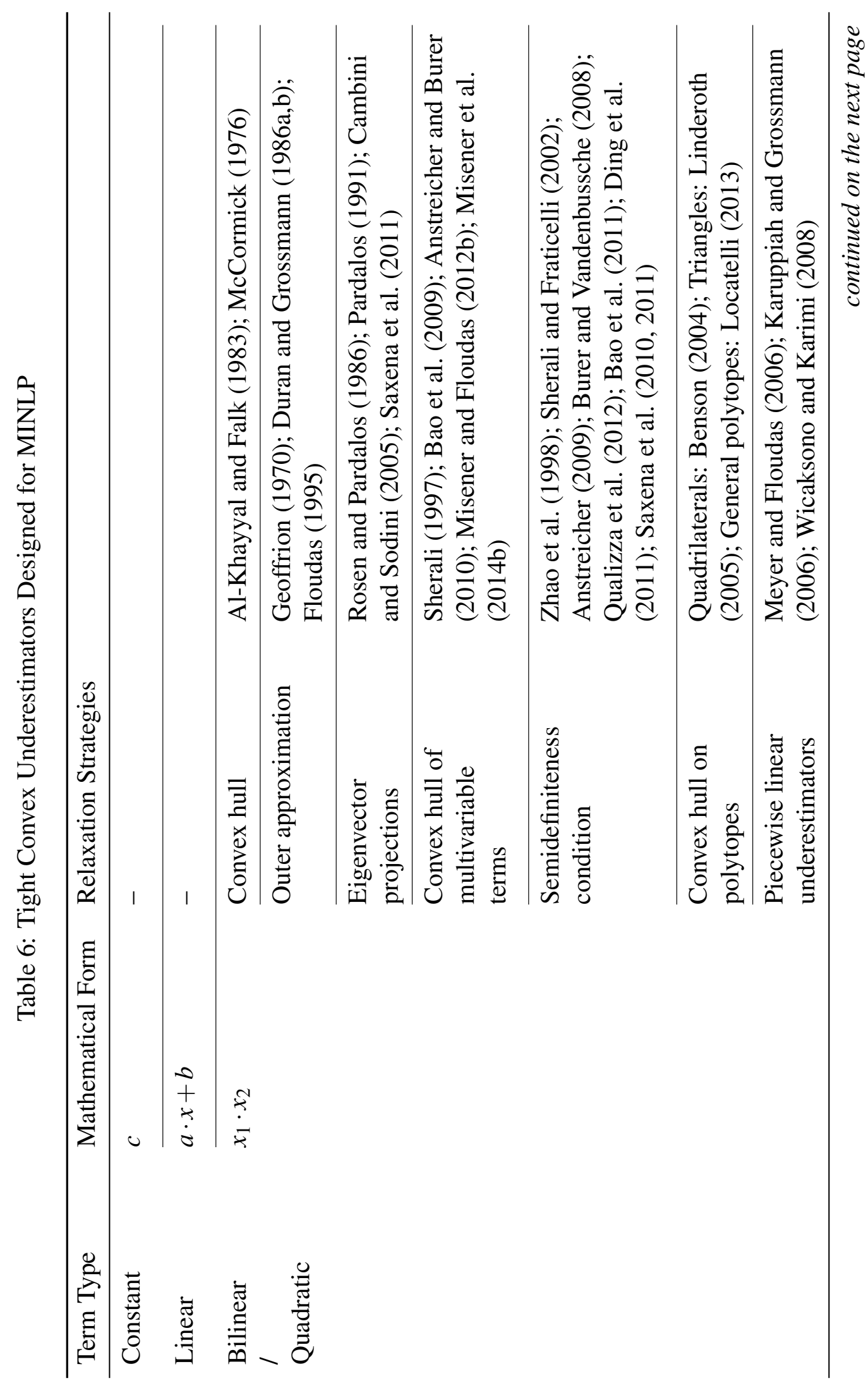




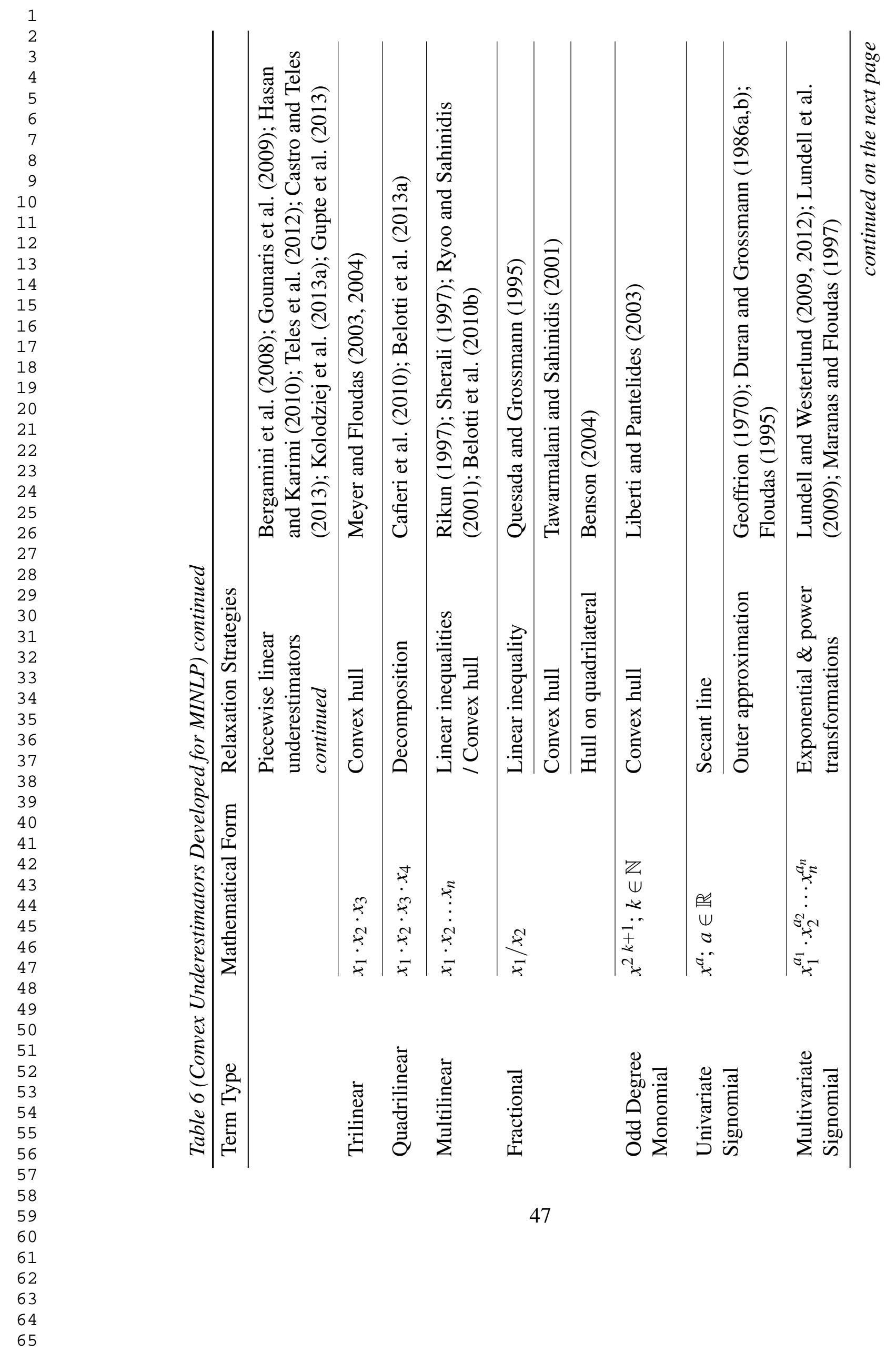




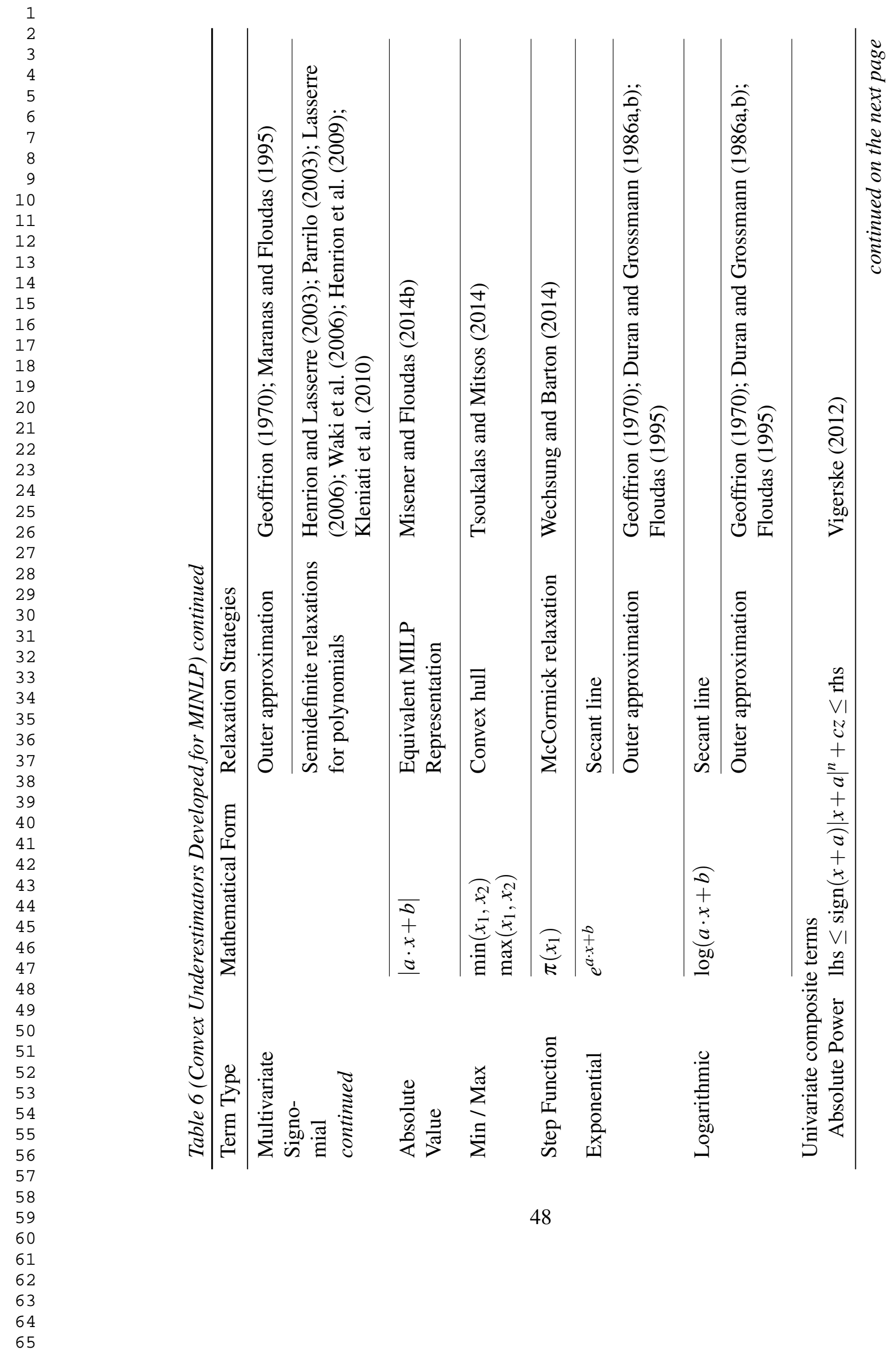




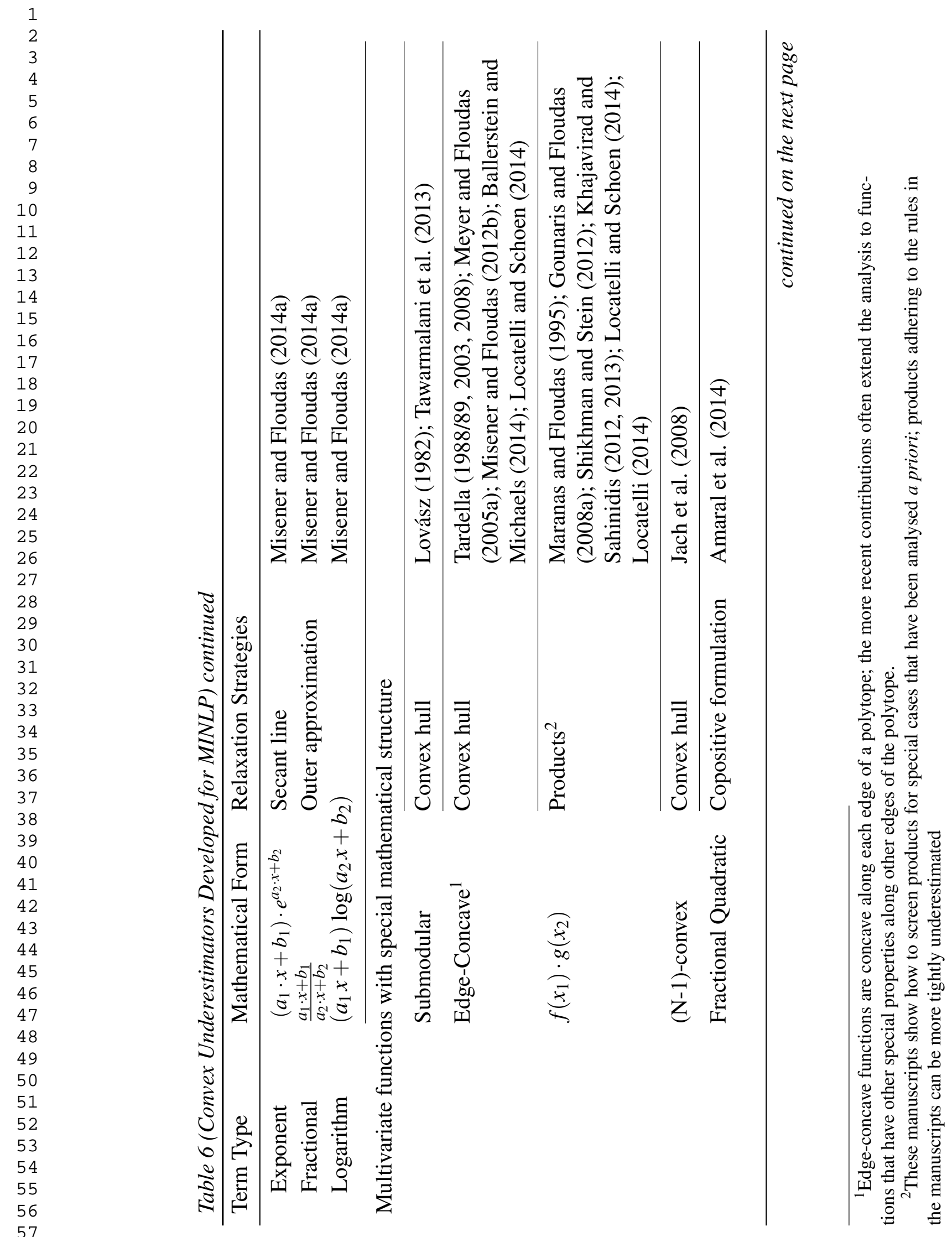


1 3 4 5 6 8 9
10 11 12 13 14 15 16 17 18 19 20

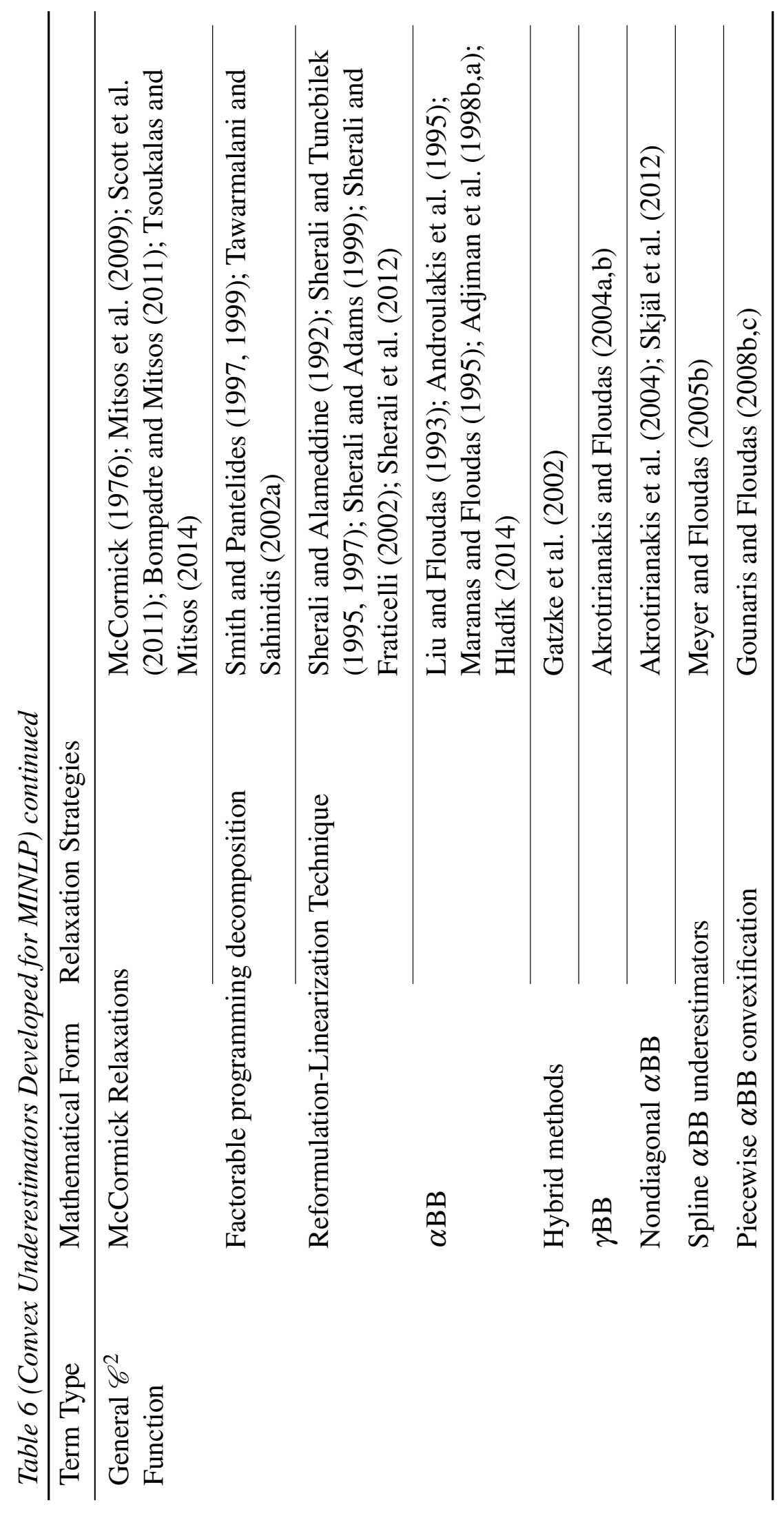


Table 7: Branching Algorithms Developed for MINLP

\begin{tabular}{ll}
\hline Reference & Contribution \\
\hline $\begin{array}{l}\text { Branch on variable with } \\
\text { the greatest error }\end{array}$ & Adjiman et al. (1998b,a); Audet et al. (2000) \\
\hline Ellipsoidal branching & Hoai An (2000); Hager and Phan (2009) \\
\hline Violation transfer & Tawarmalani and Sahinidis (2002b, 2004) \\
\hline Branching on triangles & Linderoth (2005); Locatelli (2015) \\
\hline Reliability branching & Achterberg et al. (2005); Belotti et al. (2009) \\
\hline $\begin{array}{l}\text { Uncover hidden } \\
\text { convexity in } \\
\text { subproblems }\end{array}$ & Gentilini et al. (2013) \\
\hline Undercover branching & Berthold and Gleixner (2013b) \\
\hline
\end{tabular}


Table 8: Bounding Algorithms Developed for MINLP

\begin{tabular}{|c|c|}
\hline Algorithm type & Reference \\
\hline $\begin{array}{l}\text { RLT-based bounds } \\
\text { tightening }\end{array}$ & $\begin{array}{l}\text { Androulakis et al. (1995); Audet et al. (2000); Sherali } \\
\text { and Tuncbilek (1995) }\end{array}$ \\
\hline $\begin{array}{l}\text { Reduced-cost bounds } \\
\text { tightening }\end{array}$ & Ryoo and Sahinidis $(1995,1996)$ \\
\hline $\begin{array}{l}\text { Quadratic equation } \\
\text { constraint satisfaction }\end{array}$ & $\begin{array}{l}\text { Domes and Neumaier (2010, 2011); Granvilliers and } \\
\text { Benhamou (2006); Lebbah et al. (2005); Vigerske (2012) }\end{array}$ \\
\hline $\begin{array}{l}\text { Aggressive bounds } \\
\text { tightening }\end{array}$ & Belotti et al. (2009) \\
\hline $\begin{array}{l}\text { Tightening based on } \\
\text { equation pairs }\end{array}$ & Belotti (2013) \\
\hline Convergence properties & Belotti et al. (2013b) \\
\hline $\begin{array}{l}\text { Low-dimensional } \\
\text { edge-concave } \\
\text { aggregations }\end{array}$ & Misener and Floudas (2012b) \\
\hline Nonlinearities removal & Caprara and Locatelli (2010) \\
\hline $\begin{array}{l}\text { Propagating Lagrangian } \\
\text { bounds }\end{array}$ & Gleixner and Weltge (2013) \\
\hline
\end{tabular}




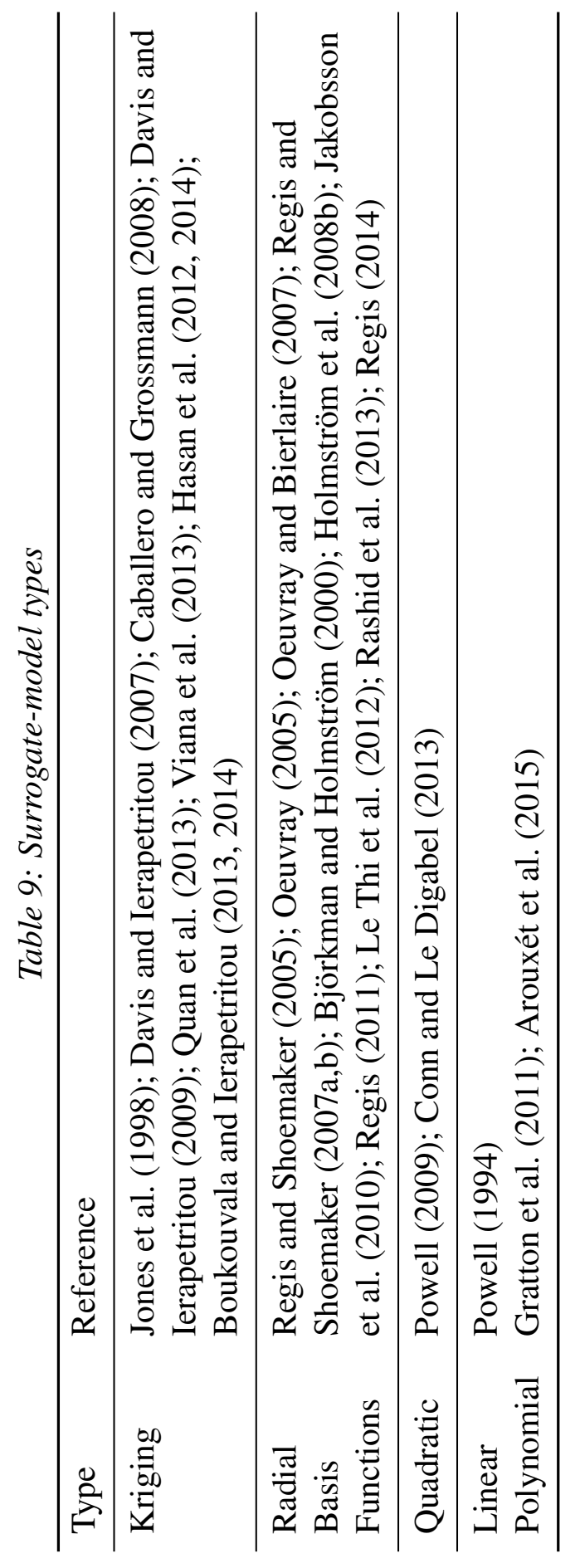


Table 10: Deterministic Global Optimization MINLP Solver Software

\begin{tabular}{ll}
\hline Solver & Web Link \\
\hline$\alpha \mathrm{BB}$ & titan.princeton.edu \\
$\alpha \mathrm{SGO} / \alpha \mathrm{R}$ & blogs.abo.fi/ose/ \\
ANTIGONE & helios.princeton.edu/ANTIGONE \\
BARON & archimedes.cheme.cmu.edu/?q=baron \\
Couenne & projects.coin-or.org/Couenne \\
GloMIQO & helios.princeton.edu/GloMIQO \\
LINDO & www.lindo.com \\
SCIP & scip.zib.de
\end{tabular}

See Table 11 for research advances related to developing this software

Table 11: Theoretical and Algorithmic Advances for Deterministic Global Optimization MINLP Solver Software

\begin{tabular}{|c|c|c|c|}
\hline Solver & Availability & References & Solver Feature \\
\hline \multirow[t]{7}{*}{$\alpha \mathrm{BB}$} & $\begin{array}{l}\text { Princeton } \\
\text { University }\end{array}$ & $\begin{array}{l}\text { Androulakis et al. (1995); } \\
\text { Maranas and Floudas (1995) }\end{array}$ & $\begin{array}{l}\text { Underestimators for general } \\
\mathrm{C}^{2} \text { functions }\end{array}$ \\
\hline & & Adjiman et al. (1998b,a) & Global NLP framework \\
\hline & & Adjiman et al. (2000) & Global MINLP framework \\
\hline & & $\begin{array}{l}\text { Akrotirianakis and Floudas } \\
(2004 a, b)\end{array}$ & Exponential underestimators \\
\hline & & $\begin{array}{l}\text { Akrotirianakis et al. (2004); } \\
\text { Skjäl et al. (2012) }\end{array}$ & $\begin{array}{l}\text { Nondiagonal Hessian } \\
\text { perturbation }\end{array}$ \\
\hline & & Meyer and Floudas (2005b) & Spline underestimators \\
\hline & & $\begin{array}{l}\text { Gounaris and Floudas } \\
(2008 b, c)\end{array}$ & Piecewise convexification \\
\hline$\alpha \mathrm{R}$ & $\begin{array}{l}\text { Abo } \\
\text { Akademi }\end{array}$ & $\begin{array}{l}\text { Lundell and Westerlund } \\
\text { (2012); Lundell et al. (2013) }\end{array}$ & Global MINLP framework \\
\hline
\end{tabular}


Table 11 (MINLP Solver Software) continued

\begin{tabular}{|c|c|c|c|}
\hline Solver & Availability & References & Solver Feature \\
\hline$\alpha \mathrm{SGO}$ & $\begin{array}{l}\text { Abo } \\
\text { Akademi }\end{array}$ & $\begin{array}{l}\text { Lundell and Westerlund } \\
\text { (2009); Lundell et al. (2009) }\end{array}$ & $\begin{array}{l}\text { Relaxations for signomial } \\
\text { terms }\end{array}$ \\
\hline \multirow[t]{4}{*}{$\begin{array}{l}\text { ANTI- } \\
\text { GONE }\end{array}$} & \multirow[t]{4}{*}{ GAMS } & $\begin{array}{l}\text { Misener and Floudas (2012b, } \\
\text { 2013) }\end{array}$ & Global MIQCQP framework \\
\hline & & Misener et al. (2014b) & Bilinear cutting planes \\
\hline & & Misener and Floudas (2014b) & Global MISO framework \\
\hline & & Misener and Floudas (2014a) & Global MINLP framework \\
\hline \multirow[t]{6}{*}{ BARON } & \multirow{6}{*}{$\begin{array}{l}\text { AIMMS; } \\
\text { GAMS; } \\
\text { MAT- } \\
\text { LAB; } \\
\text { YALMIP }\end{array}$} & $\begin{array}{l}\text { Sahinidis (1996); } \\
\text { Tawarmalani and Sahinidis } \\
(2002 a, b, 2004)\end{array}$ & Global MINLP framework \\
\hline & & $\begin{array}{l}\text { Ryoo and Sahinidis (1995, } \\
1996)\end{array}$ & $\begin{array}{l}\text { Range reduction } \\
\text { (Duality-based) }\end{array}$ \\
\hline & & $\begin{array}{l}\text { Ryoo and Sahinidis (2001) } \\
\text { Tawarmalani and Sahinidis } \\
(2001)\end{array}$ & $\begin{array}{l}\text { Range reduction (FBBT) } \\
\text { Relaxations for fractional } \\
\text { terms }\end{array}$ \\
\hline & & $\begin{array}{l}\text { Tawarmalani and Sahinidis } \\
(2005)\end{array}$ & Polyhedral Branch-\&-Cut \\
\hline & & $\begin{array}{l}\text { Bao et al. (2009); Zorn and } \\
\text { Sahinidis }(2013,2014 a)\end{array}$ & $\begin{array}{l}\text { Bilinear cutting planes } \\
\text { (including RLT) }\end{array}$ \\
\hline & & Zorn and Sahinidis (2014b) & Polynomial cutting planes \\
\hline \multirow[t]{3}{*}{ Couenne } & \multirow{3}{*}{$\begin{array}{l}\text { AMPL; } \\
\text { Coin-OR; } \\
\text { GAMS }\end{array}$} & Belotti et al. (2009) & MINLP \\
\hline & & $\begin{array}{l}\text { Belotti et al. (2010a, 2012); } \\
\text { Belotti (2013) }\end{array}$ & Range reduction (FBBT) \\
\hline & & Belotti et al. (2013a) & Quadrilinear terms \\
\hline $\begin{array}{l}\text { Glo- }^{3} \\
\text { MIQO }\end{array}$ & GAMS & $\begin{array}{l}\text { Misener and Floudas (2012b, } \\
\text { 2013); Misener et al. (2014b) }\end{array}$ & Global MIQCQP framework \\
\hline
\end{tabular}

continued on the next page

\footnotetext{
${ }^{3}$ ANTIGONE performs equivalently to GloMIQO when all nonlinearities are quadratic
} 
Table 11 (MINLP Solver Software) continued

\begin{tabular}{llll}
\hline Solver & Availability & References & Solver Feature \\
\cline { 2 - 4 } LINDO & $\begin{array}{l}\text { GAMS; } \\
\text { What'sBest! }\end{array}$ & $\begin{array}{l}\text { Gau and Schrage (2003); Lin } \\
\text { and Schrage (2009) }\end{array}$ & MINLP \\
\cline { 2 - 4 } SCIP & $\begin{array}{l}\text { GAMS; } \\
\text { NEOS; }\end{array}$ & $\begin{array}{l}\text { Achterberg (2009); } \\
\text { Achterberg et al. (2008) }\end{array}$ & MILP \\
\cline { 3 - 4 } & ZIMPL & Berthold et al. (2012b,a) & Global MIQCQP framework \\
\cline { 3 - 4 } & & Vigerske (2012) & Global MINLP framework \\
\cline { 3 - 4 } & & $\begin{array}{l}\text { Berthold and Gleixner } \\
(2013 b)\end{array}$ & Branching Variable Choice \\
\cline { 3 - 4 } & & Gleixner and Weltge (2013) & Range reduction (OBBT) \\
\cline { 3 - 4 } & & $\begin{array}{l}\text { Berthold and Gleixner } \\
(2013 a) ; \text { Berthold (2013) }\end{array}$ & Primal heuristics \\
\hline & &
\end{tabular}




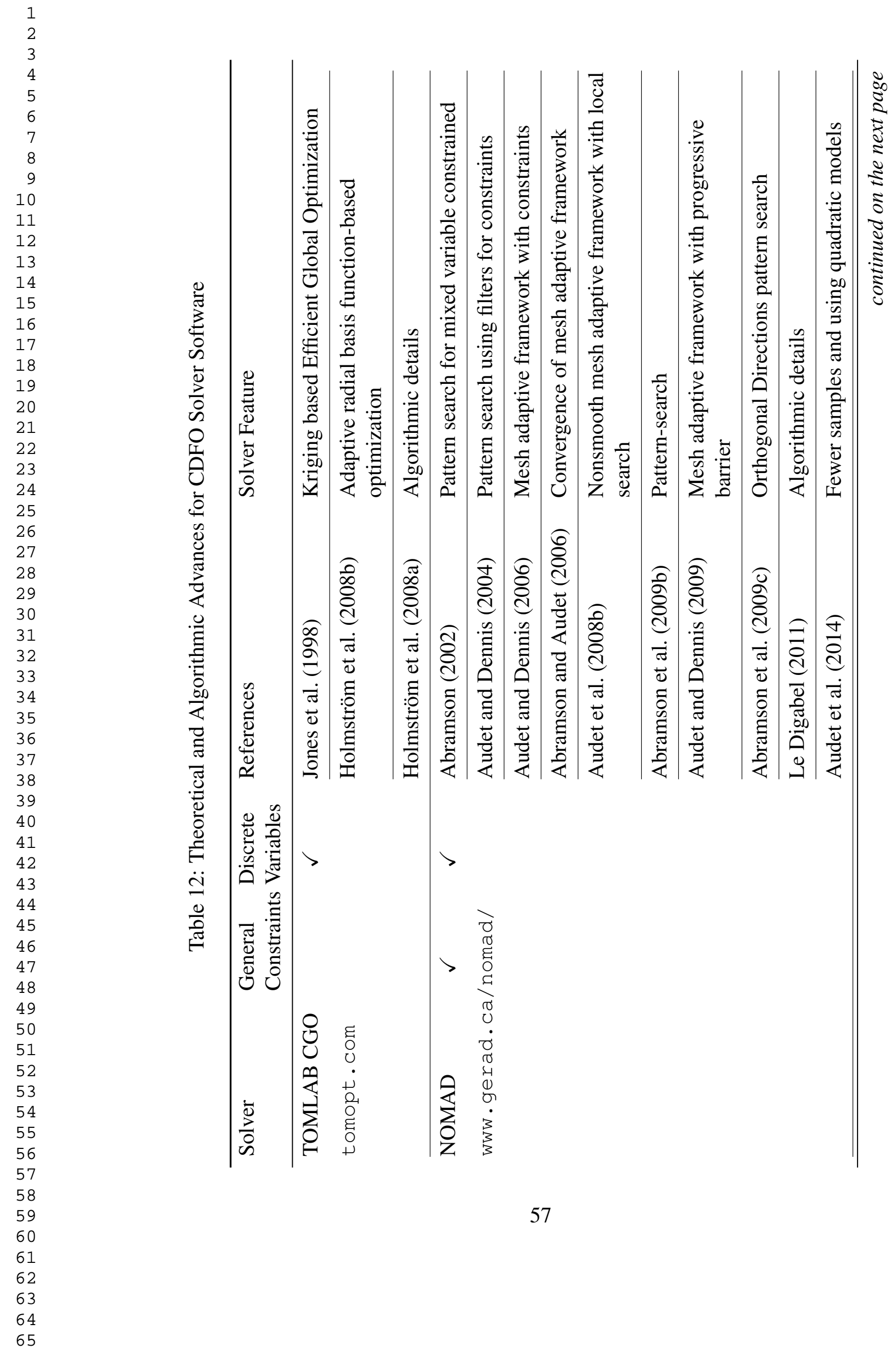




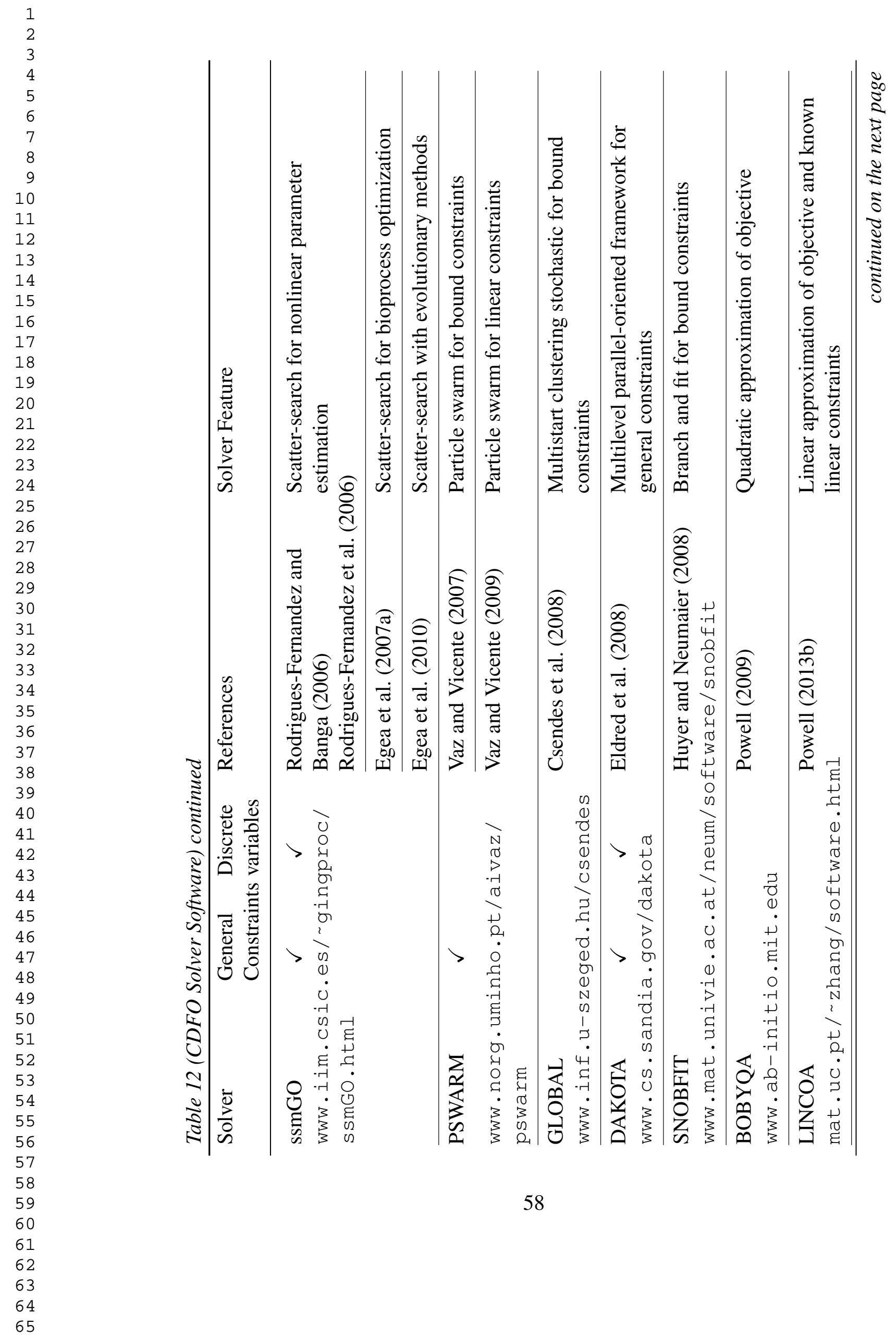




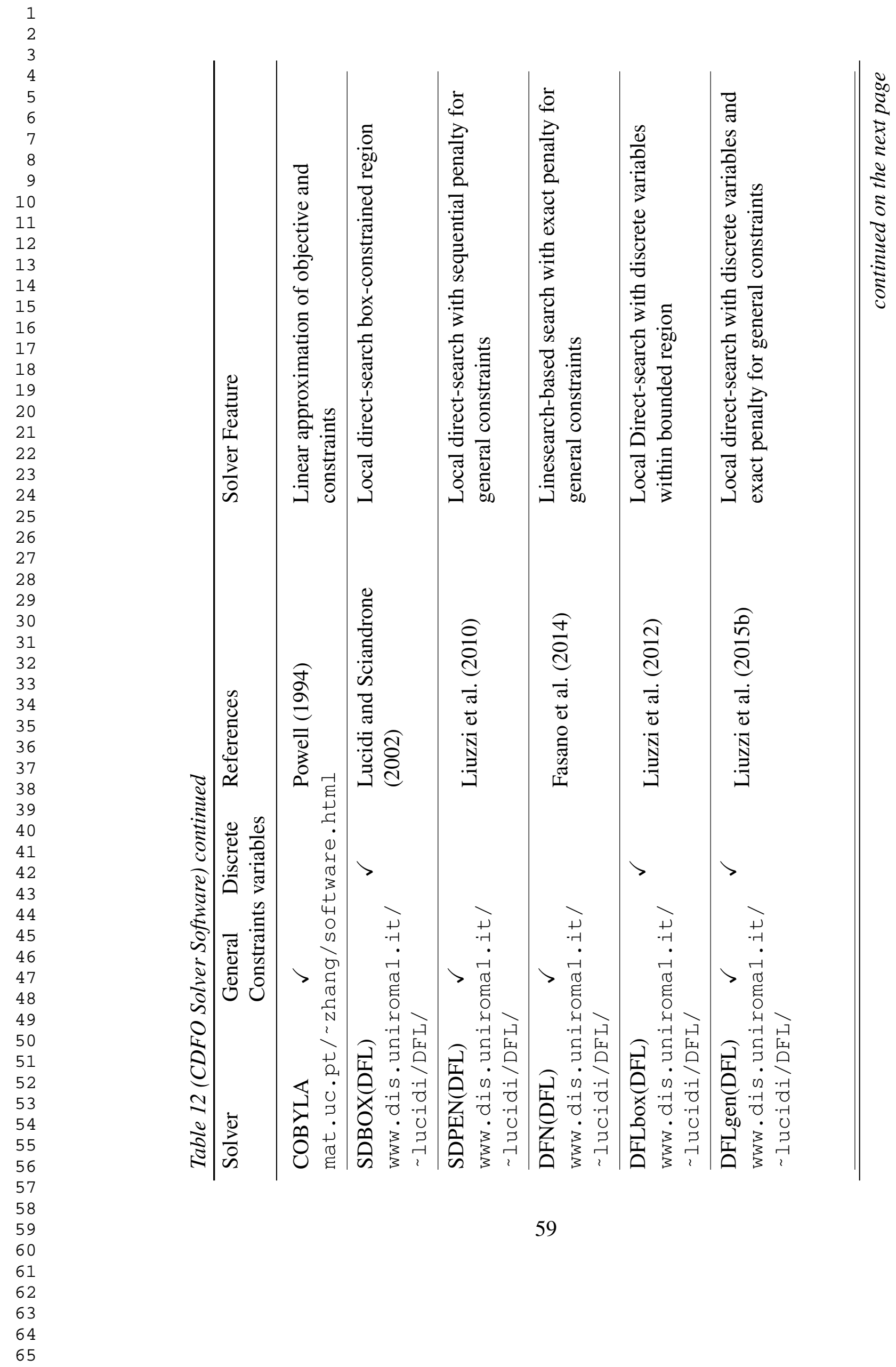




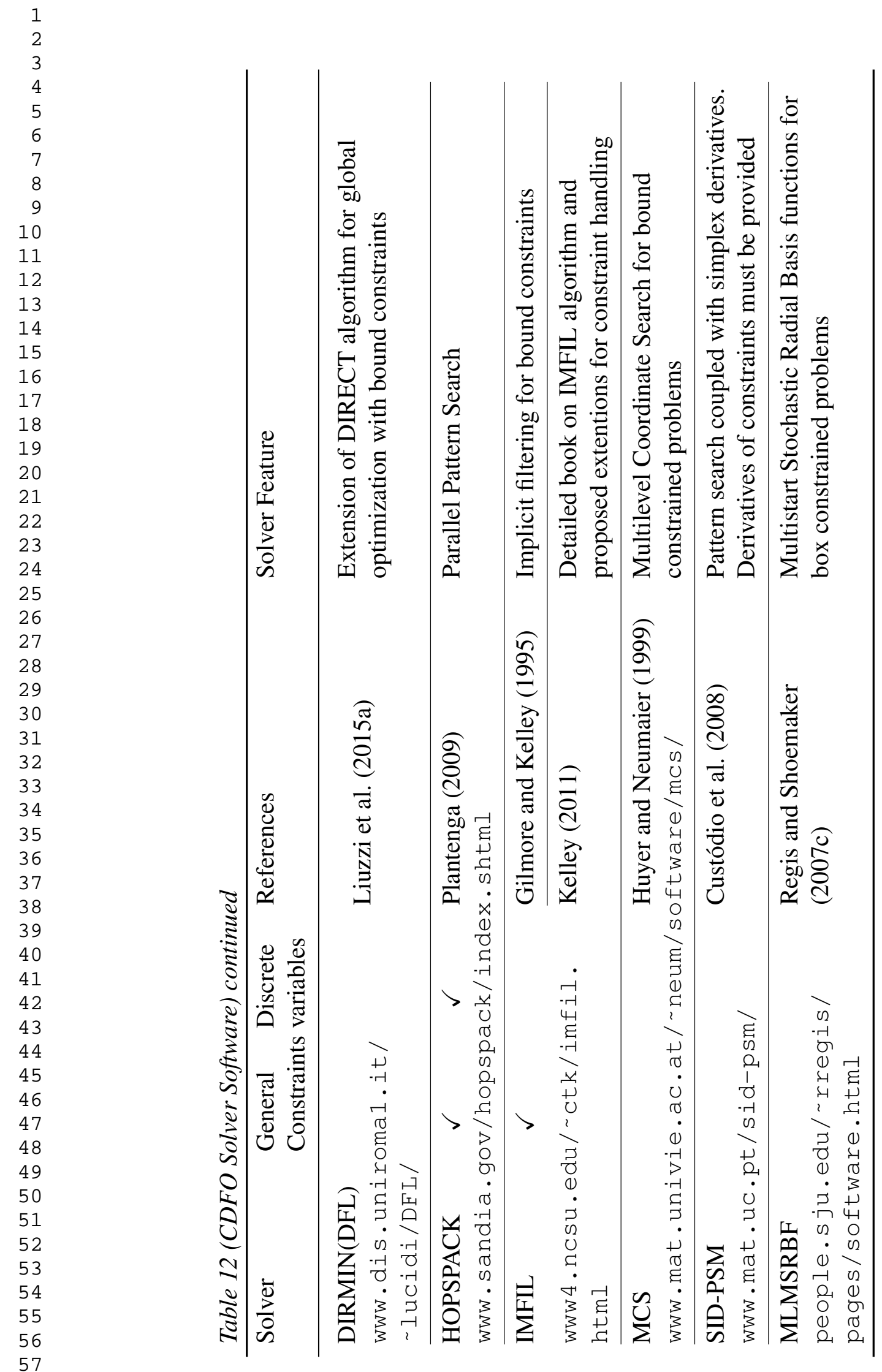




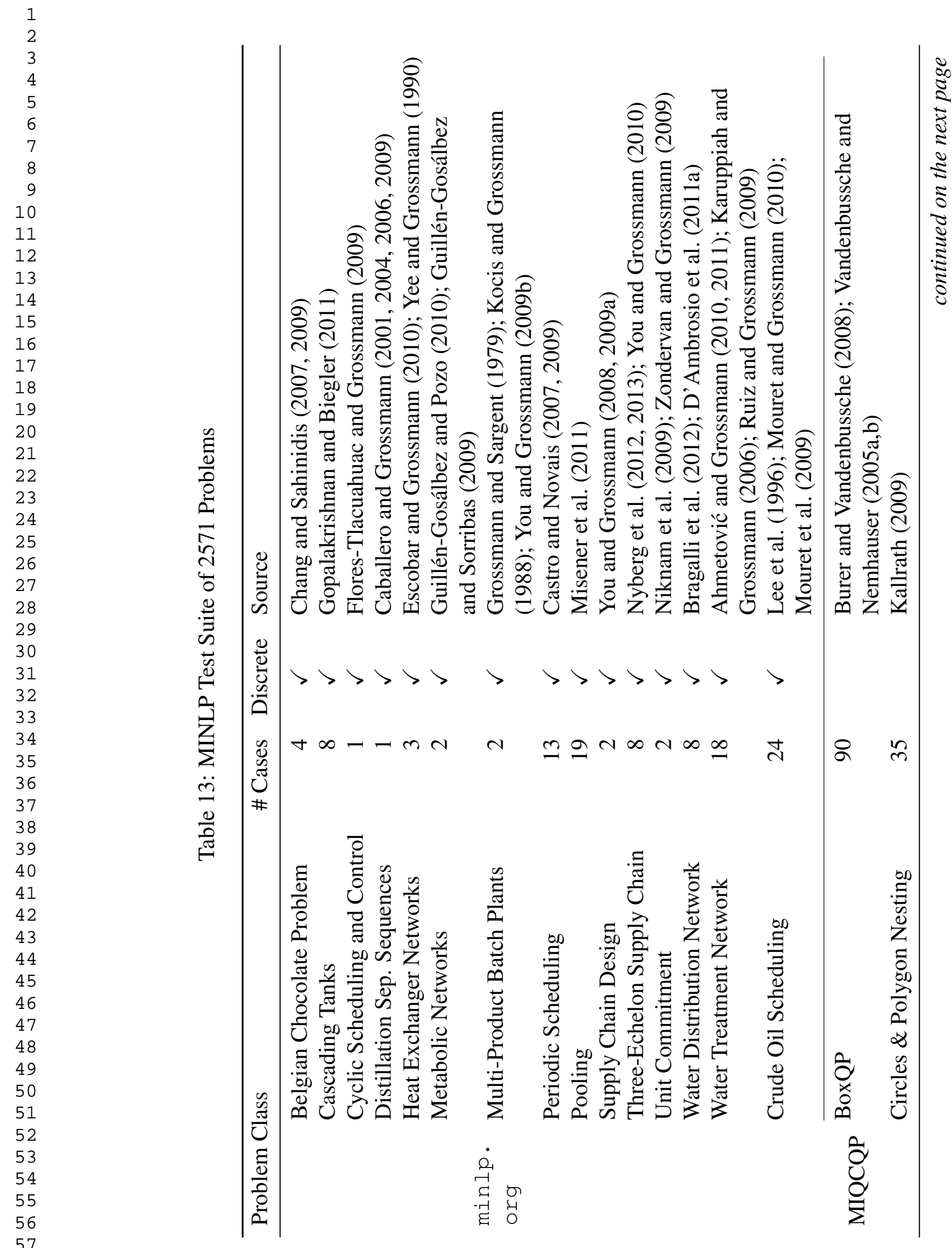









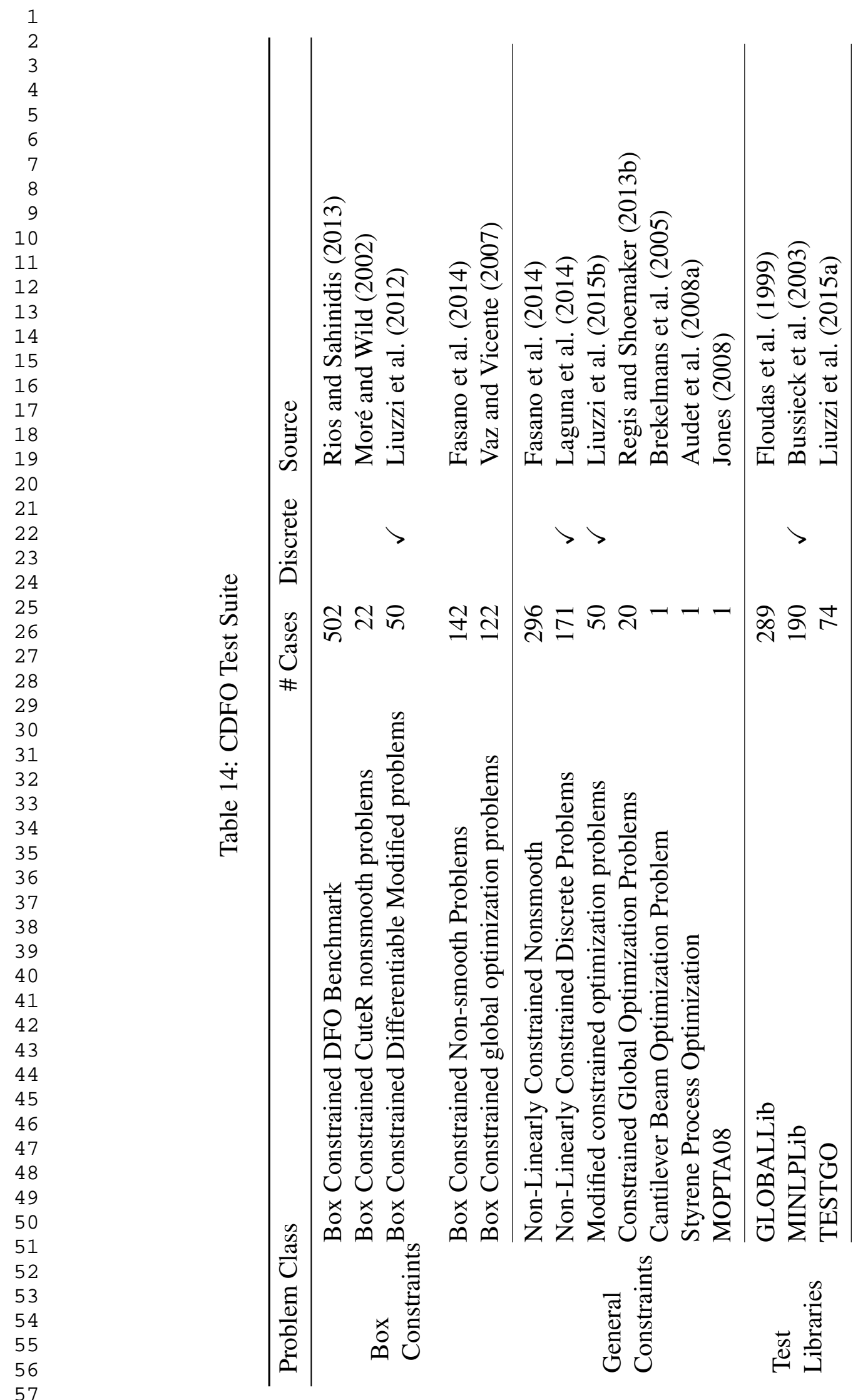




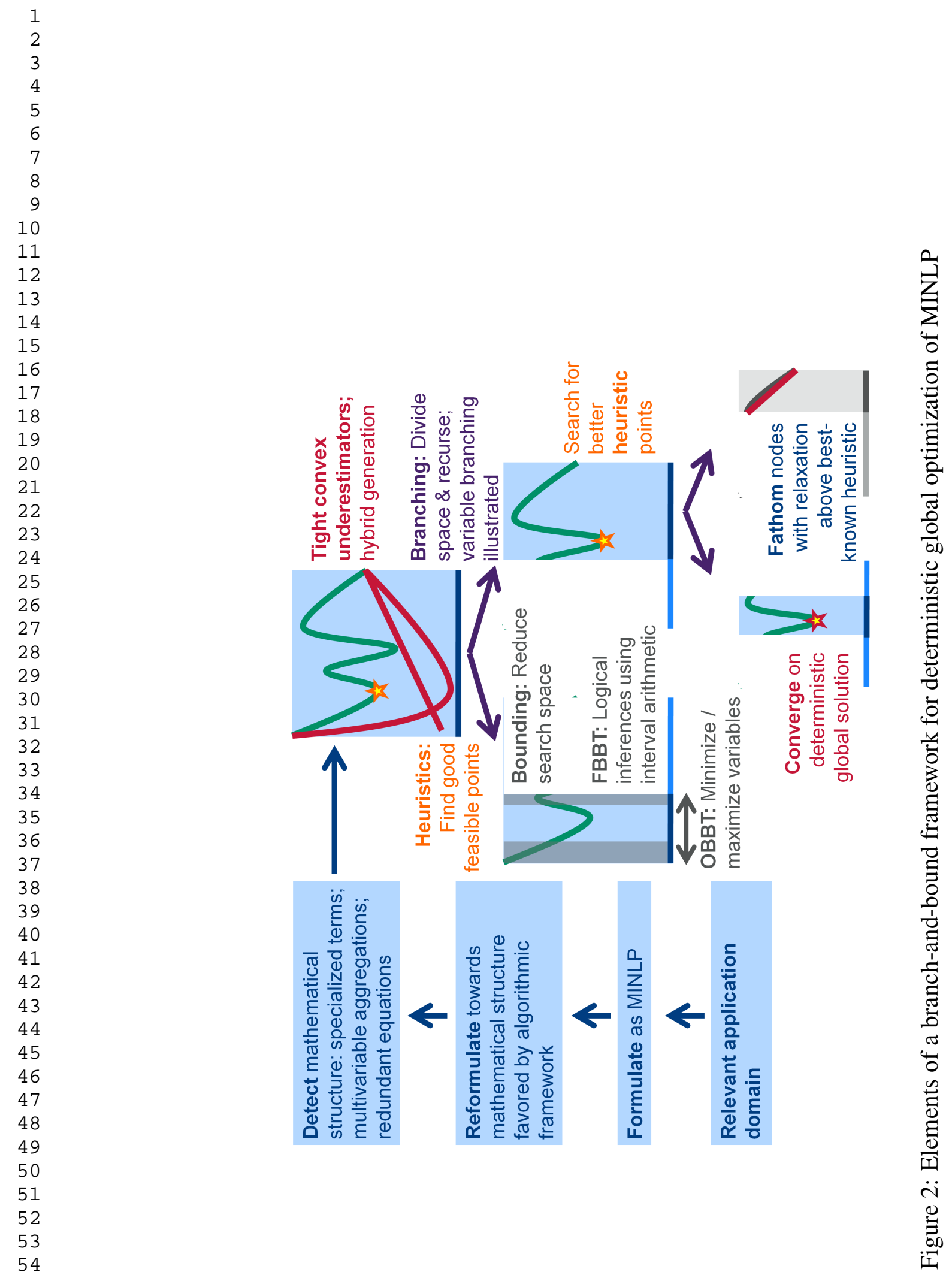

55

56

57

58

59

60

61

62

63

64

65 


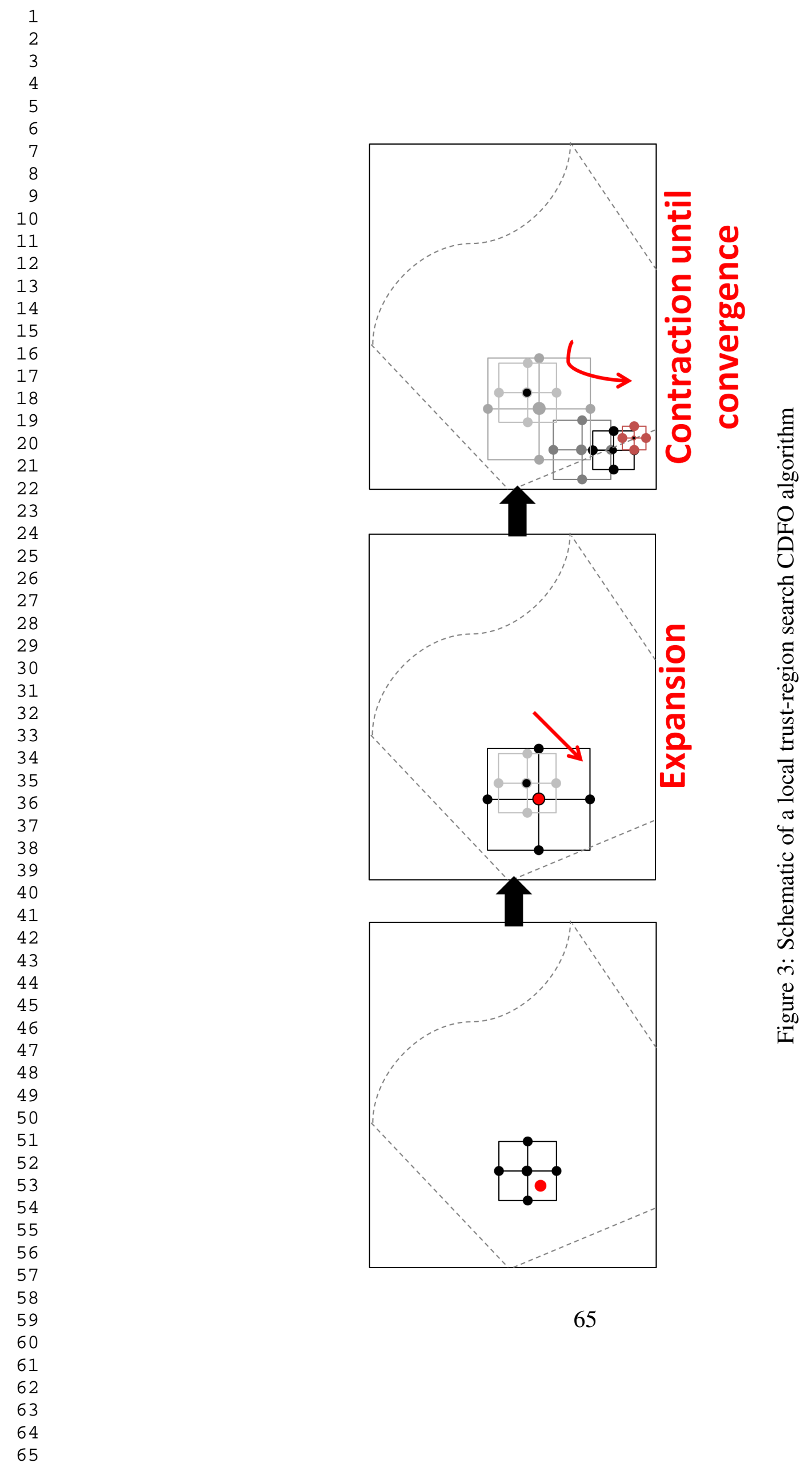




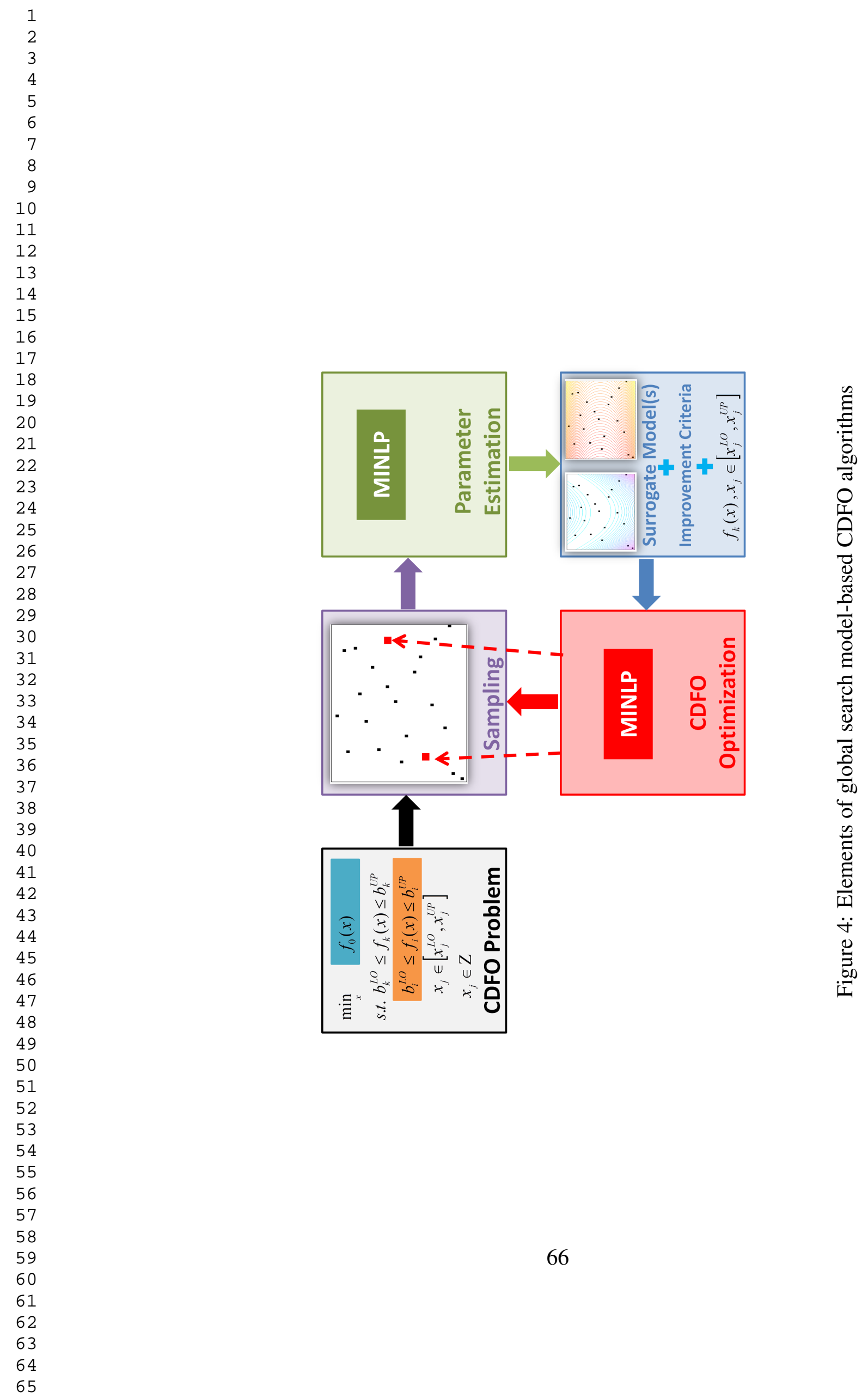




\section{References}

Abramson, M. A., 2002. Pattern search algorithms for mixed variable general constrained optimization problems. Ph.D. thesis, Ecole Polytechnique de Montreal.

Abramson, M. A., Asaki, T. J., Dennis, J. E., O’Reilly, K. R., Pingel, R. L., 2008. Quantitative object reconstruction using Abel transform X-Ray tomography and mixed variable optimization. SIAM J. Imaging Sci. 1 (3), 322-342.

Abramson, M. A., Asaki, T. J., Jr., J. E. D., Jr., R. M., Sottile, M. J., 2012. An efficient class of direct search surrogate methods for solving expensive optimization problems with cpu-time-related functions. Structural and Multidisciplinary Optimization 45 (1), 53-64.

Abramson, M. A., Audet, C., 2006. Convergence of mesh adaptive direct search to second-order stationary points. SIAM J. Optim. 17 (2), 606-619.

Abramson, M. A., Audet, C., Chrissis, J. W., Walston, J. G., 2009a. Mesh adaptive direct search algorithms for mixed variable optimization. Optim. Lett. 3 (1), $35-47$.

Abramson, M. A., Audet, C., Couture, G., Dennis, J. E., Le Digabel, S., Tribes, C., 2009b. The NOMAD project. Available at: http://www.gerad. ca/ nomad.

Abramson, M. A., Audet, C., Dennis, J. E., 2001. Filter pattern search algorithms for mixed variable constrained optimization problems. SIAM J. Optim. 11 (3), 573-594.

Abramson, M. A., Audet, C., Dennis, J. E., Le Digabel, S., 2009c. OrthoMADS: A deterministic MADS instance with orthogonal directions. SIAM J. Optim. 20 (2), 948-966.

Achterberg, T., 2009. SCIP: Solving constraint integer programs. Math. Program. Comput. 1 (1), 1-41.

Achterberg, T., Berthold, T., Koch, T., Wolter, K., 2008. Constraint integer programming: a new approach to integrate $\mathrm{CP}$ and MIP. In: Integration of AI and OR Techniques in Constraint Programming for Combinatorial Optimization Problems.

Achterberg, T., Koch, T., Martin, A., 2005. Branching rules revisited. Oper. Res. Lett. 33 (1), 42-54.

Adjengue, L., Audet, C., Yahia, I. B., 2013. A variance-based method to rank input variables of the mesh adaptive direct search algorithm. Optim. Lett., 112. 
Adjiman, C. S., Androulakis, I. P., Floudas, C. A., 1998a. A global optimization method, $\alpha \mathrm{BB}$, for general twice differentiable NLPs-II. Implementation and computional results. Comput. Chem. Eng. 22, $1159-1179$.

Adjiman, C. S., Androulakis, I. P., Floudas, C. A., 2000. Global optimization of mixed-integer nonlinear problems. AIChE J. 46 (9), 1769-1797.

Adjiman, C. S., Dallwig, S., Floudas, C. A., Neumaier, A., 1998b. A global optimization method, $\alpha \mathrm{BB}$, for general twice differentiable NLPs-I. Theoretical advances. Comput. Chem. Eng. 22, $1137-1158$.

Agarwal, A., Biegler, L. T., 2013. A trust-region framework for constrained optimization using reduced order modeling. Optim. Eng. 14 (1), 3-35.

Aggarwal, A., Floudas, C. A., 1990. Synthesis of general distillation sequences nonsharp separations. Comput. Chem. Eng. 14 (6), 631-653.

Ahmetović, E., Grossmann, I. E., 2010. Integrated process water networks design problem. Available from CyberInfrastructure for MINLP [A collaboration of CMU and IBM Research] at: www.minlp.org/library/problem/index.php? $i=101$.

Ahmetović, E., Grossmann, I. E., 2011. Global superstructure optimization for the design of integrated process water networks. AIChE J. 57 (2), 434-457.

Akrotirianakis, I. G., Floudas, C. A., 2004a. Computational experience with a new class of convex underestimators: Box-constrained NLP problems. J. Glob. Optim. 29 (3), 249-264.

Akrotirianakis, I. G., Floudas, C. A., 2004b. A new class of improved convex underestimators for twice continuously differentiable constrained NLPs. J. Glob. Optim. 30 (4), 367-390.

Akrotirianakis, I. G., Meyer, C. A., Floudas, C. A., 2004. The role of the offdiagonal elements of the Hessian matrix in the construction of tight convex underestimators for nonconvex functions. In: Discovery Through Product and Process Design. Foundations of Computer-Aided Process Design, pp. 501-504.

Al-Khayyal, F. A., Falk, J. E., 1983. Jointly constrained biconvex programming. Math. Oper. Res. 8 (2), 273 - 286.

Alfaki, M., Haugland, D., 2013. A multi-commodity flow formulation for the generalized pooling problem. J. Glob. Optim. 56 (3), 917-937.

Amaral, P., Bomze, I. M., Júdice, J., 2014. Copositivity and constrained fractional quadratic problems. Math. Program. 146 (1-2), 325-350.

Androulakis, I. P., Maranas, C. D., Floudas, C. A., 1995. $\alpha$ BB: A global optimization method for general constrained nonconvex problems. J. Glob. Optim. 
Anjos, M. F., Liers, F., 2012. Global approaches for facility layout and VLSI floorplanning. In: Anjos, M. F., Lasserre, J. B. (Eds.), Handbook on Semidefinite, Conic and Polynomial Optimization. Vol. 166 of International Series in Operations Research \& Management Science. Springer US, pp. 849-877.

Anstreicher, K. M., 2003. Recent advances in the solution of quadratic assignment problems. Math. Program. 97 (1-2), 27-42.

Anstreicher, K. M., 2009. Semidefinite programming versus the reformulationlinearization technique for nonconvex quadratically constrained quadratic programming. J. Glob. Optim. 43 (2-3), 471 - 484.

Anstreicher, K. M., Burer, S., 2010. Computable representations for convex hulls of low-dimensional quadratic forms. Math. Program. 124 (1-2), 33-43.

Arouxét, M. B., Echebest, N. E., Pilotta, E. A., 2015. Inexact restoration method for nonlinear optimization without derivatives. Journal of Computational and Applied Mathematics 290, 26 - 43.

Audet, C., Béchard, V., Chaouki, J., 2008a. Spent potliner treatment process optimization using a MADS algorithm. Optim. Eng. 9 (2), 143-160.

Audet, C., Béchard, V., Le Digabel, S., 2008b. Nonsmooth optimization through mesh adaptive direct search and variable neighborhood search. J. Glob. Optim. 41 (2), 299-318.

Audet, C., Brimberg, J., Hansen, P., Le Digabel, S., Mladenovic, N., 2004. Pooling problem: Alternate formulations and solution methods. Manage. Sci. 50 (6), $761-776$.

Audet, C., Dennis, J. E., 2001. Pattern search algorithms for mixed variable programming. SIAM J. Optim. 11 (3), 573-594.

Audet, C., Dennis, J. E., 2002. Analysis of generalized pattern searches. SIAM J. Optim. 13 (3), 889-903.

Audet, C., Dennis, J. E., 2004. A pattern search filter method for nonlinear programming without derivatives. SIAM J. Optim. 14 (4), 980-1010.

Audet, C., Dennis, J. E., 2006. Mesh adaptive direct search algorithms for constrained optimization. SIAM J. Optim. 17 (1), 188-217.

Audet, C., Dennis, J. E., 2009. A progressive barrier for derivative-free nonlinear programming. SIAM J. Optim. 20 (1), 445-472.

Audet, C., Dennis, J. E., Le Digabel, S., 2008c. Parallel space decomposition of the mesh adaptive direct search algorithm. SIAM J. Optim. 19 (3), 1150-1170. 
Audet, C., Dennis, J. E., Le Digabel, S., 2010. Globalization strategies formesh adaptive direct search. Comput. Optim. Appl. 46 (2), 193-215.

Audet, C., Guillou, A., Hansen, P., Messine, F., Perron, S., 2011. The small hexagon and heptagon with maximum sum of distances between vertices. J. Glob. Optim. 49 (3), 467-480.

Audet, C., Hansen, P., Jaumard, B., Savard, G., 2000. A branch and cut algorithm for nonconvex quadratically constrained quadratic programming. Math. Program. 87 (1), 131 - 152.

Audet, C., Hansen, P., Messine, F., 2007. The small octagon with longest perimeter. J. Combin. Theory Ser. A 114 (1), 135 - 150.

Audet, C., Hansen, P., Messine, F., 2009. Simple polygons of maximum perimeter contained inaunitdisk. Discrete Comput. Geom. 41 (2), 208-215.

Audet, C., Hansen, P., Messine, F., Xiong, J., 2002. The largest small octagon. J. Combin. Theory Ser. A 98 (1), $46-59$.

Audet, C., Ianni, I., Le Dibagel, S., Tribes, C., 2014. Reducing the number of function evaluations in mesh adaptive direct search algorithms. SIAM J. Optim. 24 (2), 621-642.

Audet, C., Ninin, J., 2013. Maximal perimeter, diameter and area of equilateral unit-width convex polygons. J. Glob. Optim. 56 (3), 1007-1016.

Bagajewicz, M., 2000. A review of recent design procedures for water networks in refineries and process plants. Comput. Chem. Eng. 24, 2093 - 2113.

Baliban, R. C., Elia, J. A., Floudas, C. A., 2013a. Biomass to liquid transportation fuels (BTL) systems: Process synthesis and global optimization framework. Energ. Environ. Sci. 6 (1), 267-287.

Baliban, R. C., Elia, J. A., Floudas, C. A., 2013b. Novel natural gas to liquids processes: Process synthesis and global optimization strategies. AIChE J. 59 (2), 505-531.

Baliban, R. C., Elia, J. A., Misener, R., Floudas, C. A., 2012a. Global optimization of a MINLP process synthesis model for thermochemical based conversion of hybrid coal, biomass, and natural gas to liquid fuels. Comput. Chem. Eng. 42, $64-86$.

Baliban, R. C., Elia, J. A., Weekman, V., Floudas, C. A., 2012b. Process synthesis of hybrid coal, biomass, and natural gas to liquids via Fischer-Tropsch synthesis, ZSM-5 catalytic conversion, methanol synthesis, methanol-to-gasoline, and methanol-to-olefins/distillate technologies. Comput. Chem. Eng. 47, 29 - 56.

Ballerstein, M., Michaels, D., 2014. Extended formulations for convex envelopes. 
Banga, J. R., Balsa-Canto, E., Moles, C. G., Alonso, A. A., 2005. Dynamic optimization of bioprocesses: Efficient and robust numerical strategies. J. Biotechnol. 117 (4), 407-419.

Banga, J. R., Irizarry-Rivera, R., Seider, W. D., 1998. Stochastic optimization for optimal and model-predictive control. Comput. Chem. Eng. 22 (4-5), 603-612.

Bao, X., Sahinidis, N. V., Tawarmalani, M., 2009. Multiterm polyhedral relaxations for nonconvex, quadratically-constrained quadratic programs. Optim. Method. Softw. 24 (4-5), 485 - 504.

Bao, X., Sahinidis, N. V., Tawarmalani, M., 2011. Semidefinite relaxations for quadratically constrained quadratic programming: A review and comparisons. Math. Program. 129 (1), 129-157.

Bartholomew-Biggs, M. C., Parkhurst, S. C., Wilson, S. P., 2002. Using DIRECT to solve an aircraft routing problem. Comput. Optim. Appl. 21 (3), 311-323.

Bartholomew-Biggs, M. C., Parkhurst, S. C., Wilson, S. P., 2003. Global optimization approaches to an aircraft routing problem. European Journal of Operational Research 146 (2), 417 - 431.

Belotti, P., 2013. Bound reduction using pairs of linear inequalities. J. Glob. Optim. 56 (3, SI), 787-819.

Belotti, P., Cafieri, S., Lee, J., Liberti, L., 2010a. Feasibility-based bounds tightening via fixed points. In: Wu, W., Daescu, O. (Eds.), Combinatorial Optimization and Applications. Vol. 6508 of Lecture Notes in Computer Science. Springer Berlin Heidelberg, pp. 65-76.

Belotti, P., Cafieri, S., Lee, J., Liberti, L., 2012. On feasibility based bounds tightening. http://www.optimization-online.org/DB_HTML/2012/ $01 / 3325 . h t m l$.

Belotti, P., Cafieri, S., Lee, J., Liberti, L., Miller, A., 2013a. On the composition of convex envelopes for quadrilinear terms. In: Chinchuluun, A., Pardalos, P. M., Enkhbat, R., Pistikopoulos, E. N. (Eds.), Optimization, Simulation, and Control. Vol. 76 of Springer Optimization and Its Applications. Springer New York, pp. 1-16.

Belotti, P., Kirches, C., Leyffer, S., Linderoth, J., Luedtke, J., Mahajan, A., 2013 b. Mixed-integer nonlinear optimization. Acta Numerica 22, 1-131.

Belotti, P., Lee, J., Liberti, L., Margot, F., Wächter, A., 2009. Branching and bounds tightening techniques for non-convex MINLP. Optim. Method. Softw. 24 (4-5), 597-634. 
Belotti, P., Miller, A. J., Namazifar, M., 2010b. Valid inequalities and convex hulls for multilinear functions. Electronic Notes in Discrete Mathematics 36, 805 812, ISCO 2010 - International Symposium on Combinatorial Optimization.

Ben-Tal, A., Eiger, G., Gershovitz, V., 1994. Global minimization by reducing the duality gap. Math. Program. 63, 193 - 212.

Benson, H. P., 2004. On the construction of convex and concave envelope formulas for bilinear and fractional functions on quadrilaterals. Comput. Optim. Appl. 27 (1), 5-22.

Bergamini, M. L., Grossmann, I., Scenna, N., Aguirre, P., 2008. An improved piecewise outer-approximation algorithm for the global optimization of MINLP models involving concave and bilinear terms. Comput. Chem. Eng. 32 (3), 477 $-493$.

Berthold, T., 2013. RENS - the optimal rounding. Math. Program. Comput. 6, 33-54.

Berthold, T., 2014. Heuristic algorithms in global MINLP solvers. PhD in Mathematics, Technischen Universität Berlin.

Berthold, T., Gleixner, A. M., 2013a. Undercover: a primal MINLP heuristic exploring a largest sub-MIP. Math. Program. 144, 315-346.

Berthold, T., Gleixner, A. M., 2013b. Undercover branching. In: Bonifaci, V., Demetrescu, C., Marchetti-Spaccamela, A. (Eds.), Experimental Algorithms. Vol. 7933 of Lecture Notes in Computer Science. Springer Berlin Heidelberg, pp. 212-223.

Berthold, T., Gleixner, A. M., Heinz, S., Vigerske, S., 2012a. Analyzing the computational impact of MIQCP solver components. Numerical Algebra, Control and Optimization 2 (4), $739-748$.

Berthold, T., Heinz, S., Vigerske, S., 2012b. Extending a CIP framework to solve MIQCPs. In: Lee, J., Leyffer, S. (Eds.), Mixed Integer Nonlinear Programming. Vol. 154 of The IMA Volumes in Mathematics and its Applications. Springer New York, pp. 427-444.

Biegler, L. T., Grossmann, I. E., 2004. Retrospective on optimization. Comput. Chem. Eng. 28 (8), 1169 - 1192.

Bienstock, D., Michalka, A., 2014. Cutting-planes for optimization of convex functions over nonconvex sets. SIAM J. Optim. 24 (2), 643-677.

Björkman, M., Holmström, K., 2000. Global optimization of costly nonconvex functions using radial basis functions. Optim. Eng. 1 (4), 373-397.

Bley, A., Gleixner, A. M., Koch, T., Vigerske, S., 2012. Comparing miqcp solvers 
to a specialised algorithm for mine production scheduling. In: Bock, H. G., Hoang, X. P., Rannacher, R., Schlöder, J. P. (Eds.), Modeling, Simulation and Optimization of Complex Processes. Springer Berlin Heidelberg, pp. 25-39.

Boland, N., Eberhard, A., Engineer, F., Tsoukalas, A., 2012. A new approach to the feasibility pump in mixed integer programming. SIAM J. Optim. 22 (3), 831-861.

Bompadre, A., Mitsos, A., 2011. Convergence rate of McCormick relaxations. J. Glob. Optim. 52 (1), 1-28.

Bomze, I. M., 2012. Copositive optimization: Recent developments and applications. Eur. J. Oper. Res. 216 (3), 509 - 520.

Bomze, I. M., Budinich, M., Pardalos, P. M., Pelillo, M., 1999. The maximum clique problem. In: Du, D.-Z., Pardalos, P. M. (Eds.), Handbook of Combinatorial Optimization. Springer US, pp. 1-74.

Bonami, P., Biegler, L. T., Conn, A. R., Cornuéjols, G., Grossmann, I. E., Laird, C. D., Lee, J., Lodi, A., Margot, F., Sawaya, N., Wächter, A., 2008. An algorithmic framework for convex mixed integer nonlinear programs. Discrete Optim. 5 (2), $186-204$.

Bonami, P., Olivares, A., Soler, M., Staffetti, E., 2013. Multiphase mixed-integer optimal control approach to aircraft trajectory optimization. J., Guid. Control Dynam. 36 (5), 1267 - 1277.

Bonilla-Petriciolet, A., Iglesias-Silva, G. A., Hall, K. R., 2009. Calculation of homogeneous azeotropes in reactive and non-reactive mixtures using a stochastic optimization approach. Fluid Phase Equilibr. 281 (1), 22 - 31.

Bonilla-Petriciolet, A., Rangaiah, G. P., Segovia-Hernandez, J. G., 2010. Evaluation of stochastic global optimization methods for modeling vapor-liquid equilibrium data. Fluid Phase Equilibr. 287 (2), 111 - 125.

Booker, A. J., Dennis, J. E., Frank, P. D., Serafini, D. B., Torczon, V., Trosset, M. W., 1999. A rigorous framework for optimization of expensive functions by surrogates. Struct. Multidiscip. Optim. 17 (1), 1-13.

Boukouvala, F., Hasan, M. M. F., Floudas, C. A., 2015. Argonaut: Algorithms for global optimization of constrained grey-box computational problemsAccepted for publication, Journal of Global Optimization.

Boukouvala, F., Ierapetritou, M. G., 2013. Surrogate-based optimization of expensive flowsheet modeling for continuous pharmaceutical manufacturing. J. Pharm. Innov. 8 (2), 131-145.

Boukouvala, F., Ierapetritou, M. G., 2014. Derivative-free optimization for ex- 
pensive constrained problems using a novel expected improvement objective function. AIChE J. 60 (7), 2462-2474.

Box, M. J., 1965. A new method of constrained optimization and a comparison with other methods. Comput. J. 8 (1), 42-52.

Bragalli, C., D'Ambrosio, C., Lee, J., Lodi, A., Toth, P., 2012. On the optimal design of water distribution networks: A practical MINLP approach. Optim. Eng. 13, 219-246.

Brekelmans, R., Driessen, L., Hamers, H., den Hertog, D., 2005. Constrained optimization involving expensive function evaluations: A sequential approach. Eur. J. Oper. Res. 160 (1), 121-138.

Brönnimann, H., Melquiond, G., Pion, S., 2003. The Boost interval arithmetic library. In: Proceedings of the 5th Conference on Real Numbers and Computers. Lyon, France, pp. 65-80.

Brönnimann, H., Melquiond, G., Pion, S., 2006. The design of the Boost interval arithmetic library. Theoretical Computer Science 351, 111-118.

Bruno, J. C., Fernandez, F., Castells, F., Grossmann, I. E., 1998. A rigorous MINLP model for the optimal synthesis and operation of utility plants. Chem. Eng. Res. Des. 76 (3), $246-258$.

Burer, S., Letchford, A. N., 2012. Non-convex mixed-integer nonlinear programming: A survey. Surveys in Operations Research and Management Science 17 (2), $97-106$.

Burer, S., Saxena, A., 2012. The MILP road to MIQCP. In: Lee, J., Leyffer, S. (Eds.), Mixed Integer Nonlinear Programming. Vol. 154 of The IMA Volumes in Mathematics and its Applications. Springer New York, pp. 373-405.

Burer, S., Vandenbussche, D., 2008. A finite branch-and-bound algorithm for nonconvex quadratic programming via semidefinite relaxations. Math. Program. 113 (2), 259-282.

Bussieck, M. R., Drud, A. S., Meeraus, A., 2003. MINLPLib - a collection of test models for mixed-integer nonlinear programming. INFORMS J. Comput. 15 (1), 114-119.

Bussieck, M. R., Vigerske, S., 2010. MINLP solver software. In: Cochran, J. J., Cox, L. A., Keskinocak, P., Kharoufeh, J. P., Smith, J. C. (Eds.), Wiley Encyclopedia of Operations Research and Management Science. John Wiley \& Sons, Inc.

Caballero, J. A., Grossmann, I. E., 2001. Generalized disjunctive programming model for the optimal synthesis of thermally linked distillation columns. Ind. 
Eng. Chem. Res. 40 (10), 2260-2274.

Caballero, J. A., Grossmann, I. E., 2004. Design of distillation sequences: From conventional to fully thermally coupled distillation systems. Comput. Chem. Eng. 28 (11), $2307-2329$.

Caballero, J. A., Grossmann, I. E., 2006. Structural considerations and modeling in the synthesis of heat integrated thermally coupled distillation sequences. Ind. Eng. Chem. Res. 45 (25), 8454-8474.

Caballero, J. A., Grossmann, I. E., 2008. An algorithm for the use of surrogate models in modular flowsheet optimization. AIChE J. 54 (10), 2633-2650.

Caballero, J. A., Grossmann, I. E., 2009. Optimal separation sequences based on distillation: From conventional to fully thermally coupled systems. Available from CyberInfrastructure for MINLP [A collaboration of CMU and IBM Research] at: www.minlp.org/library/problem/index.php?i=69.

Cafieri, S., Durand, N., 2014. Aircraft deconfliction with speed regulation: new models from mixed-integer optimization. J. Glob. Optim. 58 (4), 613-629.

Cafieri, S., Hansen, P., Létocart, L., Liberti, L., Messine, F., 2012. Compact relaxations for polynomial programming problems. In: Klasing, R. (Ed.), Experimental Algorithms. Vol. 7276 of Lecture Notes in Computer Science. Springer, Berlin, pp. 75-86.

Cafieri, S., Lee, J., Liberti, L., 2010. On convex relaxations of quadrilinear terms. J. Glob. Optim. 47, 661-685.

Cambini, R., Sodini, C., 2005. Decomposition methods for solving nonconvex quadratic programs via branch and bound. J. Glob. Optim. 33, 313-336.

Caprara, A., Locatelli, M., 2010. Global optimization problems and domain reduction strategies. Math. Program. 125 (1), 123-137.

Castillo, I., Westerlund, J., Emet, S., Westerlund, T., 2005. Optimization of block layout design problems with unequal areas: A comparison of MILP and MINLP optimization methods. Comput. Chem. Eng. 30 (1), 54 - 69.

Castillo, P. A. C., Mahalec, V., Kelly, J. D., 2013. Inventory pinch algorithm for gasoline blend planning. AIChE J. 59 (10), 3748-3766.

Castro, P., Novais, A., 2007. Optimal periodic scheduling of multistage continuous plants with single and multiple time grid formulations. Ind. Eng. Chem. Res. 46 (11), 3669-3683.

Castro, P., Novais, A., 2009. Periodic scheduling of continuous multiproduct plants. Available from CyberInfrastructure for MINLP [A collaboration of CMU and IBM Research] at: 
Castro, P. M., Matos, H. A., Novais, A. Q., 2007. An efficient heuristic procedure for the optimal design of wastewater treatment systems. Resources, Conservation and Recycling 50 (2), 158 - 185.

Castro, P. M., Teles, J. P., 2013. Comparison of global optimization algorithms for the design of water-using networks. Comput. Chem. Eng. 52, 249 - 261.

Castro, P. M., Teles, J. P., Novais, A. Q., 2009. Linear program-based algorithm for the optimal design of wastewater treatment systems. Clean Technol. Environ. Policy 11 (1), 83-93.

Chang, Y. J., Sahinidis, N. V., 2005. Optimization of metabolic pathways under stability considerations. Comput Chem Eng 29 (3), 467 - 479.

Chang, Y. J., Sahinidis, N. V., 2007. Global optimization in stabilizing controller design. J. Glob. Optim. 38, 509-526.

Chang, Y. J., Sahinidis, N. V., 2009. Stabilizing controller design and the Belgian chocolate problem. Available from CyberInfrastructure for MINLP [A collaboration of CMU and IBM Research] at: www.minlp.org/library/problem/index.php? $i=57$.

Ciric, A. R., Floudas, C. A., 1989. A retrofit approach for heat exchanger networks. Comput. Chem. Eng. 13 (6), 703 - 715.

Colmenares, T. R., Seider, W. D., 1989. Synthesis of utility systems integrated with chemical processes. Ind. Eng. Chem. Res. 28 (1), 84-93.

Conejo, P. D., Karas, E. W., Pedroso, L. G., Ribeiro, A. A., Sachine, M., 2013. Global convergence of trust-region algorithms for convex constrained minimization without derivatives. Applied Mathematics and Computation 220, 324 330.

Conn, A. R., Le Digabel, S., 2013. Use of quadratic models with mesh-adaptive direct search for constrained black box optimization. Optim. Method. Softw. 28 (1), 139-158.

Conn, A. R., Scheinberg, K., Vicente, L. N., 2009a. Global convergence of general derivative-free trust-region algorithms to first and second order critical points. SIAM J. Optim. 20, 387-415.

Conn, A. R., Scheinberg, K., Vicente, L. N., 2009b. Introduction to derivative-free optimization. MPS-SIAM Series on Optimization. SIAM, Philadelphia.

Csendes, T., Pal, L., Sendin, J. O. H., Banga, J. R., 2008. The GLOBAL optimization method revisited. Optim. Lett. 2 (4), 445-454.

Custódio, A., Madeira, J., 2015. Glods: Global and local optimization using direct 
Custódio, A. L., Dennis, J. E., Vicente, L. N., 2008. Using simplex gradients of nonsmooth functions in direct search methods. IMA Journal of Numerical Analysis 28 (4), 770-784.

Dahdah, T. H., Mitsos, A., 2014a. Structural optimization of seawater desalination: I. a flexible superstructure and novel MED-MSF configurations. Desalination $344,252-265$.

Dahdah, T. H., Mitsos, A., 2014b. Structural optimization of seawater desalination: II novel MED MSF TVC configurations. Desalination 344, 219 - 227.

Dalkiran, E., Sherali, H. D., 2013. Theoretical filtering of RLT bound-factor constraints for solving polynomial programming problems to global optimality. J. Glob. Optim. 57 (4), 1147-1172.

D’Ambrosio, C., Bragalli, C., Lee, J., Lodi, A., Toth, P., 2011a. Optimal design of water distribution networks. Available from CyberInfrastructure for MINLP [A collaboration of CMU and IBM Research] at: WWw. minlp.org/library/problem/index.php? $i=134$.

D’Ambrosio, C., Frangioni, A., Liberti, L., Lodi, A., 2010. On intervalsubgradient and no-good cuts. Oper. Res. Lett. 38 (5), 341 - 345.

D’Ambrosio, C., Frangioni, A., Liberti, L., Lodi, A., 2012. A storm of feasibility pumps for nonconvex MINLP. Math. Program. 136, 375-402.

D’Ambrosio, C., Linderoth, J., Luedtke, J., 2011b. Valid inequalities for the pooling problem with binary variables. In: Günlük, O., Woeginger, G. J. (Eds.), Integer Programming and Combinatoral Optimization. Vol. 6655 of Lecture Notes in Computer Science. Springer Berlin Heidelberg, pp. 117-129.

D’Ambrosio, C., Lodi, A., 2013. Mixed integer nonlinear programming tools: an updated practical overview. Annals of Operations Research 204, 301-320.

Das, S., Suganthan, P. N., 2011. Differential evolution: A survey of the state-ofthe-art. IEEE Trans. Evol. Comput. 15 (1), 4-31.

Davis, E., Ierapetritou, M. G., 2007. A kriging method for the solution of nonlinear programs with black-box functions. AIChE J. 53 (8), 2001-2012.

Davis, E., Ierapetritou, M. G., 2008. A kriging-based approach to MINLP containing black-box models and noise. Ind. Eng. Chem. Res. 47 (16), 6101-6125.

Davis, E., Ierapetritou, M. G., 2009. A kriging based method for the solution of mixed-integer nonlinear programs containing black-box functions. J. Glob. Optim. 43 (2-3), 191-205.

de Moura, L., Passmore, G. O., 2013. Computation in real closed infinitesimal 
and transcendental extensions of the rationals. In: Bonacina, M. P. (Ed.), Automated Deduction CADE-24. Vol. 7898 of Lecture Notes in Computer Science. Springer Berlin Heidelberg, pp. 178-192.

De Servi, C., Campanari, S., Tizzanini, A., Pietra, C., 2013. Enhancement of the electrical efficiency of commercial fuel cell units by means of an organic rankine cycle: a case study. J. Eng. Gas Turb. Power 135 (4), 042309.

Deng, Z., Bai, Y., Fang, S.-C., Tian, Y., Xing, W., 2013. A branch-and-cut approach to portfolio selection with marginal risk control in a linear conic programming framework. J. Sys. Sci. Sys. Eng. 22 (4), 385-400.

Di Pillo, G., Lucidi, S., Rinaldi, F., 2013. A derivative-free algorithm for constrained global optimization based on exact penalty functions. J. Optim. Theory Appl.DOI 10.1007/s10957-013-0487-1.

di Serafino, D., Gomez, S., Milano, L., Riccio, F., Toraldo, G., 2010. A genetic algorithm for a global optimization problem arising in the detection of gravitational waves. J. Glob. Optim. 48 (1), 41-55.

Ding, Y., Ge, D., Wolkowicz, H., 2011. On equivalence of semidefinite relaxations for quadratic matrix programming. Math. Oper. Res. 36 (1), 88-104.

Dipama, J., Teyssedou, A., Sorin, M., 2008. Synthesis of heat exchanger networks using genetic algorithms. Appl. Therm. Eng. 28 (14-15), 1763 - 1773.

Domes, F., 2009. GloptLab - a configurable framework for the rigorous global solution of quadratic constraint satisfaction problems. Optim. Method. Softw. $24,727-747$.

Domes, F., Neumaier, A., 2010. Constraint propagation on quadratic constraints. Constraints 15 (3), 404-429.

Domes, F., Neumaier, A., 2011. Rigorous enclosures of ellipsoids and directed cholesky factorizations. SIAM J. Matrix Anal. A. 32, 262-285.

Domes, F., Neumaier, A., 2014. Constraint aggregation for rigorous global optimization. Mathematical ProgrammingDOI 10.1007/s10107-014-0851-4.

Domschke, P., Geissler, B., Kolb, O., Lang, J., Martin, A., Morsi, A., 2011. Combination of nonlinear and linear optimization of transient gas networks. INFORMS J. Comput. 23 (4), 605-617.

Dorneich, M. C., Sahinidis, N. V., 1995. Global optimization algorithms for chip layout and compaction. Eng. Optim. 25, 131-154.

Du, K., Kearfott, R. B., 1994. The cluster problem in multivariate global optimization. J. Glob. Optim. 5 (3), 253-265.

Duran, M. A., Grossmann, I. E., 1986a. A mixed-integer nonlinear programming 
Duran, M. A., Grossmann, I. E., 1986b. An outer-approximation algorithm for a class of mixed-integer nonlinear programs. Math. Program. 36, 307-339.

Egea, J. A., Martí, R., Banga, J. R., 2010. An evolutionary method for complexprocess optimization. Comput. Oper. Res. 37 (2), 315-324.

Egea, J. A., Rodrigues-Fernandez, M. P., Banga, J. R., Marti, R., 2007a. Scatter search for chemical and bio-process optimization. J. Glob. Optim. 37 (3), 481503.

Egea, J. A., Vries, D., Alonso, A. A., Banga, J. R., 2007b. Global optimization for integrated design and control of computationally expensive process models. Ind. Eng. Chem. Res. 46 (26), 9148-9157.

Eldred, M. S., Adams, B. M., Gay, D. M., Swiller, L. P., Haskell, K., Bohnhoff, W. J., Eddy, J. P., Hart, W. E., Watson, J. P., Hough, P. D., Kolda, T. G., 2008. DAKOTA, a multilevel parallel object-oriented framework for design optimization, parameter estimation, uncertainty quantification and sensitivity analysis. Available at: http://dakota.sandia.gov/docs/dakota/ 4.2/Developers 4.2.pdf.

Engelhart, M., Funke, J., Sager, S., 2013. A decomposition approach for a new test-scenario in complex problem solving. J. Comput. Sci. 4 (4), 245 - 254.

Escobar, M., Grossmann, I. E., 2010. Mixed-integer nonlinear programming models for optimal simultaneous synthesis of heat exchangers network. Available from CyberInfrastructure for MINLP [A collaboration of CMU and IBM Research] at: www.minlp.org/library/problem/index.php?i=93.

Espinet, A., Shoemaker, C., Doughty, C., 2013. Estimation of plume distribution for carbon sequestration using parameter estimation with limited monitoring data. Water Resources Research 49 (7), 4442-4464.

Fahmi, I., Cremaschi, S., 2012. Process synthesis of biodiesel production plant using artificial neural networks as the surrogate models. Comput. Chem. Eng. 46, 105-123.

Faria, D. C., Bagajewicz, M. J., 2012. A new approach for global optimization of a class of minlp problems with applications to water management and pooling problems. AIChE J. 58 (8), 2320-2335.

Fasano, G., Liuzzi, G., Lucidi, S., Rinaldi, F., 2014. A linesearch-based derivativefree approach for nonsmooth constrained optimization. SIAM J. Optim. 24 (3), 959-992.

First, E. L., Hasan, M. M. F., Floudas, C. A., 2014. Discovery of novel zeolites 
for natural gas purification through combined material screening and process optimization. AIChE J. 60 (5), 1767-1785.

Flores-Tlacuahuac, A., Grossmann, I. E., 2009. Simultaneous cyclic scheduling and control of a multiproduct CSTR. Available from CyberInfrastructure for MINLP [A collaboration of CMU and IBM Research] at: WWw. minlp.org/library/problem/index.php? $i=71$.

Floudas, C. A., 1995. Nonlinear and Mixed-Integer Optimization: Fundamentals and Applications. Oxford University Press, New York, NY.

Floudas, C. A., 2000. Deterministic Global Optimization : Theory, Methods and Applications. Nonconvex Optimization and Its Applications. Kluwer Academic Publishers, Dordrecht, Netherlands.

Floudas, C. A., Aggarwal, A., 1990. A decomposition strategy for global optimum search in the pooling problem. ORSA J. Comput. 2, 225 - 235.

Floudas, C. A., Akrotirianakis, I. G., Caratzoulas, S., Meyer, C. A., Kallrath, J., 2005. Global optimization in the 21st century: Advances and challenges. Comput. Chem. Eng. 29, 1185 - 1202.

Floudas, C. A., Anastasiadis, S. H., 1988. Synthesis of distillation sequences with several multicomponent feed and product streams. Chem. Eng. Sci. 43 (9), 2407-2419.

Floudas, C. A., Elia, J. A., Baliban, R. C., 2012. Hybrid and single feedstock energy processes for liquid transportation fuels: A critical review. Comput. Chem. Eng. 41, $24-51$.

Floudas, C. A., Gounaris, C. E., 2009. A review of recent advances in global optimization. J. Glob. Optim. 45 (1), 3 - 38.

Floudas, C. A., Grossmann, I. E., 1987. Synthesis of flexible heat-exchanger networks with uncertain flowrates and temperatures. Comput. Chem. Eng. 11 (4), 319-336.

Floudas, C. A., Pardalos, P. M. (Eds.), 1992. Recent Advances In Global Optimization. Princeton University Press, Princeton, New Jersey.

Floudas, C. A., Pardalos, P. M., 1995. State-of-the-art in global optimization computational methods and applications - Preface. J. Glob. Optim. 7 (2), 113.

Floudas, C. A., Pardalos, P. M. (Eds.), 1996. State of the Art In Global Optimization: Computational Methods and Applications. Kluwer Academic Publishers.

Floudas, C. A., Pardalos, P. M. (Eds.), 2000. Optimization in Computational Chemistry and Molecular Biology: Local and Global Approaches. Nonconvex Optimization and Its Applications. Kluwer Academic Publishers. 
Floudas, C. A., Pardalos, P. M. (Eds.), 2004. Frontiers in Global Optimization. Nonconvex Optimization and Its Applications, Kluwer Academic Publishers.

Floudas, C. A., Pardalos, P. M., Adjiman, C. S., Esposito, W. R., Gümüs, Z. H., Harding, S. T., Klepeis, J. L., Meyer, C. A., Schweiger, C. A., 1999. Handbook of Test Problems in Local and Global Optimization. Kluwer Academic Publishers.

Floudas, C. A., Paules, G. E., 1988. A mixed-integer nonlinear programming formulation for the synthesis of heat-integrated distillation sequences. Comput. Chem. Eng. 12 (6), 531 - 546.

Floudas, C. A., Visweswaran, V., 1990. A global optimization algorithm (GOP) for certain classes of nonconvex NLPs: I. Theory. Comput. Chem. Eng. 14 (12), 1397 - 1417.

Floudas, C. A., Visweswaran, V., 1993. Primal-relaxed dual global optimization approach. J. Optim. Theory Appl. 78 (2), 187 - 225.

Forrester, A. I. J., Jones, D. R., 2008. Global optimization of deceptive functions with sparse sampling. In: AIAA/ISSMO Multidisciplinary Analysis and Optimization Conference. American Institute of Aeronautics and Astronautics, Victoria, British Columbia Canada.

Forrester, A. I. J., Keane, A. J., 2009. Recent advances in surrogate-based optimization. Progress in Aerospace Sciences 45 (1), 50-79, 0376-0421.

Forrester, A. I. J., Sobester, A., Keane, A. J., 2008. Engineering Design via Surrogate Modelling - A Practical Guide. John Wiley \& Sons.

Fourer, R., Gay, D. M., Kernighan, B. W., 2002. The AMPL Book. AMPL: A Modeling Language for Mathematical Programming. Duxbury Press, Brooks/Cole Publishing Company.

Fowler, K. R., Reese, J. P., Kees, C. E., Dennis, J. E., Kelley, C. T., 2008. Comparison of derivative-free optimization methods for groundwater supply and hydraulic capture community problems. Water Resources 31, 743-757.

Fügenschuh, A., Geissler, B., Gollmer, R., Hayn, C., Henrion, R., Hiller, B., Humpola, J., Koch, T., Lehmann, T., Martin, A., Mirkov, R., Morsi, A., Rövekamp, J., Schewe, L., Schmidt, M., Schultz, R., Schwarz, R., Schweiger, J., Stangl, C., Steinbach, M. C., Willert, B. M., 2014. Mathematical optimization for challenging network planning problems in unbundled liberalized gas markets. Energy Systems 5 (3), 449-473.

Fügenschuh, A., Homfeld, H., Schülldorf, H., Vigerske, S., 2010. Mixed-integer nonlinear problems in transportation applications. In: Rodrigues, H. (Ed.), Pro- 
ceedings of the 2nd International Conference on Engineering Optimization.

Gablonsky, J. M., 2001. Modifications of the DIRECT algorithm. PhD in Mathematics, North Carolina State University.

Garcia-Palomares, U. M., Costa-Montenegro, E., Asorey-Cacheda, R., GonzalezCastano, F. J., 2012. Adapting derivative free optimization methods to engineering models with discrete variables. Optimization and Engineering 13 (4), 579-594.

Garcia-Palomares, U. M., Garcia-Urrea, I. J., Rodriguez-Hernandez, P. S., 2013. On sequential and parallel non-monotone derivative-free algorithms for box constrained optimization. Optim. Method. Softw. 28 (6), 1233-1261.

Garcia-Palomares, U. M., Gonzalez-Castano, F. J., Burguillo-Rial, J. C., 2006. A combined global and local search (CGLS) approach to global optimization. J. Glob. Optim. 34 (3), 409-426.

Gassner, M., Maréchal, F., 2009. Methodology for the optimal thermo-economic, multi-objective design of thermochemical fuel production from biomass. Comput. Chem. Eng. 33 (3), 769 - 781.

Gatzke, E. P., Tolsma, J. E., Barton, P. I., 2002. Construction of convex relaxations using automated code generation techniques. Optim. Eng. 3, 305-326.

Gau, C. Y., Schrage, L. E., 2003. Implementation and testing of a branch-andbound based method for deterministic global optimization: Operations research applications. In: Floudas, C. A., Pardalos, P. M. (Eds.), Frontiers in Global Optimization. Kluwer Academic Publishers, pp. 145-164.

Geissler, B., Morsi, A., Schewe, L., 2013. A new algorithm for MINLP applied to gas transport energy cost minimization. In: Jünger, M., Reinelt, G. (Eds.), Facets of Combinatorial Optimization. Springer Berlin Heidelberg, pp. 321353.

Gentilini, I., Margot, F., Shimada, K., 2013. The travelling salesman problem with neighbourhoods: MINLP solution. Optim Met Softw 28 (2), 364-378.

Geoffrion, A. M., 1970. Elements of Large-Scale Mathematical Programming .1. Concepts. Management Science Series A-Theory 16 (11), 652-675.

Gilmore, P., Kelley, C., 1995. An implicit filtering algorithm for optimization of functions with many local minima. SIAM J. Optim. 5 (2), 269-285.

Gleixner, A. M., Held, H., Huang, W., Vigerske, S., 2012. Towards globally optimal operation of water supply networks. Numerical Algebra, Control and Optimization 2 (4), 695-711.

Gleixner, A. M., Weltge, S., 2013. Learning and propagating Lagrangian variable 
bounds for mixed-integer nonlinear programming. In: Gomes, C., Sellmann, M. (Eds.), Integration of AI and OR Techniques in Constraint Programming for Combinatorial Optimization Problems. Vol. 7874 of Lecture Notes in Computer Science. Springer Berlin Heidelberg, pp. 355-361.

Gopalakrishnan, A., Biegler, L., 2011. MINLP and MPCC formulations for the cascading tanks problem. Available from CyberInfrastructure for MINLP [A collaboration of CMU and IBM Research] at: WwW.minlp.org/library/problem/index.php? $i=140$.

Gounaris, C. E., First, E. L., Floudas, C. A., 2013. Estimation of diffusion anisotropy in microporous crystalline materials and optimization of crystal orientation in membranes. J. Chem. Phys. 139 (12), 124703.

Gounaris, C. E., Floudas, C. A., 2008a. Convexity of products of univariate functions and convexification transformations for geometric programming. J. Optim. Theory Appl. 138, 407-427.

Gounaris, C. E., Floudas, C. A., 2008b. Tight convex underestimators for $\mathscr{C}^{2}$ continuous problems: I. Univariate functions. J. Glob. Optim. 42 (1), 51-67.

Gounaris, C. E., Floudas, C. A., 2008c. Tight convex underestimators for $\mathscr{C}^{2}$ continuous problems: II. Multivariate functions. J. Glob. Optim. 42 (1), 69-89.

Gounaris, C. E., Misener, R., Floudas, C. A., 2009. Computational comparison of piecewise-linear relaxations for pooling problems. Ind. Eng. Chem. Res. 48 (12), 5742 - 5766.

Graciano, J. E. A., Roux, G. A. C. L., 2013. Improvements in surrogate models for process synthesis. application to water network system design. Comput. Chem. Eng. 59, $197-210$.

Granvilliers, L., Benhamou, F., 2006. Algorithm 852: RealPaver: An interval solver using constraint satisfaction techniques. ACM Trans. Math. Softw. 32 (1), 138-156.

Gratton, S., Toint, P., Tröltzsch, A., 2011. An active-set trust-region method for derivative-free nonlinear bound-constrained optimization. Optim. Method. Softw. 26 (4-5), 873-894.

Gratton, S., Vicente, L., 2014. A merit function approach for direct search. SIAM Journal on Optimization 24 (4), 1980-1998.

Gray, G. A., Kolda, T. G., Sale, K., Young, M. M., 2004. Optimizing an empirical scoring function for transmembrane protein structure determination. INFORMS J. Comput. 16 (4), 406-418.

Griffin, J. D., Kolda, T. G., 2010. Asynchronous parallel hybrid optimization com- 
Gross, B., Roosen, P., 1998. Total process optimization in chemical engineering with evolutionary algorithms. Comput. Chem. Eng. 22 (1), S229 - S236.

Grossmann, I. E., 2012. Advances in mathematical programming models for enterprise-wide optimization. Comput. Chem. Eng. 47, 2 - 18.

Grossmann, I. E., Biegler, L. T., 2004. Part II. Future perspective on optimization. Comput. Chem. Eng. 28 (8), 1193 - 1218.

Grossmann, I. E., Guillén-Gosálbez, G., 2010. Scope for the application of mathematical programming techniques in the synthesis and planning of sustainable processes. Comput. Chem. Eng. 34 (9), 1365 - 1376.

Grossmann, I. E., Sargent, R. W. H., 1979. Optimum design of multipurpose chemical plants. Ind. Eng. Chem. Process Des. Dev. 18 (2), 343-348.

Guillén-Gosálbez, G., Pozo, C., 2010. Optimization of metabolic networks in biotechnology. Available from CyberInfrastructure for MINLP [A collaboration of CMU and IBM Research] at: www. minlp.org/library/problem/index.php? $i=81$.

Guillén-Gosálbez, G., Sorribas, A., 2009. Identifying quantitative operation principles in metabolic pathways: a systematic method for searching feasible enzyme activity patterns leading to cellular adaptive responses. BMC Bioinformatics 10, 386.

Gupte, A., Ahmed, S., Cheon, M., Dey, S., 2013. Solving mixed integer bilinear problems using MILP formulations. SIAM J. Opt. 23 (2), 721-744.

Guzman, Y. A., Hasan, M. M. F., Floudas, C. A., 2014. Computational comparison of convex underestimators for use in a branch-and-bound global optimization framework. In: Rassias, T. M., Floudas, C. A., Butenko, S. (Eds.), Optimization in Science and Engineering. Springer New York, pp. 229-246.

Hager, W., Phan, D., 2009. An ellipsoidal branch and bound algorithm for global optimization. SIAM J. Opt. 20 (2), 740-758.

Han, J., Kokkolaras, M., Papalambros, P. Y., 2008. Optimal design of hybrid fuel cell vehicles. Journal of Fuel Cell Science and Technology 5 (4).

Hare, W., Nutini, J., 2013. A derivative-free approximate gradient sampling algorithm for finite minimax problems. Comput. Optim. Appl. 56 (1), 1-38.

Harjunkoski, I., Maravelias, C. T., Bongers, P., Castro, P. M., Engell, S., Grossmann, I. E., Hooker, J., Méndez, C., Sand, G., Wassick, J., 2014. Scope for industrial applications of production scheduling models and solution methods. Comput. Chem. Eng. 62, 161 - 193. 
Harjunkoski, I., Westerlund, T., Isaksson, J., Skrifvars, H., 1996. Different formulations for solving trim loss problems in a paper-converting mill with ILP. Comput. Chem. Eng. 20, Supplement 1, S121 - S126.

Harjunkoski, I., Westerlund, T., Pörn, R., Skrifvars, H., 1998. Different transformations for solving non-convex trim-loss problems by MINLP. Eur. J. Oper. Res. 105 (3), 594 - 603.

Hasan, M. M. F., Baliban, R. C., Elia, J. A., Floudas, C. A., 2012. Modeling, simulation, and optimization of postcombustion $\mathrm{CO} 2$ capture for variable feed concentration and flow rate. 2. pressure swing adsorption and vacuum swing adsorption processes. Ind. Eng. Chem. Res. 51 (48), 15665-15682.

Hasan, M. M. F., Boukouvala, F., First, E. L., Floudas, C. A., 2014. Nationwide, regional, and statewide $\mathrm{CO} 2$ capture, utilization, and sequestration supply chain network optimization. Ind. Eng. Chem. Res. 53 (18), 7489-7506.

Hasan, M. M. F., First, E. L., Boukouvala, F., Floudas, C. A., 2015. A multi-scale framework for $\mathrm{CO} 2$ capture, utilization and sequestration: CCUS and CCU. Computers and Chemical Engineering 81, 2-21.

Hasan, M. M. F., First, E. L., Floudas, C. A., 2013. Cost-effective CO2 capture based on in silico screening of zeolites and process optimization. Phys. Chem. Chem. Phys. 15, 17601-17618.

Hasan, M. M. F., Karimi, I. A., 2010. Piecewise linear relaxation of bilinear programs using bivariate partitioning. AIChE J. 56 (7), 1880 - 1893.

Hasan, M. M. F., Karimi, I. A., Avison, C. M., 2011. Preliminary synthesis of fuel gas networks to conserve energy and preserve the environment. Ind. Eng. Chem. Res. 50 (12), 7414-7427.

Hayes, R. E., Bertrand, F. H., Audet, C., Kolaczkowski, S. T., 2003. Catalytic combustion kinetics: Using a direct search algorithm to evaluate kinetic parameters from light-off curves. The Canadian Journal of Chemical Engineering 81 (6), 1192-1199.

Hemker, T., Werner, C., 2011. DIRECT using local search on surrogates. Pacific Journal of Optimization 7 (3), 443-466.

Hemmecke, R., Köppe, M., Lee, J., Weismantel, R., 2010. Nonlinear integer programming. In: Jünger, M., Liebling, T. M., Naddef, D., Nemhauser, G. L., Pulleyblank, W. R., Reinelt, G., Rinaldi, G., Wolsey, L. A. (Eds.), 50 Years of Integer Programming 1958-2008. Springer Berlin Heidelberg, pp. 561-618.

Henao, C. A., Maravelias, C. T., 2011. Surrogate-based superstructure optimization framework. AIChE J. 57 (5), 1216-1232. 
Henrion, D., Lasserre, J.-B., 2003. GloptiPoly: Global optimization over polynomials with Matlab and SeDuMi. ACM Trans. Math. Softw. 29 (2), 165-194.

Henrion, D., Lasserre, J. B., Löfberg, J., 2009. GloptiPoly 3: Moments, optimization and semidefinite programming. Optim. Methods Soft. 24 (4-5), 761-779.

Hifi, M., M'Hallah, R., 2009. A literature review on circle and sphere packing problems: Models and methodologies. Advances in Operations Research 2009, 22.

Hladík, M., 2014. On the efficient Gerschgorin inclusion usage in the global optimization $\alpha$ BB method. J. Glob. Optim.DOI: 10.1007/s10898-014-0161-7.

Hoai An, L. T., 2000. An efficient algorithm for globally minimizing a quadratic function under convex quadratic constraints. Math. Program. 87, 401-426.

Holmström, K., Göran, A. O., Edvall, M. M., 2008a. Users guide for TOMLAB CGO. Available at: http://tomopt.com/docs/TOMLAB_CGO.pdf.

Holmström, K., Quttineh, N.-H., Edvall, M. M., 2008b. An adaptive radial basis algorithm (ARBF) for expensive black-box mixed-integer constrained global optimization. Optim. Eng. 9 (4), 311-339.

Hooke, R., Jeeves, T. A., 1961. Direct search solution of numerical and statistical problems. J. ACM 8 (2), 212-229.

Horst, R., Tuy, H., 1996. Global optimization: Deterministic approaches. Springer.

Huyer, W., Neumaier, A., 1999. Global optimization by multilevel coordinate search. J. Glob. Optim. 14 (4), 331-355.

Huyer, W., Neumaier, A., 2008. SNOBFIT - stable noisy optimization by branch and fit. ACM Transactions on Mathematical Software 35 (2).

Jach, M., Michaels, D., Weismantel, R., 2008. The convex envelope of (n-1) convex functions. SIAM J. Optim. 19 (3), 1451-1466.

Jakobsson, S., Patriksson, M., Rudholm, J., Wojciechowski, A., 2010. A method for simulation based optimization using radial basis functions. Optim. Eng. 11 (4), 501-532.

Jansson, N., Wakeman, W. D., Manson, J. A. E., 2007. Optimization of hybrid thermoplastic composite structures using surrogate models and genetic algorithms. Composite Structures 80 (1), 21-31.

Jeżowski, J., 2010. Review of water network design methods with literature annotations. Ind. Eng. Chem. Res. 49 (10), 4475 - 4516.

Jones, D. R., 2001. A taxonomy of global optimization methods based on response 
Jones, D. R., 2008. MOPTA 2008 benchmark. Available at: http: / / an jos . mgi.polymtl.ca/MOP TA2008Benchmark.html.

Jones, D. R., Perttunen, C. D., Stuckman, B. E., 1993. Lipschitzian optimization without the Lipschitz constant. J. Optim. Theory Appl. 79 (1), 157-181.

Jones, D. R., Schonlau, M., Welch, W. J., 1998. Efficient global optimization of expensive black-box functions. J. Glob. Optim. 13 (4), 455-492.

Kallrath, J., 2003. Exact computation of global minima of a nonconvex portfolio optimization problem. In: Floudas, C. A., Pardalos, P. M. (Eds.), Frontiers in Global Optimization. Kluwer Academic Publishers, pp. 237-254.

Kallrath, J., 2009. Cutting circles and polygons from area-minimizing rectangles. J. Glob. Optim. 43, 299 - 328.

Kallrath, J., Rebennack, S., 2014. Cutting ellipses from area-minimizing rectangles. J. Glob. Optim. 59 (2-3), 405-437.

Karuppiah, R., Grossmann, I. E., 2006. Global optimization for the synthesis of integrated water systems in chemical processes. Comput. Chem. Eng. 30, 650 $-673$.

Kelley, C., 2011. Implicit Filtering. Society for Industrial and Applied Mathematics.

Khajavirad, A., Sahinidis, N. V., 2012. Convex envelopes of products of convex and component-wise concave functions. J. Glob. Optim. 52, 391-409.

Khajavirad, A., Sahinidis, N. V., 2013. Convex envelopes generated from finitely many compact convex sets. Math. Program. 137 (1-2), 371-408.

Khor, C. S., Chachuat, B., Shah, N., 2014. Fixed-flowrate total water network synthesis under uncertainty with risk management. J. Clean. Prod. 77 (0), 79 93.

Kleijnen, J. P. C., van Beers, W., van Nieuwenhuyse, I., 2010. Constrained optimization in expensive simulation: Novel approach. Eur. J. Oper. Res. 202 (1), 164-174.

Kleniati, P., Parpas, P., Rustem, B., 2010. Decomposition-based method for sparse semidefinite relaxations of polynomial optimization problems. J. Optim. Theory Appl. 145 (2), 289-310.

Kleniati, P.-M., Adjiman, C. S., 2014a. Branch-and-sandwich: a deterministic global optimization algorithm for optimistic bilevel programming problems. Part I: Theoretical development. J. Glob. Optim. 60 (3), 425-458. 
Kleniati, P.-M., Adjiman, C. S., 2014b. Branch-and-sandwich: a deterministic global optimization algorithm for optimistic bilevel programming problems. Part II: Convergence analysis and numerical results. J. Glob. Optim. 60 (3), 425-458.

Klepeis, J. L., Floudas, C. A., Morikis, D., Tsokos, C. G., Lambris, J. D., 2004. Design of peptide analogues with improved activity using a novel de novo protein design approach. Ind. Eng. Chem. Res. 43 (14), 3817-3826.

Klepeis, J. L., Pieja, M. J., Floudas, C. A., 2003a. Hybrid global optimization algorithms for protein structure prediction: Alternating hybrids. Biophysical Journal 84 (2), $869-882$.

Klepeis, J. L., Pieja, M. J., Floudas, C. A., 2003b. A new class of hybrid global optimization algorithms for peptide structure prediction: integrated hybrids. Computer Physics Communications 151 (2), 121 - 140.

Kocis, G. R., Grossmann, I. E., 1988. Global optimization of nonconvex mixedinteger nonlinear programming (MINLP) problems in process synthesis. Ind. Eng. Chem. Res. 27 (8), 1407-1421.

Kokkolaras, M., Audet, C., Dennis, J.E., J., 2001. Mixed variable optimization of the number and composition of heat intercepts in a thermal insulation system. Optimization and Engineering 2 (1), 5-29.

Kokossis, A. C., Floudas, C. A., 1991. Synthesis of isothermal reactor-separatorrecycle systems. Chem. Eng. Sci. 46 (5 - 6), 1361 - 1383.

Kokossis, A. C., Floudas, C. A., 1994. Optimization of complex reactor networksII. Nonisothermal operation. Chem. Eng. Sci. 49 (7), 1037 - 1051.

Kolda, T. G., Lewis, R. M., Torczon, V., 2003. Optimization by direct search: New perspectives on some classical and modern methods. SIAM Review 45 (3), 385-482.

Kolda, T. G., Lewis, R. M., Torczon, V., 2006. Stationarity results for generating set search for linearly constrained optimization. SIAM J. Optim. 17 (4), 943968.

Kolodziej, S. P., Castro, P. M., Grossmann, I. E., 2013a. Global optimization of bilinear programs with a multiparametric disaggregation technique. J. Glob. Optim. 57 (4), 1039-1063.

Kolodziej, S. P., Grossmann, I. E., Furman, K. C., Sawaya, N. W., 2013b. A discretization-based approach for the optimization of the multiperiod blend scheduling problem. Comput. Chem. Eng. 53, $122-142$.

Koziel, V., Yang, X. S. (Eds.), 2011. Computational Optimization, Methods and 
Algorithms. Vol. 356 of Studies in Computational Intelligence. Springer.

Laguna, M., Gortźar, F., Gallego, M., Duarte, A., Martí, R., 2014. A black-box scatter search for optimization problems with integer variables. J. Glob. Optim. 58 (3), 497-516.

Lasserre, J., 2006. Convergent SDP relaxations in polynomial optimization with sparsity. SIAM J. Optim. 17 (3), 822-843.

Le Digabel, S., 2011. Algorithm 909: NOMAD: Nonlinear optimization with the MADS algorithm. ACM Transactions on Mathematical Software 37 (4), 1-15.

Le Thi, H. A., Vaz, A. I. F., Vicente, L. N., 2012. Optimizing radial basis functions by D.C. programming and its use in direct search for global derivative-free optimization. TOP 20 (1), 190-214.

Lebbah, Y., Michel, C., Rueher, M., 2005. A rigorous global filtering algorithm for quadratic constraints. Constraints 10 (1), 47-65.

Lee, H., Pinto, J. M., Grossmann, I. E., Park, S., 1996. Mixed-integer linear programming model for refinery short-term scheduling of crude oil unloading with inventory management. Ind. Eng. Chem. Res. 35 (5), 1630-1641.

Lee, J., Leyffer, S. (Eds.), 2012. Mixed Integer Nonlinear Programming. The IMA Volumes in Mathematics and its Applications. Springer.

Lewis, R., Shepherd, A., Torczon, V., 2007. Implementing generating set search methods for linearly constrained minimization. SIAM J. Sci. Comput. 29 (6), 2507-2530.

Lewis, R., Torczon, V., 1999. Pattern search algorithms for bound constrained minimization. SIAM J. Optim. 9 (4), 1082-1099.

Lewis, R., Torczon, V., 2000. Pattern search methods for linearly constrained minimization. SIAM J. Optim. 10 (3), 917-941.

Leyffer, S., Linderoth, J., Luedtke, J., Miller, A., Munson, T., 2009. Applications and algorithms for mixed integer nonlinear programming. Journal of Physics: Conference Series 180 (1), 012014.

Li, J., Li, A., Karimi, I. A., Srinivasan, R., 2007. Improving the robustness and efficiency of crude scheduling algorithms. AIChE J. 53 (10), 2659-2680.

Li, J., Misener, R., Floudas, C. A., 2012a. Continuous-time modeling and global optimization approach for scheduling of crude oil operations. AIChE J. 58 (1), 205-226.

Li, J., Misener, R., Floudas, C. A., 2012b. Scheduling of crude oil operations under demand uncertainty: A robust optimization framework coupled with global optimization. AIChE J. 58 (8), 2373-2396. 
Li, X., Armagan, E., Tomasgard, A., Barton, P. I., 2011. Stochastic pooling problem for natural gas production network design and operation under uncertainty. AIChE J. 57 (8), 2120-2135.

Li, X., Tomasgard, A., Barton, P. I., 2012c. Decomposition strategy for the stochastic pooling problem. J. Glob. Optim. 54 (4), 765-790.

Liberti, L., 2012. Reformulations in mathematical programming: automatic symmetry detection and exploitation. Math. Program. 131 (1-2), 273-304.

Liberti, L., Cafieri, S., Savourey, D., 2010. The reformulation-optimization software engine. In: Proceedings of the Third international congress conference on Mathematical software. ICMS'10. pp. 303-314.

Liberti, L., Cafieri, S., Tarissan, F., 2009a. Reformulations in mathematical programming: A computational approach. In: Abraham, A., Hassanien, A.-E., Siarry, P., Engelbrecht, A. (Eds.), Foundations of Computational Intelligence Volume 3. Vol. 203 of Studies in Computational Intelligence. Springer, Berlin, pp. 153-234.

Liberti, L., Lavor, C., Maculan, N., Nascimento, M., 2009b. Reformulation in mathematical programming: an application to quantum chemistry. Discrete Applied Mathematics 157 (6), 1309-1318.

Liberti, L., Maculan, N., 2006. Global Optimization: From Theory to Implementation. Nonconvex Optimization and Its Applications. Springer.

Liberti, L., Mladenović, N., Nannicini, G., 2011. A recipe for finding good solutions to MINLPs. Math. Program. Comput. 3, 349-390.

Liberti, L., Pantelides, C. C., 2003. Convex envelopes of monomials of odd degree. J. Glob. Optim. 25, 157-168.

Liberti, L., Pantelides, C. C., 2006. An exact reformulation algorithm for large nonconvex NLPs involving bilinear terms. J. Glob. Optim. 36 (2), 161-189.

Lima, R. M., Francois, G., Srinivasan, B., Salcedo, R. L., 2004. Dynamic optimization of batch emulsion polymerization using MSIMPSA, a simulatedannealing-based algorithm. Ind. Eng. Chem. Res. 43 (24), 7796-7806.

Lin, X., Floudas, C. A., 2001. Design, synthesis and scheduling of multipurpose batch plants via an effective continuous-time formulation. Comput. Chem. Eng. 25 (4 - 6), $665-674$.

Lin, X., Floudas, C. A., Kallrath, J., 2005. Global solution approach for a nonconvex MINLP problem in product portfolio optimization. J. Glob. Optim. 32, 417-431.

Lin, Y., Schrage, L., 2009. The global solver in the LINDO API. Optim. Method. 
Linderoth, J., 2005. A simplicial branch-and-bound algorithm for solving quadratically constrained quadratic programs. Math. Program. 103 (2), 251 - 282.

Liu, W. B., Floudas, C. A., 1993. A remark on the GOP algorithm for global optimization. J. Glob. Optim. 3, 519-521.

Liuzzi, G., Lucidi, S., 2009. A derivative-free algorithm for inequality constrained nonlinear programming via smoothing of an $1-\infty$ penalty function. SIAM Journal on Optimization 20 (1), 1-29.

Liuzzi, G., Lucidi, S., Piccialli, V., 2015a. Exploiting derivative-free local searches in direct-type algorithms for global optimization. Computational Optimization and Applications, 1-27.

URL http: / / dx.doi.org/10.1007/s10589-015-9741-9

Liuzzi, G., Lucidi, S., Rinaldi, F., 2012. Derivative-free methods for bound constrained mixed-integer optimization. Comput. Optim. Appl. 53 (2), 505-526.

Liuzzi, G., Lucidi, S., Rinaldi, F., 2015b. Derivative-free methods for mixedinteger constrained optimization problems. J Optim Theory Appl 164 (3), 933965.

Liuzzi, G., Lucidi, S., Sciandrone, M., 2010. Sequential penalty derivative-free methods for nonlinear constrained optimization. SIAM J. Optim. 20 (5), 26142635.

Locatelli, M., 2013. Computing the value of the convex envelope of quadratic forms over polytopes through a semidefinite program. Oper. Res. Lett. 41 (4), $370-372$.

Locatelli, M., 2014. A technique to derive the analytical form of convex envelopes for some bivariate functions. J. Glob. Optim. 59 (2-3), 477-501.

Locatelli, M., 2015. Alternative branching rules for some nonconvex problems. Optim. Method. Softw. 30 (2), 365-378.

Locatelli, M., Schoen, F., 2013. Global Optimization: Theory, Algorithms, and Applications. Vol. 15. SIAM.

Locatelli, M., Schoen, F., 2014. On convex envelopes for bivariate functions over polytopes. Math. Program. 144 (1-2), 65-91.

Loiola, E. M., de Abreu, N. M. M., Boaventura-Netto, P. O., Hahn, P., Querido, T., 2007. A survey for the quadratic assignment problem. Eur. J. Oper. Res. 176 (2), $657-690$.

Lovász, L., 1982. Submodular functions and convexity. In: Grötschel, M., Kor, B. (Eds.), Mathematical Programming: The State of the Art. Springer, pp. 235- 
257.

Lucidi, S., Piccialli, V., Sciandrone, M., 2005. An algorithm model for mixed variable programming. SIAM J. Optim. 15 (4), 1057-1084.

Lucidi, S., Sciandrone, M., 2002. A derivative-free algorithm for bound constrained optimization. Comput. Optim. Appl. 21 (2), 119-142.

Luedtke, J., Namazifar, M., Linderoth, J., 2012. Some results on the strength of relaxations of multilinear functions. Math. Program. 136 (2), 325-351.

Lundell, A., Skjäl, A., Westerlund, T., 2013. A reformulation framework for global optimization. J. Glob. Optim. 57 (1), 115-141.

Lundell, A., Westerlund, J., Westerlund, T., 2009. Some transformation techniques with applications in global optimization. J. Glob. Optim. 43, 391-405.

Lundell, A., Westerlund, T., 2009. Convex underestimation strategies for signomial functions. Optim. Method. Softw. 24 (4-5), 505-522.

Lundell, A., Westerlund, T., 2012. Global optimization of mixed-integer signomial programming problems. In: Lee, J., Leyffer, S. (Eds.), Mixed Integer Nonlinear Programming. Vol. 154 of The IMA Volumes in Mathematics and its Applications. Springer New York, pp. 349-369.

Maleki, S., Sawhney, R., Farvaresh, H., Sepehri, M. M., 2014. Energy efficient hybrid wired-cum-wireless sensor network design. J. Clean. Prod. 85 (0), 408 $-418$.

Maranas, C. D., Androulakis, I. P., Floudas, C. A., Berger, A. J., Mulvey, J. M., 1997. Solving long-term financial planning problems via global optimization. Journal of Economic Dynamics and Control 21 (8-9), 1405 - 1425.

Maranas, C. D., Floudas, C. A., 1992. A global optimization approach for Lennard-Jones microclusters. J. Chem. Phys. 97 (10), 7667-7678.

Maranas, C. D., Floudas, C. A., 1995. Finding all solutions of nonlinearly constrained systems of equations. J. Glob. Optim. 7 (2), 143-182.

Maranas, C. D., Floudas, C. A., 1997. Global optimization in generalized geometric programming. Comput. Chem. Eng. 21 (4), $351-369$.

March, A., Willcox, K., 2012. Constrained multifidelity optimization using model calibration. Struct. Multidiscip. Optim. 46 (1), 93-109.

Marsden, A. L., Feinstein, J. A., Taylor, C. A., 2008. A computational framework for derivative-free optimization of cardiovascular geometries. Computer Methods in Applied Mechanics and Engineering 197 (21-24), 1890-1905.

Marsden, A. L., Wang, M., Dennis, J. E., Parviz, M., 2004. Optimal aerocoustic 
shape design using surrogate management framework. Optim. Eng. 5 (2), 235262.

Marsden, A. L., Wang, M., Dennis, J. E., Parviz, M., 2007. Trailing-edge noise reduction using derivative-free optimization and large-eddy simulation. J. Fluid Mech. 572, 13-36.

Martelli, E., Amaldi, E., 2014. PGS-COM: A hybrid method for constrained nonsmooth black-box optimization problems: Brief review, novel algorithm and comparative evaluation. Comput. Chem. Eng. 63, 108-139.

Martelli, E., Amaldi, E., Consonni, S., 2011. Numerical optimization of heat recovery steam cycles: Mathematical model, two-stage algorithm and applications. Comput. Chem. Eng. 35 (12), 2799 - 2823.

Martinez, J. M., Sobral, F. N. C., 2013. Constrained derivative-free optimization on thin domains. J. Glob. Optim. 56 (3), 1217-1232.

McCormick, G. P., 1976. Computability of global solutions to factorable nonconvex programs: Part 1-convex underestimating problems. Math. Program. 10 (1), $147-175$.

McDonald, C. M., Floudas, C. A., 1994. Decomposition based and branch and bound global optimization approaches for the phase equilibrium problem. J. Glob. Optim. 5 (3), 205-251.

McDonald, C. M., Floudas, C. A., 1995a. Global optimization for the phase and chemical equilibrium problem: Application to the NRTL equation. Comput. Chem. Eng. 19 (11), $1111-1139$.

McDonald, C. M., Floudas, C. A., 1995b. Global optimization for the phase stability problem. AIChE J. 41 (7), 1798-1814.

McDonald, C. M., Floudas, C. A., 1996. GLOPEQ: A new computational tool for the phase and chemical equilibrium problem. Comput. Chem. Eng. 21 (1), 1 23.

Meyer, C. A., Floudas, C. A., 2003. Trilinear monomials with positive or negative domains: Facets of the convex and concave envelopes. In: Floudas, C. A., Pardalos, P. M. (Eds.), Frontiers in Global Optimization. Kluwer Academic Publishers, pp. 327-352.

Meyer, C. A., Floudas, C. A., 2004. Trilinear monomials with mixed sign domains: Facets of the convex and concave envelopes. J. Glob. Optim. 29 (2), 125-155.

Meyer, C. A., Floudas, C. A., 2005a. Convex envelopes for edge-concave functions. Math. Program. 103 (2), 207-224. 
Meyer, C. A., Floudas, C. A., 2005b. Convex underestimation of twice continuously differentiable functions by piecewise quadratic perturbation: Spline $\alpha \mathrm{BB}$ underestimators. J. Glob. Optim. 32 (2), 221-258.

Meyer, C. A., Floudas, C. A., 2006. Global optimization of a combinatorially complex generalized pooling problem. AIChE J. 52 (3), 1027 - 1037.

Meza, J. C., Martinez, M. L., 1994. Direct search methods for the molecular conformation problem. Journal of Computational Chemistry 15 (6), 627-632.

Misener, R., Floudas, C. A., 2009. Advances for the pooling problem: Modeling, global optimization, and computational studies. Applied and Computational Mathematics 8 (1), 3 - 22.

Misener, R., Floudas, C. A., 2010. Global optimization of large-scale pooling problems: Quadratically constrained MINLP models. Ind. Eng. Chem. Res. 49 (11), $5424-5438$.

Misener, R., Floudas, C. A., 2012a. Global optimization of mixed-integer models with quadratic and signomial functions: A review. Applied and Computational Mathematics 11 (3), 317-336.

Misener, R., Floudas, C. A., 2012b. Global optimization of mixed-integer quadratically-constrained quadratic programs (MIQCQP) through piecewiselinear and edge-concave relaxations. Math. Program. B 136, 155-182.

Misener, R., Floudas, C. A., 2013. GloMIQO: Global Mixed-Integer Quadratic Optimizer. J. Glob. Optim. 57 (1), 3-50.

Misener, R., Floudas, C. A., 2014a. ANTIGONE: Algorithms for coNTinuous Integer Global Optimization of Nonlinear Equations. J. Glob. Optim. 59 (2-3), 503-526.

Misener, R., Floudas, C. A., 2014b. A framework for globally optimizing mixedinteger signomial programs. J. Optim. Theory Appl. 161, 905-932.

Misener, R., Garí, M. F., Rende, M., Velliou, E., Panoskaltsis, N., Pistikopoulos, E. N., Mantalaris, A., 2014a. Global superstructure optimisation of red blood cell production in a parallelised hollow fibre bioreactor. Comput Chem Eng 71, $532-553$.

Misener, R., Gounaris, C. E., Floudas, C. A., 2010. Mathematical modeling and global optimization of large-scale extended pooling problems with the (EPA) complex emissions constraints. Comput. Chem. Eng. 34 (9), 1432 - 1456.

Misener, R., Smadbeck, J. B., Floudas, C. A., 2014b. Dynamically-generated cutting planes for mixed-integer quadratically-constrained quadratic programs and their incorporation into GloMIQO 2.0. Optim. Method. Softw. 30 (1), 215-249. 
Misener, R., Thompson, J. P., Floudas, C. A., 2011. APOGEE: Global optimization of standard, generalized, and extended pooling problems via linear and logarithmic partitioning schemes. Comput. Chem. Eng. 35 (5), 876-892.

Mitsos, A., Barton, P. I., 2007. A dual extremum principle in thermodynamics. AIChE J. 53 (8), 2131-2147.

Mitsos, A., Chachuat, B., Barton, P. I., 2009. McCormick-based relaxations of algorithms. SIAM J. Optim. 20 (2), 573-601.

Moles, C. G., Mendes, P., Banga, J. R., 2003. Parameter estimation in biochemical pathways: A comparison of global optimization methods. Genome Research 13, 2467-2474.

Moré, J. J., Wild, S. M., 2002. Benchmarking derivative-free optimization algorithms. SIAM Journal on Optimization 91, 201-213.

Morin, A., Wahl, P. E., Malnvik, M., 2011. Using evolutionary search to optimise the energy consumption for natural gas liquefaction. Chem. Eng. Res. Des. 89 (1), $2428-2441$.

Mouret, S., Grossmann, I. E., 2010. Crude-oil operations scheduling. Available from CyberInfrastructure for MINLP [A collaboration of Carnegie Mellon University and IBM Research] at: www.minlp.org/library/problem/index.php? $i=117$.

Mouret, S., Grossmann, I. E., Pestiaux, P., 2009. A novel priority-slot based continuous-time formulation for crude-oil scheduling problems. Ind. Eng. Chem. Res. 48 (18), 8515-8528.

Mouret, S., Grossmann, I. E., Pestiaux, P., 2011. A new Lagrangian decomposition approach applied to the integration of refinery planning and crude-oil scheduling. Comput. Chem. Eng. 35 (12), 2750-2766.

Mugunthan, P., Shoemaker, C. A., Regis, R. G., 2005. Comparison of function approximation, heuristic, and derivative-based methods for automatic calibration of computationally expensive groundwater bioremediation models. Water Resources Research 41 (11), W11427.

Müller, J., Shoemaker, C. A., 2014. Influence ensemble surrogate models and sampling strategy on the solution quality of algorithms for computationally expensive black-box global optimization methods. J. Glob. Optim. 60 (2), 123 144.

Müller, J., Shoemaker, C. A., Piche, R., 2013. SO-MI: A surrogate model algorithm for computationally expensive nonlinear mixed-integer black-box global optimization problems. Comput. Oper. Res. 40 (5), 1383-1400. 
Nannicini, G., Belotti, P., 2012. Rounding-based heuristics for nonconvex MINLPs. Math. Program. Comput. 4 (1), 1-31.

Nelder, J. A., Mead, R., 1965. A simplex method for function minimization. Comput. J. 7, 308-313.

Neumaier, A., 1990. Interval Methods for Systems of Equations. Encyclopedia of Mathematics and its Applications. Cambridge University Press, Cambridge.

Newby, E., Ali, M. M., 2014. A trust-region-based derivative free algorithm for mixed integer programming. Comput. Optim. Appl. 60 (1), 199-229.

Nicosia, G., Stracquadanio, G., 2008. Generalized pattern search algorithm for peptide structure prediction. Biophysical Journal 95 (10), 4988 - 4999.

Niknam, T., Khodaei, A., Fallahi, F., 2009. A new decomposition approach for the thermal unit commitment problem. Applied Energy 86 (9), 1667 - 1674.

Niziolek, A. M., Onel, O., Elia, J. A., Baliban, R. C., Xiao, X., Floudas, C. A., 2014. Coal and biomass to liquid transportation fuels: Process synthesis and global optimization strategies. Ind. Eng. Chem. Res. 53 (44), 17002-17025.

Nowak, I., 2005. Relaxation and decomposition methods for mixed integer nonlinear programming. International series of numerical mathematics. Birkhäuser.

Nyberg, A., Grossmann, I. E., Westerlund, T., 2012. The optimal design of a three-echelon supply chain with inventories under uncertainty. Available from CyberInfrastructure for MINLP [www.minlp.org, a collaboration of Carnegie Mellon University and IBM Research] at: www.minlp.org/library/problem/index.php? $i=157$.

Nyberg, A., Grossmann, I. E., Westerlund, T., 2013. An efficient reformulation of the multiechelon stochastic inventory system with uncertain demands. AIChE J. 59 (1), 23-28.

Oeuvray, R., 2005. Trust-region methods based on radial basis functions with application to biomedical imaging. PhD in Mathematics, Ecole Polytechnique Federale de Lausanne.

Oeuvray, R., Bierlaire, M., 2007. A new derivative-free algorithm for the medical image registration problem. International Journal of Modeling and Simulation 27 (2).

Onel, O., Niziolek, A. M., Hasan, M. M. F., Floudas, C. A., 2014. Municipal solid waste to liquid transportation fuels part I: Mathematical modeling of a municipal solid waste gasifier. Comput. Chem. Eng. 71, 636 - 647.

Ong, Y. S., Nair, P. B., Keane, A. J., 2003. Evolutionary optimization of computationally expensive problems via surrogate modeling. AIAA J. 41 (4), 687-696. 
Pal, L., Csendes, T., Markot, M. C., Neumaier, A., 2012. Black box optimization benchmarking of the GLOBAL method. Evol. Comput. 20 (4), 609-639.

Papadopoulos, A. I., Linke, P., 2004. On the synthesis and optimization of liquidliquid extraction processes using stochastic search methods. Comput. Chem. Eng. 28 (11), $2391-2406$.

Papageorgiou, D. J., Toriello, A., Nemhauser, G. L., Savelsbergh, M. W. P., 2012. Fixed-charge transportation with product blending. Transport. Sci. 46 (2), 281295.

Pardalos, P. M., 1991. Global optimization algorithms for linearly constrained indefinite quadratic problems. Comput. Math. Appl. 21 (6-7), 87 - 97.

Parpas, P., Rustem, B., 2006. Global optimization of the scenario generation and portfolio selection problems. In: Gavrilova, M., Gervasi, O., Kumar, V., Tan, C., Taniar, D., Laganá, A., Mun, Y., Choo, H. (Eds.), Computational Science and Its Applications - ICCSA 2006. Vol. 3982 of Lecture Notes in Computer Science. Springer-Verlag, pp. 908-917.

Parr, J. M., Keane, A. J., Forrester, A. I. J., Holden, C. M. E., 2012. Infill sampling criteria for surrogate-based optimization with constraint handling. Eng. Optim. 44 (10), 1147-1166.

Parrilo, P. A., 2003. Semidefinite programming relaxations for semialgebraic problems. Math. Program. 96, 293-320.

Pereira, F. E., Jackson, G., Galindo, A., Adjiman, C. S., 2010. A duality-based optimisation approach for the reliable solution of $(\mathrm{p}, \mathrm{t})$ phase equilibrium in volume-composition space. Fluid Phase Equilibr. 299 (1), 1 - 23.

Pfetsch, M. E., Fügenschuh, A., Geissler, B., Geissler, N., Gollmer, R., Hiller, B., Humpola, J., Koch, T., Lehmann, T., Martin, A., Morsi, A., Rövekamp, J., Schewe, L., Schmidt, M., Schultz, R., Schwarz, R., Schweiger, J., Stangl, C., Steinbach, M. C., Vigerske, S., Willert, B. M., 2015. Validation of nominations in gas network optimization: models, methods, and solutions. Optim Met Softw 30 (1), 15-53.

Pham, V., Laird, C., El-Halwagi, M., 2009. Convex hull discretization approach to the global optimization of pooling problems. Ind. Eng. Chem. Res. 48, 1973 $-1979$.

Plantenga, T. D., 2009. HOPSPACK 2.0 User Manual. Available at: http: // www.sandia.gov/hopspack/HopspackUserManual_2_0_2.pdf.

Polisetty, P. K., Gatzke, E. P., Voit, E. O., 2008. Yield optimization of regulated metabolic systems using deterministic branch-and-reduce methods. Biotechnol. 
Bioeng. 99 (5), 1154-1169.

Ponce-Ortega, J. M., El-Halwagi, M. M., Jiménez-Gutiérrez, A., 2010. Global optimization for the synthesis of property-based recycle and reuse networks including environmental constraints. Comput. Chem. Eng. 34 (3), 318 - 330.

Powell, M. J. D., 1994. A direct search optimization method that models the objective and constraint functions by linear interpolation. In: Advances in Optimization and Numerical Analysis. Vol. 275 of Mathematics and Its Applications. Springer Netherlands, pp. 51-67.

Powell, M. J. D., 2009. The BOBYQA algorithm for bound constrained optimization without derivativesAvailable at: www. damtp.cam.ac.uk/user/na/ NA_papers/NA2009_06.pdf.

Powell, M. J. D., 2013a. COBYLA (constrained optimization by linear approximations). Available at: http://mat.uc.pt/ zhang/software. html\#cobyla.

Powell, M. J. D., 2013b. LINCOA (linearly constrained optimization algorithm). Available at: http://mat.uc.pt/ zhang/software.html\# lincoa.

Pozo, C., Guillen-Gosalbez, G., Sorribas, A., Jimenez, L., 2011. A spatial branch-and-bound framework for the global optimization of kinetic models of metabolic networks. Ind. Eng. Chem. Res. 50 (9), 5225-5238.

Qualizza, A., Belotti, P., Margot, F., 2012. Linear programming relaxations of quadratically constrained quadratic programs. In: Lee, J., Leyffer, S. (Eds.), Mixed Integer Nonlinear Programming. Vol. 154 of The IMA Volumes in Mathematics and its Applications. Springer New York, pp. 407-426.

Quan, N., Yin, J., Ng, S. H., Lee, L. H., 2013. Simulation optimization via kriging: a sequential search using expected improvement with computing budget constraints. IIE Transactions 45 (7), 763-780.

Quesada, I., Grossmann, I. E., 1995. A global optimization algorithm for linear fractional and bilinear programs. J. Glob. Optim. 6 (1), 39-76.

Rashid, K., Ambani, S., Cetinkaya, E., 2013. An adaptive multiquadric radial basis function method for expensive black-box mixed-integer nonlinear constrained optimization. Eng. Optim. 45 (2), 185-206.

Rebennack, S., Kallrath, J., Pardalos, P. M., 2009. Column enumeration based decomposition techniques for a class of non-convex MINLP problems. J. Glob. Optim. 43 (2-3), 277-297.

Regis, R. G., 2011. Stochastic radial basis function algorithms for large-scale op- 
timization involving expensive black-box objective and constraint functions. Comput. Oper. Res. 38 (5), 837-853.

Regis, R. G., 2014. Constrained optimization by radial basis function interpolation for high-dimensional expensive black-box problems with infeasible initial points. Eng. Optim. 46 (2), 218-243.

Regis, R. G., Shoemaker, C. A., 2005. Constrained global optimization of expensive black box functions using radial basis functions. J. Glob. Optim. 31 (1), 153-171.

Regis, R. G., Shoemaker, C. A., 2007a. Improved strategies for radial basis function methods for global optimization. J. Glob. Optim. 37 (1), 113-135.

Regis, R. G., Shoemaker, C. A., 2007b. Parallel radial basis function methods for the global optimization of expensive functions. Eur. J. Oper. Res. 182 (2), 514-535.

Regis, R. G., Shoemaker, C. A., 2007c. A stochastic radial basis function method for the global optimization of expensive functions. INFORMS J. Comput. 19 (4), 497-509.

Regis, R. G., Shoemaker, C. A., 2013a. Combining radial basis function surrogates and dynamic coordinate search in high-dimensional expensive black-box optimization. Engineering Optimization 45 (5), 529-555.

Regis, R. G., Shoemaker, C. A., 2013b. A quasi-multistart framework for global optimization of expensive functions using response surface models. J. Glob. Optim. 56 (4), 1719-1753.

Rendl, F., Rinaldi, G., Wiegele, A., 2010. Solving max-cut to optimality by intersecting semidefinite and polyhedral relaxations. Math. Program. 121 (2), 307335.

Richard, J. P., Tawarmalani, M., 2010. Lifting inequalities: a framework for generating strong cuts for nonlinear programs. Math. Program. 121 (1), 61-104.

Rikun, A. D., 1997. A convex envelope formula for multilinear functions. J. Glob. Optim. 10, $425-437$.

Rios, L. M., Sahinidis, N. V., 2010. Portfolio optimization for wealth-dependent risk preferences. Annals of Oper. Res. 177, 63-90.

Rios, L. M., Sahinidis, N. V., 2013. Derivative-free optimization: a review of algorithms and comparison of software implementations. J. Glob. Optim. 56 (3), 1247-1293.

Rodrigues-Fernandez, M. P., Banga, J. R., 2006. A hybrid approach for efficient and robust parameter estimation in biochemical pathways. Biosystems 83, 248- 
265.

Rodrigues-Fernandez, M. P., Egea, J., Banga, J. R., 2006. Novel metaheuristic for parameter estimation in nonlinear dynamic biological systems. BMC Bioinformatics 7 (1), 483.

Romero, R., Monticelli, A., Garcia, A., Haffner, S., 2002. Test systems and mathematical models for transmission network expansion planning. Generation, Transmission and Distribution, IEE Proceedings- 149 (1), 27-36.

Rosen, J. B., Pardalos, P. M., 1986. Global minimization of large-scale constrained concave quadratic problems by separable programming. Math. Program. 34 (2), 163-174.

Ruiz, J. P., Grossmann, I. E., 2009. Water treatment network design. Available from CyberInfrastructure for MINLP [wWw.minlp.org, a collaboration of Carnegie Mellon University and IBM Research] at: www.minlp.org/library/problem/index.php?i=24.

Ruiz, J. P., Grossmann, I. E., 2011a. Exploiting vector space properties to strengthen the relaxation of bilinear programs arising in the global optimization of process networks. Optim. Lett. 5, 1-11.

Ruiz, J. P., Grossmann, I. E., 2011b. Using redundancy to strengthen the relaxation for the global optimization of MINLP problems. Comput. Chem. Eng. 35, 2729-2740.

Ruiz, M., Briant, O., Clochard, J.-M., Penz, B., 2013. Large-scale standard pooling problems with constrained pools and fixed demands. J. Glob. Optim. 56 (3), 939-956.

Ruuth, S., 2006. Global optimization of explicit strong-stability-preserving Runge-Kutta methods. Math. Comput. 75 (253), 183-207.

Ryoo, H. S., Sahinidis, N. V., 1995. Global optimization of nonconvex NLPs and MINLPs with applications in process design. Comput. Chem. Eng. 19 (5), 551566.

Ryoo, H. S., Sahinidis, N. V., 1996. A branch-and-reduce approach to global optimization. J. Glob. Optim. 8, 107-138.

Ryoo, H. S., Sahinidis, N. V., 2001. Analysis of bounds for multilinear functions. J. Glob. Optim. 19 (4), 403-424.

Sahinidis, N. V., 1996. BARON: A general purpose global optimization software package. J. Glob. Optim. 8 (2), 201-205.

Saif, Y., Elkamel, A., Pritzker, M., 2008. Global optimization of reverse osmosis network for wastewater treatment and minimization. Ind. Eng. Chem. Res. 
47 (9), 3060 - 3070.

Sankaran, S., Audet, C., Marsden, A. L., 2010. A method for stochastic constrained optimization using derivative-free surrogate pattern search and collocation. J. Comput. Phys. 229 (12), 4664-4682.

Sasena, M. J., Papalambros, P., Goovaerts, P., 2002. Exploration of metamodeling sampling criteria for constrained global optimization. Eng. Optim. 34 (3), 263278.

Savola, T., Fogelholm, C.-J., 2007. MINLP optimisation model for increased power production in small-scale CHP plants. Appl. Therm. Eng. 27 (1), 89 99.

Sawaya, N. W., 2006. Reformulations, relaxations and cutting planes for generalized disjunctive programming. $\mathrm{PhD}$ in Chemical Engineering, Carnegie Mellon University.

Saxena, A., Bonami, P., Lee, J., 2010. Convex relaxations of non-convex mixed integer quadratically constrained programs: Extended formulations. Math. Program. 124 (1-2), 383-411.

Saxena, A., Bonami, P., Lee, J., 2011. Convex relaxations of non-convex mixed integer quadratically constrained programs: Projected formulations. Math. Program. 130, 359-413.

Schittkowski, K., 1987. More Test examples for nonlinear programming codes. Vol. 282 of Lecture Notes in Economics and Mathematical Systems. SpringerVerlag.

Scott, J., Stuber, M., Barton, P. I., 2011. Generalized McCormick relaxations. J. Glob. Optim. 51, 569-606.

Scozzari, A., Tardella, F., 2008. A clique algorithm for standard quadratic programming. Discrete Applied Mathematics 156 (13), 2439 - 2448.

Selot, A., Kuok, L. K., Robinson, M., Mason, T. L., Barton, P. I., 2008. A shortterm operational planning model for natural gas production systems. AIChE J. 54 (2), 495-515.

Shah, S. B., Sahinidis, N. V., 2012. SAS-Pro: Simultaneous residue assignment and structure superposition for protein structure alignment. PLoS ONE 7 (5), e37493, doi:10.1371/journal.pone.0037493.

Sherali, H., Dalkiran, E., Liberti, L., 2012. Reduced RLT representations for nonconvex polynomial programming problems. J. Glob. Optim. 52 (3), 447-469.

Sherali, H. D., 1997. Convex envelopes of multilinear functions over a unit hypercube and over special discrete sets. Acta Mathematica Vietnamica 22 (1), 
245-270.

Sherali, H. D., Adams, W. P., 1999. A Reformulation-Linearization Technique for Solving Discrete and Continuous Nonconvex Problems. Nonconvex Optimization and Its Applications. Kluwer Academic Publishers, Dordrecht, Netherlands.

Sherali, H. D., Alameddine, A., 1992. A new reformulation-linearization technique for bilinear programming problems. J. Glob. Optim. 2, 379 - 410.

Sherali, H. D., Fraticelli, B. M. P., 2002. Enhancing RLT relaxations via a new class of semidefinite cuts. J. Glob. Optim. 22 (1-4), 233-261.

Sherali, H. D., Tuncbilek, C. H., 1995. A reformulation-convexification approach for solving nonconvex quadratic-programming problems. J. Glob. Optim. 7 (1), $1-31$.

Sherali, H. D., Tuncbilek, C. H., 1997. New reformulation linearization/convexification relaxations for univariate and multivariate polynomial programming problems. Oper. Res. Lett. 21 (1), 1 - 9.

Shikhman, V., Stein, O., 2012. On jet-convex functions and their tensor products. Optim. 61 (6), 717-731.

Skjäl, A., Westerlund, T., 2014. New methods for calculating $\alpha$ BB-type underestimators. J. Glob. Optim. 58 (3), 411-427.

Skjäl, A., Westerlund, T., Misener, R., Floudas, C. A., 2012. A generalization of the classical $\alpha \mathrm{BB}$ convex underestimation via diagonal and nondiagonal quadratic terms. J. Optim. Theory Appl. 154 (2), 462-490.

Smith, E. M. B., Pantelides, C. C., 1997. Global optimisation of nonconvex MINLPs. Comput. Chem. Eng. 21, Supplement, S791 - S796.

Smith, E. M. B., Pantelides, C. C., 1999. A symbolic reformulation/spatial branchand-bound algorithm for the global optimisation of nonconvex MINLPs. Comput. Chem. Eng. 23 (4 - 5), 457 - 478.

Spendley, W., Hext, G. R., Himsworth, F. R., 1962. Sequential application of simplex designs in optimisation and evolutionary operation. Technometrics 4 (4), 441-461.

Sun, J., Garibaldi, J. M., Krasnogor, N., Zhang, Q., 2013. An intelligent multirestart memetic algorithm for box constrained global optimisation. Evolutionary Computation 21 (1), 107-147.

Szabó, P. G., Markót, C. M., Csendes, T., 2005. Global optimization in geometry circle packing into the square. In: Audet, C., Hansen, P., Savard, G. (Eds.), Essays and Surveys in Global Optimization. Springer, New York, pp. 233-265. 
Tadayon, B., Smith, J. C., 2013. Algorithms for an integer multicommodity network flow problem with node reliability considerations. J. Optim. Theory Appl. $161,506-532$.

Tardella, F., 1988/89. On a class of functions attaining their maximum at the vertices of a polyhedron. Discret. Appl. Math. 22, 191-195.

Tardella, F., 2003. On the existence of polyhedral convex envelopes. In: Floudas, C. A., Pardalos, P. M. (Eds.), Frontiers in Global Optimization. Kluwer Academic Publishers, pp. 563-573.

Tardella, F., 2008. Existence and sum decomposition of vertex polyhedral convex envelopes. Optim. Lett. 2, 363-375.

Tawarmalani, M., Ahmed, S., Sahinidis, N. V., 2002. Product disaggregation in global optimization and relaxations of rational programs. Optim. Eng. 3, 281303.

Tawarmalani, M., Richard, J.-P. P., Xiong, C., 2013. Explicit convex and concave envelopes through polyhedral subdivisions. Math. Program. 138 (1-2), 531577.

Tawarmalani, M., Sahinidis, N. V., 2001. Semidefinite relaxations of fractional programs via novel convexification techniques. J. Glob. Optim. 20, 133-154.

Tawarmalani, M., Sahinidis, N. V., 2002a. Convex extensions and envelopes of lower semi-continuous functions. Math. Program. 93 (2), 247-263.

Tawarmalani, M., Sahinidis, N. V., 2002b. Convexification and Global Optimization in Continuous and Mixed-Integer Nonlinear Programming: Theory, Applications, Software, and Applications. Nonconvex Optimization and Its Applications. Kluwer Academic Publishers, Norwell, MA, USA.

Tawarmalani, M., Sahinidis, N. V., 2004. Global optimization of mixed-integer nonlinear programs: A theoretical and computational study. Math. Program. 99, 563-591.

Tawarmalani, M., Sahinidis, N. V., 2005. A polyhedral branch-and-cut approach to global optimization. Math. Program. 103, 225-249.

Teles, J., Castro, P. M., Novais, A. Q., 2008. LP-based solution strategies for the optimal design of industrial water networks with multiple contaminants. Chem. Eng. Sci. 63 (2), 376 - 394.

Teles, J. P., Castro, P. M., Matos, H. A., 2012. Global optimization of water networks design using multiparametric disaggregation. Comput. Chem. Eng. 40, $132-147$.

Tsoukalas, A., Mitsos, A., 2014. Multivariate McCormick relaxations. J. Glob. 
Vandenbussche, D., Nemhauser, G. L., 2005a. A branch-and-cut algorithm for nonconvex quadratic programs with box constraints. Math. Program. 102 (3), $559-575$.

Vandenbussche, D., Nemhauser, G. L., 2005b. A polyhedral study of nonconvex quadratic programs with box constraints. Math. Program. 102 (3), 531-557.

Vaz, A. I. F., Vicente, L. N., 2007. A particle swarm pattern search method for bound constrained global optimization. J. Glob. Optim. 39 (2), 197-219.

Vaz, A. I. F., Vicente, L. N., 2009. Pswarm: a hybrid solver for linearly constrained global derivative-free optimization. Optim. Method. Softw. 24 (4-5), 669-685.

Viana, F. A. C., Haftka, R. T., Watson, L. T., 2013. Efficient global optimization algorithm assisted by multiple surrogate techniques. J. Glob. Optim. 56 (2), 669-689.

Vicente, L. N., 2009. Implicitly and densely discrete black-box optimization problems. Optim. Lett. 3 (3), 475-482.

Vicente, L. N., Custódio, A. L., 2012. Analysis of direct searches for discontinuous functions. Mathematical Programming 133 (1-2), 299-325.

Vigerske, S., 2012. Decomposition in multistage stochastic programming and a constraint integer programming approach to mixed-integer nonlinear programming. PhD in Mathematics, Humboldt-University Berlin.

Villemonteix, J., Vazquez, E., Sidorkiewicz, M., Walter, E., 2009a. Global optimization of expensive-to-evaluate functions: an empirical comparison of two sampling criteria. J. Glob. Optim. 43 (2), 373-389.

Villemonteix, J., Vazquez, E., Walter, E., 2009b. An informational approach to the global optimization of expensive-to-evaluate functions. J. Glob. Optim. 44 (4), 509-534.

Visweswaran, V., 2009. MINLP: Applications in blending and pooling. In: Floudas, C. A., Pardalos, P. M. (Eds.), Encyclopedia of Optimization, 2nd Edition. Springer Science, pp. $2114-2121$.

Visweswaran, V., Floudas, C. A., 1990. A global optimization algorithm (GOP) for certain classes of nonconvex NLPs: II. application of theory and test problems. Comput. Chem. Eng. 14 (12), 1419 - 1434.

Waki, H., Kim, S., Kojima, M., Muramatsu, M., 2006. Sums of squares and semidefinite program relaxations for polynomial optimization problems with structured sparsity. SIAM J. Optim. 17 (1), 218-242. 
Wan, X. T., Pekny, J. F., Reklaitis, G. V., 2005. Simulation-based optimization with surrogate models - application to supply chain management. Comput. Chem. Eng. 29 (6), 1317-1328.

Wang, B., Gebreslassie, B. H., You, F., 2013. Sustainable design and synthesis of hydrocarbon biorefinery via gasification pathway: Integrated life cycle assessment and technoeconomic analysis with multiobjective superstructure optimization. Comput. Chem. Eng. 52, 55 - 76.

Wechsung, A., Barton, P. I., 2014. Global optimization of bounded factorable functions with discontinuities. J. Glob. Optim. 58 (1), 1-30.

Wechsung, A., Schaber, S. D., Barton, P. I., 2014. The cluster problem revisited. J. Glob. Optim. 58 (3), 429-438.

Westerlund, T., Lundell, A., Westerlund, J., 2011. Some notes on convex relaxations. AIDIC Conference Series 10, 383-392, dOI: 10.3303/ACOS1110042.

Westerlund, T., Skrifvars, H., Harjunkoski, I., Pörn, R., 1998. An extended cutting plane method for a class of non-convex MINLP problems. Comput. Chem. Eng. 22 (3), $357-365$.

Wicaksono, D. S., Karimi, I. A., 2008. Piecewise MILP under-and overestimators for global optimization of bilinear programs. AIChE J. 54 (4), 991 - 1008.

Williams, C. M., Ghobeity, A., Pak, A. J., Mitsos, A., 2012. Simultaneous optimization of size and short-term operation for an RO plant. Desalination 301, 42 -52 .

Wolfe, M. A., 2000. Interval mathematics, algebraic equations and optimization. J. Comput. Appl. Math. 124 (1-2), 263-280.

Yao, W., Chen, X. Q., Huang, Y. Y., van Tooren, M., 2014. A surrogate-based optimization method with RBF neural network enhanced by linear interpolation and hybrid infill strategy. Optim. Method. Softw. 29 (2), 406-429.

Yee, T. F., Grossmann, I. E., 1990. Simultaneous optimization models for heat integrationII. Heat exchanger network synthesis. Comput. Chem. Eng. 14 (10), 1165 - 1184 .

You, F., Grossmann, I. E., 2008. Mixed-integer nonlinear programming models and algorithms for large-scale supply chain design with stochastic inventory management. Ind. Eng. Chem. Res. 47 (20), 7802-7817.

You, F., Grossmann, I. E., 2009a. Mixed-integer nonlinear programming models and algorithms for supply chain design with stochastic inventory management. Available from CyberInfrastructure for MINLP [A collaboration of CMU and IBM Research] at: 
wWw.minlp.org/library/problem/index.php? $i=30$.

You, F., Grossmann, I. E., 2009b. Mixed-integer nonlinear programming models for the optimal design of multi-product batch plant. Available from CyberInfrastructure for MINLP [A collaboration of CMU and IBM Research] at: www.minlp.org/library/problem/index.php? $i=48$.

You, F., Grossmann, I. E., 2010. Integrated multi-echelon supply chain design with inventories under uncertainty: MINLP models, computational strategies. AIChE J. 56 (2), 419-440.

Yuan, Z., Chen, B., 2012. Process synthesis for addressing the sustainable energy systems and environmental issues. AIChE J. 58 (11), 3370-3389.

Zabinsky, Z. B., 2003. Stochastic adaptive search for global optimization. NonConvex Optimization and its Applications. Kluwer, Norwel,MA.

Zhao, Q., Karisch, S. E., Rendl, F., Wolkowicz, H., 1998. Semidefinite programming relaxations for the quadratic assignment problem. J. Combin. Optim. 2, 71-109.

Zhao, Z., Meza, J. C., Hove, M. V., 2006. Using pattern search methods for surface structure determination of nanomaterials. J. Phys. Condens. Matter 18 (39), 8693-8706.

Zondervan, E., Grossmann, I. E., 2009. A deterministic security constrained unit commitment model. Available from CyberInfrastructure for MINLP [A collaboration of CMU and IBM Research] at: www.minlp.org/library/problem/index.php? $i=41$.

Zorn, K., Sahinidis, N. V., 2013. Computational experience with applications of bilinear cutting planes. Ind. Eng. Chem. Res. 52 (22), 7514-7525.

Zorn, K., Sahinidis, N. V., 2014a. Global optimization of general non-convex problems with intermediate bilinear substructures. Optim. Method Softw. 29 (3), 442-462.

Zorn, K., Sahinidis, N. V., 2014b. Global optimization of general nonconvex problems with intermediate polynomial substructures. J. Glob. Optim. 59 (23), 673-693. 